\title{
KSHV Episome Tethering Sites on Host Chromosomes: Regulation of Latency-Lytic Switch by the ChAHP complex
}

\section{Ashish Kumar}

UC Davis School of Medicine

Yuanzhi Lyu

UC Davis School of Medicine

Yuichi Yanagihashi

Lifematics

Chanikarn Chantarasrivong

Lifematics

\section{Vladimir Majerciak}

National Cancer Institute https://orcid.org/0000-0003-2907-4583

\section{Michelle Salemi}

University of California, Davis

\section{Kang-Hsin Wang}

UC Davis

Frank Chuang

UC Davis

Ryan Davis

UC Davis https://orcid.org/0000-0001-7439-6683

Clifford Tepper

UC Davis https://orcid.org/0000-0001-7105-1102

Kazushi Nakano

Lifematics

Chie Izumiya

UC Davis

Michiko Shimoda

UC Davis

Ken-ichi Nakajima

UC Davis

Alexander Merleev

UC Davis

Zhi-Ming Zheng 
Tumor Virus RNA Biology Section, HIV Dynamics and Replication Program, Center for Cancer Research, National Cancer Institute, National Institutes of Health, Frederick, MD 21702, USA

Mel Campbell

UC Davis https://orcid.org/0000-0002-8475-6686

Yoshihiro Izumiya ( $\square$ YIZUMIYA@UCDAVIS.EDU)

UC Davis School of Medicine https://orcid.org/0000-0002-9184-2603

\section{Article}

Keywords: LANA, ChAHP, KSHV, Episome Tethering Sites

Posted Date: September 22nd, 2021

DOI: https://doi.org/10.21203/rs.3.rs-136834/v2

License: (c) (i) This work is licensed under a Creative Commons Attribution 4.0 International License. Read Full License 
Word counts:

* Correspondence: Mel Campbell, PhD

Address: UCDMC Research III, Room 3300D, $46452^{\text {nd }}$ Avenue, Sacramento CA 95817

E. mail: mcampbell@ucdavis.edu

Phone: 916-734-7811

*Correspondence: Yoshihiro Izumiya DVM, PhD

Address: UCDMC Research III, Room 2200B, $46452^{\text {nd }}$ Avenue, Sacramento CA 95817

E. mail: yizumiya@ucdavis.edu

Phone: 916-734-7253 


\section{Abstract}

40 Kaposi's sarcoma-associated herpesvirus (KSHV) establishes a latent infection in the cell

41 nucleus, but where KSHV episomal genomes are tethered and the mechanisms underlying

$42 \mathrm{KSHV}$ lytic reactivation are unclear. Here, we study the nuclear microenvironment of KSHV

43 episomes and show that the KSHV latency-lytic replication switch is regulated via viral long non-

44 coding (Inc)RNA-CHD4 (chromodomain helicase DNA binding protein 4) interaction. KSHV

45 episomes localize with a CHD4 complex, ChAHP, at epigenetically active genomic regions and

46 tethers frequently near centromeric regions of host chromosomes. The ChAHP complex also

47 occupies the 5'-region of a highly-inducible IncRNAs and terminal repeats of KSHV genome with

48 latency-associated nuclear antigen (LANA). Viral IncRNA binding competes with CHD4 DNA

49 binding, and KSHV reactivation is accompanied by the detachment of KSHV episomes from host

50 chromosome docking sites We propose a model in which elevated IncRNA expression

51 determines the KSHV latency-lytic decision by regulating LANA/ChAHP DNA binding at 52 inducible viral enhancers. 


\section{Introduction}

Kaposi's sarcoma-associated herpesvirus (KSHV), discovered in 1994, is one of eight human herpesviruses (1). Since then, KSHV has been identified as the causative agent of Kaposi's sarcoma (1-3) and two human lymphoproliferative diseases, primary effusion lymphoma (PEL) and AIDS-related multicentric Castleman's disease (4-7). Although HAART (highly active antiretroviral therapy) of HIV-patients dramatically reduced KS incidence in Western countries, KSHV associated-malignancies are still a leading cause of cancer deaths in AIDS patients in Sub-Saharan Africa. Additional therapeutic measures are urgently needed. In cancer cells, KSHV establishes a latent infection in which most viral genes are silenced with the exception of few latent proteins, such as latency associated nuclear antigen (LANA) (8). External stimuli trigger KSHV reactivation in latently infected cells through activation of highly potent viral transactivator, K-Rta, which then activates early gene expression including three viral long noncoding RNAs (9-11).

KSHV LANA is a $130 \mathrm{kDa}$ multifunctional protein, which plays a major role in both DNA replication and episome maintenance during latency. LANA binds DNA sequences within the 801 base pair terminal repeats (TRs) of the KSHV genome, which serve as both an origin of DNA replication and a site for tethering episomes to the host cell chromosomes (12-15). There are two distinct mechanisms through which LANA tethers episomes to host cell chromosomes. First, the N-terminus of LANA, which is predicted to be highly-unstructured, can interact with host chromosomes through direct binding with histone H2A and H2B $(16,17)$. A second mechanism involves the interaction of the LANA DNA binding domain (DBD) with host chromatin-associated proteins (18-20). The chromatin-associated proteins include BRD2 and BRD4, which contain two bromodomains that recognize the acetylated $\mathrm{H} 3$ and $\mathrm{H} 4$ histones, and 
77 a conserved C-terminal extraterminal domain $(21,22)$. The BRD extraterminal domain interacts directly with the LANA DBD, providing a putative mechanism for episome docking sites selection at epigenetically active host chromosomes $(18,23)$. In both mechanisms, tethering requires a sequence-specific interaction between the LANA DBD and the viral episome at LANA binding sites 1 and 2 (LBS 1/2) (15). Episome maintenance requires at least two copies of LBS1/2 and the KSHV genome encodes 30 to 40 terminal repeats, taking up to approximately one fifth of KSHV genome (24-32 kbp DNA fragment) (24). The crystal structures of the LANA DBD further revealed that LANA can form a higher-order decameric ring structure, and a hydrophobic interface between LANA dimers to form the decameric ring is important for cooperative LBS $1 / 2$ binding, DNA replication, and thereof episome maintenance (25). The crystal structure of LANA DBD also reveals distinct binding interfaces among BRD2/4 binding site, DNA binding, and decameric interfaces $(25,26)$.

Spatial and temporal organization of the genome plays a critical role in gene expression in many organisms including large DNA viruses $(27,28)$. Similar to cellular genes, the KSHV episome changes its genomic structure with increasing genomic looping at $\underline{K} S H V \underline{\text { Replication }}$ and Transactivation (K-Rta) binding sites during KSHV reactivation (28). Imaging studies suggested that DNA/RNA/protein aggregates are formed at actively transcribed sites, in which a significant fraction of RNA Polymerase II (RNAPII) is recruited to the KSHV genomes and used to transcribe viral genomes during KSHV reactivation (29). Such transcription factory formation is known to bring gene promoters and enhancers together (27). It is clear that dynamic regulation of genome architecture plays a critical role in gene transcription, hence outcomes of KSHV latency-lytic replication decisions. However, the mechanisms that control KSHV episome architecture remains elusive. 
Chromatin remodeling enzymes have been of wide interest in the regulation of KSHV 101 latency-lytic switch. The enzymes include polycomb repressor complex 1 and 2, nucleosome remodeling and deacetylase (NuRD), lysine demethylases (KDM2B, 3A, and 4A), histone lysine acetylase and methylases (20, 30-35). Recent studies highlight that chromatin remodeling enzymes also participate in regulation of genome architecture. In murine embryonic stem cells, 105 the Iswi family remodeler Snf2h promotes CTCF binding to the genome and regulates formation 106 of contact domains $(36,37)$. In addition to Snf2h, CHD4 (chromo domain helicase DNA binding 107 protein 4) has recently been shown to occupy and restrict enhancer accessibility as well as 108 cohesin occupancy at CHD4 binding sites (38). Genetic disruption of CHD4 therefore causes spontaneous differentiation concomitant with premature activation of lineage-specific genes (3941). CHD4 has been identified in two distinct cellular protein complexes, the NuRD and ChAHP 111 complexes (42). The ChAHP complex consists of CHD4, ADNP (activity-dependent 112 neuroprotective protein), and $\mathrm{HP} 1 \gamma$ (heterochromatin protein 1) (39). ANDP binds DNA sequence motifs such as GCCCTCTTCTGG and anchors the ChAHP complex to euchromatin (43). ChAHP complex was shown to modulate spatial chromatin loop organization locally, and counteract chromatin looping at CTCF sites (43). Accordingly, ChAHP plays essential roles in maintaining distinct cellular states and ensure accurate cell fate decisions in response to external signals during development that are likely to associate with its function in regulation of enhancer accessibility $(39,41,44)$. Because of CHD4's roles in enhancer regulation, it is not surprising that mutation or overexpression of CHD4 has been linked to multiple developmental disorders and malignancies in humans (45-48).

The long non-coding (Inc)RNAs that are transcribed from active enhancers in a cell- or

122 tissue- specific manner are called enhancer RNAs (eRNAs) (49). The functions of eRNAs are 
123 largely mediated through interactions with eRNA binding partners (50-52); however, studies also 124 indicated that RNA sequence is not contributing to the specificity of eRNA-protein interactions. 125 Instead, specificity of the function is suggested to be achieved through gene/RNA positioning 126 and spatial proximity to regulated genomic regions (53). Similarly, the KSHV genome encodes 127 at least three highly inducible IncRNAs (Ori-RNA [also called T1.5], ㅇoly Adenylated Nuclear 128 RNA [PAN RNA], and T0.7) that are transcribed from epigenetically active regions, and are direct 129 targets of K-Rta (11). The PAN RNA genomic locus actively recruits cellular RNAPII during 130 KSHV reactivation, leading to significantly higher copy number of PAN RNA molecules (up to $13110^{5}$ copies) in a reactivating cell (54). The high copy number of PAN RNA has also been 132 explained by its higher RNA stability (55). Three sequence elements, ENE (expression and 133 nuclear retention element) at the $3^{\prime}$ region, MRE (Mta responsive element) at the $5^{\prime}$ region of 134 PAN RNA, and the structure of the poly (A) tail, are critical for PAN RNA stability (56-58). PAN 135 RNA interacts with multiple cellular and viral proteins $(57,59)$, and functions as a transactivator 136 by sequestering repressors and/or recruiting histone modifying enzymes (32, 60, 61). A recent 137 report shows that PAN RNA could be replaced by other viral IncRNA sequences without a 138 significant loss of KSHV replication, suggesting that there is a sequence-independent function 139 similar to that of eRNAs (62).

In this study, we provide evidence that the KSHV latent-lytic switch is regulated by the 141 ChAHP complex. We mapped KSHV episome tethering sites on host chromosomes with 142 Capture Hi-C and studied the surrounding nuclear microenvironment of KSHV episomes using 143 proximity biotin labeling. We show that KSHV episomes co-localize with the ChAHP complex at 144 epigenetically active host chromosomes, and CHD4 interaction with viral IncRNAs regulates 145 binding of CHD4 on KSHV chromosomes, which de-represses viral IncRNAs from ChAHP- 
146 mediated repression. We propose that the inducible enhancer activity is regulated by a balance

147 between local eRNA transcription activity and ChAHP complex tethering, and the mechanism 148 regulates $\mathrm{KSHV}$ reactivation.

\section{Results}

\section{Identification of KSHV episome tethering site on host chromosomes.}

$152 \mathrm{KSHV}$ episomes tether to the host cell chromosomes via LANA, however the mechanism of 153 selection of docking sites is not very well characterized. To understand how and where KSHV 154 episomes tether to host chromosomes, we applied $\underline{\text { Capture }} \underline{\mathrm{Hi}-\mathrm{C}}(\mathrm{CHi}-\mathrm{C})$ method to identify 155 episome docking sites in three KSHV naturally-infected PEL cell lines, BC-1, BC-3 and BCBL156 1. The schematic diagram for the $\mathrm{CHi}-\mathrm{C}$ procedure is presented in Fig. 1a. In order to obtain 157 high resolution, the $\mathrm{Hi}-\mathrm{C}$ sequencing library was further enriched by using biotinylated tiling-oligo 158 probes that specifically hybridized to KSHV DNA sequences (Fig. 1a). Using this method, we 159 examined the position of host genomic regions that exhibited higher frequencies of normalized 160 chimeric ligation reads with KSHV DNA fragments. We identified selectively enriched chimeric 161 ligation reads throughout the host chromosomes (Fig. 1b). The mapped reads on 23 individual 162 chromosomes for BC-1, BC-3 and BCBL-1 are presented separately in Supplementary Fig. 1a163 c. Each dot represents the number of normalized contact heterotypic sequence reads between 164 host and KSHV sequences. The $\mathrm{CHi}-\mathrm{C}$ normalized chimeric read counts on chromosome 1 for 165 three naturally infected PEL cells are shown in Fig. 1c. The results indicated that there are a 166 higher number of chimeric read counts near centromeric regions in all three cell lines (Fig. 1c). 167 To study the frequencies of chimeric read counts that are located near the centromere region, 
168 we performed a mathematic characterization, in which we extracted the chimeric read counts 169 that derived from regions spanning a distance corresponding to $1 \%$ of the size of each 170 chromosome at either 5 ' or $3^{\prime}$ centromere chromosome regions (marked green and blue). Counts 171 per $100 \mathrm{kbp}$ were calculated separately for each chromosome and compared to the average 172 number across the individual chromosome. The results showed that higher chimeric read counts 173 were seen ( 15 out of 23 chromosomes in $\mathrm{BCBL}-1$ ) either $5^{\prime}$ or $3^{\prime}$ regions of centromere, and in 174 some instances (e.g., chromosome 8, 19, X), we observed enrichment in both 5' and 3' positions 175 (Fig. 1d). To further confirm the $\mathrm{CHi}-\mathrm{C}$ findings, we performed DNA-FISH with centromere176 specific PNA (peptide nucleic acid) probes in combination with LANA immunostaining. These results further showed that a large fraction of LANA dots was colocalized with centromeres (Fig. 1e, Supplementary Fig. 2). The results are consistent with a previous report, which demonstrated that LANA colocalized with centromeric protein F (CENPF) and kinetochore 180 protein, Bub1, and observed at centromere regions during metaphase (63). To determine whether KSHV docking sites are random, we next examined the similarity of KSHV episome tethering sites among the three cell lines using a Jaccard Index. We calculated the similarity of tethering sites based on the positions of chimeric sequence reads. The index identified $97.86 \%$ (BC-1 vs BC-3), $81.99 \%$ (BC-1 vs BCBL-1) and $82.36 \%$ (BC-3 vs BCBL-1) similarity (Fig. 1f).

185 The results demonstrated that a majority of KSHV episomes tethers the similar host genomic 186 regions in three naturally infected PEL cell lines, suggesting that there is a preferential nuclear 187 microenvironment that can attract/maintain KSHV latent episomes during cell divisions. 
191 Next, we examined the nuclear protein microenvironment of KSHV episome-tethering sites in 192 infected cells. We hypothesized that, by examining cellular proteins neighboring to LANA in 193 infected cells, we should be able to identify the repertoire of proteins important for tethering and 194 selection of KSHV episome-docking sites. To identify proteins in close proximity to LANA, we 195 used a miniTurbolD based method. The miniTurbolD is a biotin ligase, which covalently attaches 196 biotin to lysine residues in neighboring proteins $(<10 \mathrm{~nm})$ in less than 10 minutes with no 197 significant cell toxicity $(64,65)$. A recombinant KSHV BAC16 with LANA N-terminal tagged with 198 miniTurbolD (referred to as KSHV LANA-mTID) was prepared; the procedure for the preparation 199 of KSHV LANA-mTID is presented in Supplementary Fig. 3. We transfected the iSLK cells with 200 the recombinant KSHV LANA-mTID and stably selected with hygromycin ( $1 \mathrm{mg} / \mathrm{ml})$. The KSHV 201 LANA-mTID virus was then recovered by inducing reactivation with doxycycline $(1 \mu \mathrm{g} / \mathrm{ml})$ and 202 sodium butyrate $(3 \mathrm{mM})$ for 5 days. iSLK cell line was infected with recombinant KSHV LANA$203 \mathrm{mTID}$ virus and selected with hygromycin $(1 \mathrm{mg} / \mathrm{ml})$ to generate stable iSLK-LANA mTID cells 204 (Fig. 2a). The iSLK-LANA mTID cells were incubated with D-biotin for 60 minutes in culture 205 media and biological triplicated samples were prepared (Fig. 2b). This strategy led us to identify 20676 host proteins $(P<0.05)$ that were physically neighboring KSHV LANA within $10 \mathrm{~nm}$ radial 207 distance during the period of D-biotin incubation (64). The 76-host proteins include nuclear 208 mitotic apparatus protein (NuMA), bromodomain-containing protein 4 (BRD4), and lysine209 specific demethylase 3A and 3B (KDM3A and 3B) that have been previously shown to physically 210 interact with LANA (20, 66-68) (Fig. 2c, Supplementary Table 1). In addition to those previously 211 identified cellular proteins, the study also precipitated components of the ChAHP complex (39), 212 which is composed of chromodomain helicase DNA-binding protein 4 ( 213 dependent neuroprotector homeobox protein ( $\underline{A D N P})$ and $\underline{\mathrm{HP}}-1 \mathrm{\gamma}$ with high confidence (Fig. 2c). 
Although we could not identify HP-1Y with our statistical criterion in proteomics study, HP-1Y protein was previously shown to interact with KSHV LANA (69). The LANA interaction with CHD4 and ADNP was further validated with in vitro pull-down assays, and the results showed that LANA could interact with CHD4 and ADNP in the absence of other viral proteins (Fig. 2d). Further, recombinant GST-tagged LANA deletion proteins (Supplementary Fig. 4) were used to map the interaction domain with CHD4 and found that the amino acid (aa) residues 870-1070, near the LANA DNA-binding domain, were responsible for interaction with CHD4 (Fig. 2e). Immunofluorescence assays with mono-specific antibodies further confirmed that LANA and CHD4 were colocalized in naturally infected BCBL-1 cells (Fig. 2f). Taken together, these results suggest that LANA is able to associate with the ChAHP complex in latently infected cells.

\section{Association of KSHV episome-tethering sites with ChAHP complex binding.}

The protein interaction and colocalization between CHD4 and LANA "dots" in the nucleus led us to further investigate the localization of CHD4, ADNP and LANA on both host and KSHV chromosomes. To identify the chromatin occupancy site(s) for ChAHP complex and LANA, we employed Cleavage Under Targets and Release Using Nuclease (CUT\&RUN) (70). The LANA, CHD4, and ADNP CUT\&RUN peaks clearly overlapped at multiple sites of cell host chromosomes with active histone marks (H3K27Ac), which include previously described IRF4 super enhancer region $(71,72)$ (Fig. 3a). NGS plots between LANA CUT\&RUN summit peaks, and CHD4 or ADNP further confirmed co-occupancies of LANA and the ChAHP complex on the host chromosomes (Fig. 3b). Because LANA and ChAHP complex interact with each other, we hypothesized that LANA-ChAHP complex could be important for tethering KSHV episomes to host chromosomes. To test this, we generated NGS plots between LANA, CHD4 and ADNP 
237 CUT\&RUN summit peaks, and distribution of $\mathrm{CHi}-\mathrm{C}$ chimeric reads. We observed that KSHV 238 episome tethering sites were indeed primarily localized near the LANA, CHD4 and ADNP binding 239 sites, whereas it was not observed for H3K4me1 (Fig. 3c) or H3K27me3 (data not shown). To 240 further calculate degree of interaction mathematically, we measured the relative $\mathrm{CHi}-\mathrm{C}$ chimeric 241 reads per million and examined association with relative distance with CHD4 binding site. 242 Accumulation index calculated from NGS plot suggested that more than $50 \%$ of $\mathrm{CHi}-\mathrm{C}$ reads are 243 closely located at CHD4 binding site within the genomic regions (Fig. 3d). Altogether, these results suggest that KSHV episome tethers to host chromosome at the ChAHP binding sites.

KSHV LANA colocalization with ChAHP complex on KSHV genome.

We next examined ChAHP binding sites on the KSHV episome. Consistent with studies with cellular chromosomes, CHD4 and ADNP occupy genomic loci with active histone marks, which includes KSHV long non-coding RNAs (PAN RNA, T0.7, and T1.5 [Ori-RNA]) promoter regions (Fig. 4a). The results also showed colocalization among LANA, CHD4, and ADNP with the active histone mark, H3K27Ac, along the KSHV genome, and exceptionally strong peaks were seen at terminal repeat regions (read counts are depicted in Fig. 4a), where multiple copies of LANA bind (73). The strong peaks at TR regions are likely due to a combination of tighter binding of the complex and the presence of multiple copies of the same sequences. The strong signals at TR regions are unlikely due to mapping problems, because H3K27me3 showed a lower number of sequence reads compared with sequence reads in the unique region. The three viral IncRNAs, especially PAN RNA, are known to be expressed at significantly higher transcript copy numbers than the open reading frames during lytic replication $(54,74)$, and CHD4, ADNP, and LANA were clearly localized at the 5 ' regions of these IncRNA promoter regions (Fig. 4a). Next, the effects 
of KSHV reactivation on CHD4 occupancies were examined by CUT\&RUN with qPCR. The results suggested that $\mathrm{CHD} 4$ occupancies on the KSHV genome were reduced during KSHV reactivation (Fig. 4b). We further studied effects of KSHV reactivation on the KSHV episome tethering with the ChAHP complex on host cell chromosomes. For this, TREx-BCBL-1 cells were reactivated for twenty-four hours and $\mathrm{CHi}-\mathrm{C}$ samples were prepared. We measured the relative chimeric DNA sequence frequencies of KSHV with host chromosomes before and after KSHV reactivation. The relative amount of chimeric sequence reads at one of the KSHV episome tethering sites were visualized with Juicebox. By subtracting the number of relative sequence reads in latent samples from those in reactivated samples, we also visualized changes by induction of active viral transcription. The results showed that KSHV chimeric sequence reads were reduced, suggesting that KSHV reactivation induced detachment of the KSHV episome from host chromosomes, and a similar detachment was also seen in other putative episometethering sites (Fig. 4c). The results suggest that robust viral IncRNAs at the ChAHP binding site may play a role in detachment of ChAHP/LANA complex from chromosomes.

\section{Identification of PAN RNA binding proteins with proximity biotin ligation.}

Previous studies including ours suggested that PAN RNA transcription in cis plays an essential role in initiation of KSHV lytic replication. Inhibition of PAN RNA transcription by either deletion of the PAN RNA sequence or mutation at K-Rta responsive elements in the PAN RNA promoter significantly impaired $\mathrm{KSHV}$ reactivation and replication $(28,60)$. In addition, CHD4 is known to bind cellular enhancers to regulate enhancer-promoter interactions, and enhancers often transcribed enhancer RNAs (IncRNAs) (75). To examine PAN RNA-mediated transcription regulation, we again applied the proximity labeling technique to profile PAN RNA neighboring 
283 proteins during KSHV reactivation. This time, the mTID cassette was first inserted as an N284 terminal fusion of ORF57, a PAN RNA binding protein, and then the fusion protein was expressed from the endogenous promoter during KSHV reactivation (Fig. 5a). Taking advantage of a previous detailed mapping study, which identified ORF57 binding sites on PAN RNA, termed MRE (Mta Responsive Element) (57), we utilized a PAN MRE mutant virus having a 9-nucleotide mutation in the MRE element (Fig. 5a). KSHV genome-wide qRT-PCR array analysis confirmed previous studies that the PAN RNA MRE mutation impaired viral gene expression, with stronger effects being seen in late gene cluster regions (Supplementary Fig. 5a right panel). We also 291 confirmed that protein biotinylation happened only in the presence of both ORF57 protein (reactivated sample) and biotin (Fig. 5b). By comparing the enrichment profiling between the PAN RNA MRE mutant and PAN RNA Wt, we isolated cellular proteins that are in proximity to proteins from mTID-57 PAN Wt iSLK cells and mTID-57 PAN-MRE iSLK cells, respectively $(p<0.05)$ (Supplementary Table 2). Among the interacting proteins, 74 proteins were common between the PAN MRE Wt and PAN MRE mutant, while 55 proteins were found only in the presence of the wild type PAN RNA sequence (Fig. 5c, d). Deletion of MRE seemed to unleash ORF57 protein and allow ORF57 to interact more freely with other RNA binding proteins (Fig. 5c). Importantly, this proteomics approach identified CHD4 as a putative PAN RNA binding protein $(p<0.005)$. Next, we performed the siRNA screening of $129(55+74)$ PAN RNA binding proteins with KSHV reactivation indicator cell line, iSLK.219. The knockdown screening identified 4 strong repressors namely IK, CHD4, PABPC3 and RPL18A with fold change (RFP/GFP) $>3$ among the 129 proteins, and CHD4 was found be the strongest among them (RFP/GFP > 7fold) (Fig. 5e). Gene Ontology (GO) analysis of 129 PAN RNA-mediated ORF57-interacting 
proteins suggested that ORF57 is indeed primarily involved in RNA processing (Supplementary Fig. 5b).

\section{A viral IncRNA inhibits CHD4 dsDNA binding.}

PAN RNA MRE-dependent enrichment by ORF57-mediated biotinylation suggested that CHD4 is an RNA-binding protein, and that PAN RNA brings the CHD4 and ORF57 proteins into proximity by serving as a scaffold for the biotinylation (model shown in Fig. 5a). In order to examine the interaction of CHD4 with PAN RNA, we prepared purified RNAs (PAN RNA, MRE mutant, PAN RNA deletion mutants and luciferase RNA) and proteins (CHD4, NF-KB and Luciferase) (Fig. 5f) and performed in vitro interaction assays (depicted in Fig. 5g). The results showed that CHD4 was indeed precipitated with PAN RNA; however, MRE mutant, PAN RNA deletion mutants, as well as irrelevant luciferase RNA also interacted with CHD4 protein, suggesting that CHD4 RNA binding is unlikely sequence specific under our binding conditions (Fig. 5h). However, the same RNA binding conditions with full length PAN RNA did not precipitate a DNA binding protein, NF-kB (p65) or Luciferase protein, suggesting that CHD4 does possess RNA-binding capacity (Fig. 5i). Because a previous study showed that Drosophila melanogaster CHD4 homolog is capable of binding DNA and human CHD4 ATPase activity is stimulated in the presence of naked DNA (76), we tested if purified CHD4 protein is able to bind to double stranded (ds)DNA fragment encoding the PAN RNA sequence. Biotinylated primer was used to amplify DNA fragment from the PAN RNA gene and used for biotin-pull down analyses. The results showed that CHD4 was able to bind dsDNA directly (Fig. 5j). Importantly, increasing amounts of PAN RNA (ssRNA) antagonized CHD4 dsDNA binding, which was completely blocked in presence of non-biotinylated PAN RNA at 1:10 (dsDNA/RNA) molecular 
329 ratio (Fig. 5j). The results suggested that locally transcribing long non-coding RNAs (which would reach up to $3 \times 10^{5}$ copies/cell for PAN RNA (54)) may remove CHD4 locally from the KSHV genome, as seen by reduced CHD4 on KSHV genome (Fig. 4b) and detachment of KSHV episomes from host chromosomes during reactivation (Fig. 4c). These results suggest that amount of long non-coding RNAs expression near the CHD4 binding sites may play a role in both lytic gene induction and episome tethering.

\section{CHD4 is important for latency maintenance and establishment.}

The studies above suggest that LANA interacts with the ChAHP complex on both cellular and viral chromosomes, and that the ChAHP complex may restrict KSHV enhancer activity and hence KSHV lytic reactivation. Accordingly, we next examined the significance of CHD4 in the KSHV transcription program. CHD4 expression in iSLK.219 cell was knocked down with shRNA and the degree of KSHV reactivation was examined after triggering K-Rta expression with doxycycline. The results showed that knock-down of CHD4 enhanced KSHV replication more expression of mouse Chd4 cDNA (i.e., in order to escape from the shRNA, which targets human CHD4) counteracted effects of CHD4 knock-down (Fig. 6a). Notably, over expression of mouse Chd4 almost completely abolished K-Rta mediated KSHV reactivation (Fig. 6a, second bar), and inhibited the aggregation of RNAPII on the KSHV genome, which was measured by immunostaining with overexpressed CHD4 and RNAPII (Fig. 6b). Strong silencing effects were CHD4's ATPase-activity dependent, because mutations in the helicase domain, using the same mutation found in patients with CHD4-associated syndrome $(42,77)$, was found to increase 351 RNAPII aggregation and KSHV transcription (Fig. 6b, c). In addition to the knock-down or over 
352 expression studies, we also performed single cell transcriptomic studies with reactivated 353 iSLK.219 cells. The results clearly indicated that the presence of higher levels of CHD4 had clear 354 inhibitory effects for the triggering and/or prolonging lytic viral gene transcription burst (Fig. 6d). 355 Further, KSHV transcripts were extracted and sorted based on sequence counts and examined 356 for correlation with cellular gene expression at the single cell level (Supplementary Fig. 6a, b). 357 The results again showed a negative correlation between CHD4 expression and the amount of $358 \mathrm{KSHV}$ transcripts in the cell (Fig. 6e), while a similar negative correlation was not observed for 359 GAPDH or similarly expressing HDAC2, a component of NuRD complex (Supplementary Fig. $3606 c)$.

Finally, the significance of CHD4 in the establishment of latency was also examined. To 362 do this, we first knocked-down CHD4 in 293T cells and infected the cells with purified KSHV r.219 virus to monitor viral gene silencing. CHD4 knock-down was confirmed at the protein and 364 RNA levels (Fig. 6f, g). The results showed that KSHV gene expression continued to increase 365 during a 3-day period in two independent CHD4 KD cells, while KSHV gene expression did not 366 increased significantly in shScramble cells at day 3 (Fig. 6h). Further, KSHV lytic replication was 367 also monitored with RFP signals within the cell population, and results showed that the number 368 of RFP positive cells within the dish were higher in CHD4 knock-down 293T cells compared to 369 negative control knock-down ( $\mathrm{siC}$ ) cells at 96 hours post infection (Fig. 6i, Supplementary Fig. 370 7). Altogether, these results suggest that CHD4 functions to silence KSHV lytic genes at an early 371 stage of KSHV de novo infection and facilitate the entry and maintenance of latency, perhaps 372 by suppressing robust viral IncRNA expression for the viral enhancer activity.

\section{Discussion}


Recent exciting studies suggest that an RNA-binding domain in transcription factor CTCF is essential for the formation of subsets of genome architecture $(78,79)$. The presence of distinct classes of RNA-binding protein (RBP)-dependent genomic loop formation suggests the partition of nascent RNAs and other RBP partners in chromatin-looping regulation at transcriptionally active genomic loci. It is also known that eRNAs and RNA-binding proteins participate in transcriptional factories $(75,80,81)$. We show here that overexpression of CHD4 prevents RNAPII aggregate formation and inhibits KSHV reactivation (Fig. 6a,b). Regulation of the KSHV latency-lytic switch by CHD4 chromatin binding and inhibition of CHD4 DNA binding via inducible transcription in cis may necessitate for KSHV to evolutionally maintain the viral nuclear IncRNAs, which are often expressed at levels 1,000x greater than the majority of KSHV protein-coding RNAs during reactivation (54). Evolution of this RNA as a nuclear and non-coding species may also avoid competition for protein translation and RNA export machineries, while functioning to trigger robust enhancer activity for KSHV genome. Consistent with $\mathrm{CHD} 4$ being a strong repressor, loss-of-function mutations induce enhancer activation leakage, which associates with multiple developmental disorders in humans $(44,47,82-85)$. We think that such CHD4 functional loss may result in an increased propensity to form active enhancer-promoter interactions and prolong gene transcription similar to our observations in KSHV-infected CHD4-KD 293T cells (Fig. 6h). Consistent with CHD4 functions in enhancer access restriction, the ChAHP complex is also known to compete with CTCF binding, and counteract chromatin looping at CTCF binding sites. Therefore, ChAHP maintains evolutionarily conserved spatial chromatin organization by preventing new CTCF binding events that emerged through short Interspersed element expansions (43); this ChAHP biological role suggests that KSHV cleverly find/build a "safe basecamp" with ChAHP to maintain viral episomes structure during evolution. 
A study from Dr. Lieberman's group demonstrated that EBV episomes are tethered in the neighborhood of transcriptionally silent neuronal genes (86). Our CHi-C study also found KSHV 400 episomes frequently localize at the junctions between eu- and hetero-chromatin

(Supplementary Fig. 8 - junction of stripes), but more frequently localizes near centromere regions than to other genomic regions. The advantage of tethering at near centromere remains unknown; however, previous studies also showed that LANA dots are localized at centromeres 404 and LANA interact with centromeric protein F and kinetochore protein, Bub1(63); the two 405 406 on host chromatin and interact with LANA at the hydrophobic interface of LANA dimers near the LANA DNA binding domain $(25,26)$. Suppression of KSHV lytic genes by CHD4 in de novo infected cells also suggests that there might be active recruitment of the ChAHP complex to the incoming KSHV genomes. However, the mechanism(s) that enable KSHV episomes to select specific ChAHP binding sites on host chromosomes, and whether these CHD4 occupied enhancers are linked to tissue-specific gene expression therefore KSHV tissue tropism have yet to be characterized. At least in the three PEL cell lines examined, one of the common ChAHP417 LANA complex-recruitment site is IRF4 super enhancer region, which is highly active in the Bcell lineage $(71,72,87)$. It will be important to know if KSHV episome-bound cellular "enhancers" are annexed and subjected to control by KSHV proteins in infected cells. 
KSHV has been evolved to maintain a mysterious IncRNA, PAN RNA, which is expressed

421 at a very high copy number in the presence of ORF57 protein (54), and deletion of PAN RNA or

422 ORF57 significantly impairs the entire viral lytic gene expression program $(28,60,88)$. How PAN

423 RNA expression activates the expression of distantly localized viral open reading frames is an

424 important question. Because PAN RNAs exceeds $10^{5}$ copies, and CHD4 localizes at PAN RNA

425 transcription initiation sites with LANA (Fig. 4a), we expect that the local PAN RNA concentration

426 would easily result in an RNA-DNA ratio in excess of 10:1 in presence of ORF57 (57). Based on

427 frequent genomic looping, non-coding RNA expression, and CHD4 binding (e.g., which 428 frequently targets enhancers), we propose that the IncRNA encoding regions [Ori-RNA (T1.5), 429 PAN RNA, K12 (T0.7)] function as inducible enhancers for expression of the KSHV ORFs, and 430 KSHV reactivation (e.g., promoter activation) hinges on IncRNA-CHD4 interactions for the de431 repression. Similar to CHD4, we previously demonstrated that PAN RNA expression also 432 sequesters LANA from unique regions of KSHV genomes (61). Our CUT\&RUN studies showed 433 that CHD4 and LANA colocalized on both the KSHV and host genomes (Fig. 4a) and they could 434 physically interact with each other (Fig. 2d-f); thus, we favor the idea that reactivation is triggered 435 by detachment of LANA/ChAHP complex-loaded TR fragments from the KSHV unique region 436 (ORFs-encoded region as well as host chromosome) by robust expression of IncRNA, whose 437 expression is directly activated by K-Rta and enhanced by ORF57. In this proposed model, 438 transcriptional burst by a strong viral transactivator, results in the local appearance of multiple 439 nascent and stable-RNA copies simultaneously, in turn increasing local nucleic acid density 440 before PAN RNA is diffused into the nuclear neighborhood. Notably, our KSHV 3D genomic 441 structure modeling based on frequencies of $\mathrm{CHi}-\mathrm{C}$ reads within $\mathrm{KSHV}$ sequences suggested 442 that these highly inducible IncRNA encoding regions localize spatially in close proximity to TRs 
443 in 3D (Model in Fig. 6j, Campbell et al., in preparation). The results combined with this study 444 suggest that the ChAHP-LANA complex has an architectural role for KSHV latent genomic 445 structure, and the TR region that recruits significant number of copies of both ChAHP and LANA 446 (Fig. 4a) is critical for episome structure and therefore suppression of lytic genes epigenetically. 447 Disruption of such a "backbone" by CHD4 KD or by robust expression of PAN RNA which binds 448 CHD4 therefore triggers "leakage" of viral lytic gene expression similarly to premature activation 449 of lineage-specific genes [(39-41) and Fig. $\mathbf{6 g})]$. Structural studies for the ChAHP/LANA protein 450 complex with TR unit and recombinant KSHV with a LANA CHD4 binding mutant would further 451 clarify the significance of the interaction and possibly lead to small molecular drugs to target the 452 interaction.

In summary, we have demonstrated that the ChAHP complex is a key regulator of the KSHV latency-lytic switch. With strong effects of CHD4 on KSHV replication, it will be important 455 to study how ChAHP complexes are regulated in infected cells, and also a relationship with $456 \mathrm{KSHV}$ replication and KSHV-mediated disease progression in individuals who unfortunately 457 possess CHD4 mutations.

\section{Methods}

Materials. Dulbecco's Modified Eagle medium (DMEM), RPMI 1640 medium, fetal bovine serum 461 (FBS), phosphate buffered saline (PBS), Trypsin-EDTA solution, 100x Penicillin-streptomycin462 L-glutamine solution and Streptavidin-HRP conjugate were purchased from Thermo Fisher 463 Scientific (Waltham, MA USA). Puromycin and G418 solution were obtained from InvivoGen 464 (San Diego, CA, USA). Hygromycin B solution was purchased from Enzo Life Science 465 (Farmingdale, NY, USA). Anti-LANA (clone LN53), anti-Flag (clone M2) and anti-RNAPII (clone 
CTD4H8) antibodies were purchased from Millipore-Sigma. Anti-CHD4 (D4B7), H3K27Ac (D5E4) and H3K4me1 (D1A9) antibodies were purchased from Cell Signaling Technology. AntiADNP (PA5-52286) antibody was purchased from Invitrogen. Streptavidin magnetic beads (Pierce) were purchased from Thermo Fisher Scientific ${ }^{\mathrm{TM}}$. All other chemicals were purchased from Millipore-Sigma (St. Louis, MO, USA) unless otherwise stated.

Cells, transfection and reagents. iSLK.219 cells were maintained in DMEM supplemented with $10 \%$ FBS, $1 \%$ penicillin-streptomycin-L-glutamine solution, $10 \mu \mathrm{g} / \mathrm{ml}$ puromycin, $400 \mu \mathrm{g} / \mathrm{ml}$ hygromycin B, and $250 \mu \mathrm{g} / \mathrm{ml} \mathrm{G} 418$. iSLK cells were obtained from Dr. Don Ganem (Novartis Institute for Biomedical Research) and were maintained in DMEM supplemented with 10\% FBS, $1 \%$ penicillin-streptomycin-L-glutamine solution and $10 \mu \mathrm{g} / \mathrm{ml}$ puromycin. BC-1 and BC-3 cell lines were obtained from ATCC (Manassas, VA, USA), expanded to obtain early passage stocks, and stored. The BCBL-1 cell line was also obtained from Dr. Ganem (University of California San Francisco). BC-1, BC-3, and BCBL-1 cell lines were cultured in RPMI 1640 medium supplemented with $15 \%$ FBS and $1 \%$ penicillin-streptomycin-L-glutamine solution. TREx BCBL1 cells, which can induce Flagx3-HAx3-K-Rta in tetracycline inducible manner, was prepared before (28) were maintained in RPMI 1640 medium supplemented with 10\% FBS, 1\% penicillinstreptomycin-L-glutamine, $250 \mu \mathrm{g} / \mathrm{ml}$ hygromycin $\mathrm{B}$, and $100 \mu \mathrm{g} / \mathrm{ml}$ blasticidin. Plasmids or siRNAs were transfected with Lipofectamine reagent (Thermo Fisher) or Lipofectamine RNAiMax reagent (Thermo Fisher) respectively according to the manufacturer's protocol.

Capture Hi-C. KSHV Capture $\mathrm{Hi}-\mathrm{C}(\mathrm{CHi}-\mathrm{C})$ was performed using a robust in situ $\mathrm{CHi}-\mathrm{C}$ protocol with kitted reagents from Arima Genomics (San Diego, CA, USA) based on methods described for in situ $\mathrm{Hi}-\mathrm{C}(89,90)$, CHi-C (91-93), and as described previously (28). Briefly, cells were 
crosslinked with $2 \%$ formaldehyde, lysed, and the genomic DNA digested with a cocktail of 4cutter restriction endonucleases by incubation for 30 minutes at $37^{\circ} \mathrm{C}$. The $5^{\prime}$-overhangs were then filled in and labeled with biotinylated dATP (biotin-14-dATP) by incorporation with Klenow fragment of DNA polymerase I (incubation for 45 minutes at $25^{\circ} \mathrm{C}$ ). Ligation of the spatially proximal blunt-ended fragments was then performed with T4 DNA ligase (incubation for 15 minutes at $25^{\circ} \mathrm{C}$ ). The formaldehyde crosslinks were reversed and the proximally-ligated, chimeric DNA products were purified with Agencourt AMPure XP paramagnetic beads (Beckman Coulter, Brea, CA, USA). The DNA was then fragmented to an average size of $400 \mathrm{bp}$ with a Covaris E220 Focused-ultrasonicator (Covaris, Inc., Woburn, MA, USA) and size-selected to have a fragment size distribution of $200-600$ bp with AMPure XP beads. The biotin-labeled ligation products were then selectively enriched by affinity capture with streptavidin magnetic beads (Arima Enrichment Beads). Subsequently, sequencing libraries were prepared with the Kapa HyperPrep Kit with Library Amplification Module (Roche, Basel, Switzerland) using a modified protocol for on-bead end repair, dA-tailing, and ligation of Illumina TruSeq sequencing adaptors.

The KSHV CHi-C library was then prepared from the Hi-C libraries as previously described (28). Briefly, target enrichment for KSHV genomic content was performed by solution hybridization with a custom-designed KSHV genomic capture probe library (xGen Lockdown Probes; Integrated DNA Technologies, Inc., Coralville, IA) (28) and subsequent capture of the hybridized targets with streptavidin beads (DynaBeads MyOne Streptavidin C1; Thermo Fisher) according to the manufacturer's standard protocol (Integrated DNA Technologies, Inc., Coralville, IA). The KSHV genome-enriched CHi-C library DNA was eluted and PCR enrichment (12 cycles) performed with high-fidelity KAPA HiFi HotStart DNA Polymerase (Kapa Biosystems, 
512 Inc., Wilmington, MA). Libraries were multiplex sequenced $(2 \times 150 \mathrm{bp}$, paired-end, 50 million 513 mapped reads/mate pairs per sample) on an Illumina Hiseq 4000 sequencing system.

Cleavage Under Targets and Release Using Nuclease (CUT\&RUN). CUT\&RUN (70) was performed essentially by following the online protocol established by Dr. Henikoff's lab with a few modifications. Cells were washed with PBS and wash buffer [(20 mM HEPES-KOH pH 7.5, $150 \mathrm{mM} \mathrm{NaCl}, 0.5 \mathrm{mM}$ Spermidine (Sigma, S2626) and proteinase inhibitor (Roche)]. After removing the wash buffer, cells were captured on magnetic ConA beads (Polysciences, PA, USA), in the presence of $\mathrm{CaCl}_{2}$. Beads/cells complexes were washed with digitonin wash buffer (0.02\% digitonin, $20 \mathrm{mM}$ HEPES-KOH pH 7.5, $150 \mathrm{mM} \mathrm{NaCl,} 0.5 \mathrm{mM}$ Spermidine and 1x proteinase inhibitor) 3 times, aliquoted, and incubated with specific antibodies in $250 \mu \mathrm{L}$ volume. The antibodies used in this study were: rabbit monoclonal anti-CHD4 (Cell Signaling, D4B7; 1:50); rat monoclonal anti-LANA (Millipore-Sigma, clone LN53; 1:50); rabbit anti-ADNP polyclonal (Invitrogen, PA5-52286; 1:100); rabbit monoclonal anti-Acetylated Histone H3 lysine 27 (Cell Signaling, D5E4; 1:100), rabbit monoclonal anti-H3 monomethyl lysine 4 (Cell Signaling,

D1A9; 1:100), and rabbit monoclonal anti-H3 tri-methyl lysine 27 (Cell Signaling, C36B11; 1:100). After incubation, unbound antibody was removed by washing with digitonin wash buffer 3 times. Beads were then incubated with recombinant pAG-MNase, which was purified from

E.coli (Supplementary Fig. 9), in $250 \mu$ l digitonin wash buffer at $1.0 \mu \mathrm{g} / \mathrm{mL}$ final concentration for one hour at $4^{\circ} \mathrm{C}$ with rotation. Unbound pAG-MNase was removed by washing with digitonin wash buffer 3 times. Pre-chilled $2 \mathrm{mM} \mathrm{CaCl}_{2}$ containing digitonin wash buffer $(200 \mu \mathrm{L})$ was added 533 to beads and incubated on ice for $30 \mathrm{~min}$. The pAG-MNase digestion was halted by the addition 534 of $200 \mu \mathrm{l} 2 \times$ STOP solution (340 mM NaCl, 20 mM EDTA, 4 mM EGTA, $50 \mu g / m l$ RNase A, 50 
$535 \mu \mathrm{g} / \mathrm{ml}$ glycogen). The beads were incubated with shaking at $37^{\circ} \mathrm{C}$ for $10 \mathrm{~min}$ in a tube shaker at $536500 \mathrm{rpm}$ to release digested DNA fragments from the insoluble nuclear chromatin. The 537 supernatant was collected after centrifugation $\left(16,000 \times \mathrm{g}\right.$ for 5 min at $\left.4^{\circ} \mathrm{C}\right)$ and placed on a 538 magnetic stand. DNA was extracted using the NucleoSpin kit (Takara Bio, Kusatsu, Shiga, 539 Japan). Sequencing libraries were then prepared from $3 \mathrm{ng}$ of CUT\&RUN DNA with the Kapa 540 HyperPrep Kit (Roche) according to the manufacturer's standard protocol. Libraries were 541 multiplex sequenced ( $2 \times 150 \mathrm{bp}$, paired-end) on an Illumina HiSeq 4000 sequencing system to 542 yield $\sim 15$ million mapped reads per sample. When necessary, E. coli genomic DNA read from a 543 pAG-MNase incubation were used to normalize data as described previously (70).

545 Genomic data analysis.

546 The HiC-pro 2.11.1 pipeline (94) was used to align sequences from the Hi-C experiments against 547 a combined assembly of reference genomes; the human hg19 (GRCh37) and KSHV 548 (NC_009333.1). The reads were filtered for only uniquely mapped reads pairs by identifying 549 intersection of each read-end, and the valid reads were provided by removing reads with self550 circle, dangling-end, error, extra dangling-end, too short, too large, duplicated, and random

551 breaks. The valid reads were stored as matrices and binned with resolution of $10000 \mathrm{bp}$. Iterative 552 Correction and Eigenvector decomposition (ICE) normalization was used to treat the data with 553 default parameters. The normalized counts were filtered to keep only the counts that mapped to 554 both the human and KSHV genome by using Python with Pandas library. The results were 555 visualized as dot plots using Matplotlib library. 
556 The normalized counts were also used as input in subsequent analyses. In the analysis of KSHV 557 episome localization near centromeres, centromeres of human chromosome (hg19) were 558 downloaded from the UCSC Table Browser with a filter of 'centromere' as gap type. For each 559 chromosome, the sum of all counts was compared to both the sums of the counts in the regions 560 near the centromere at the 5' and 3' sides, both of which are $1 \%$ the size of the chromosome 561 (the values were first normalized as per 100,000 bp before the comparisons). The similarity of $562 \mathrm{CHi}-\mathrm{C}$ chimeric sequence reads among $\mathrm{BC}-1, \mathrm{BC}-3$, and $\mathrm{BCBL}-1$ were performed as follows. 563 Genomic regions with normalized counts $>0$ were extracted and then the intersect and union 564 regions were determined using Intervene v0.6.4 (95). The similarity percentage was calculated 565 based on the Jaccard similarity index.

566 FASTQ files for the capture Hi-C experiments were processed through the HiCUP (v0.7.4) 567 pipeline (96) using a combined human hg19 (GRCh37) and KSHV (NC_009333.1) genome. 568 Valid interaction products called by HiCUP were converted into Juicebox (97) input format (.hic 569 file), which stores the normalized and un-normalized contact matrices as a highly compressed 570 binary file, by using a series a scripts provided by HiCUP (hicup2homer) and HOMER 571 (makeTagDirectory and tagDir2hicFile) (98). Juicebox was utilized to facilitate adjustments of 572 resolution and normalization, intensity scaling, zooming, and addition of annotation tracks. CUT\&RUN sequence reads were aligned to the human hg38 reference genome and 574 reference KSHV genome sequence (Human herpesvirus 8 strain: GQ994935.1) with Bowtie2 575 v2.3.5.1 (99) and/or HISAT2 v2.1.0 (100). MACS2 (Model-based Analysis of ChIP-Seq) 576 v2.1.1.1.20160309 was used for detecting peaks (101) following the developer's manual. Peaks 577 and read alignments were visualized using the Integrated Genome Browser (IGB) (102). 578 Heatmaps and average profile plots were drawn from bed files created by MACS and bam files 
579 using R package, ngsplot v2.63 (103). After subtracting the minimum value of average profile 580 as background signal, relative average profile plot was drawn. Accumulation index was 581 calculated as proportion of area under the curve.

583 Single cell RNA sequencing data analysis. Single cell data was analyzed with the Cell Ranger 584 v2.1 pipeline (10x Genomics). The pipeline included alignment to the hg38 human reference 585 genome and human herpesvirus 8 strain (GQ994935.1) reference genome, t-distributed 586 stochastic neighbor embedding (tSNE), and K-means clustering. Read count matrices obtained 587 from the pipeline were normalized using log normalization method with "Seurat" R package 588 (104). To perform correlation analysis, the expression of the KSHV genes was summarized, log 589 transformed and resulting values divided by 14 equal intervals to have more than 50 cells in 590 each interval. The median value of gene expression was calculated for the cells in each interval. 591 The median values were used to analyze and visualize the correlation of host cellular and viral 592 gene expression.

594 Immunofluorescence staining analyses. iSLK cells latently infected with BAC16-Wt were 595 seeded onto glass coverslips and transfected with pLenti-Flag-mCHD4-myc WT or pLenti-Flag596 mCHD4-myc mutant using Lipofectamine 2000 reagent according to the manufacturer's 597 protocol. pLenti-Flag-mCHD4-myc was obtained from a commercial source (Origene, MD, USA). 598 Point mutations were inserted by synthesizing DNA fragments that contain intended mutations 599 and replaced at SexAl-Agel restriction sites with In-FusionHD (Takara Bio, Japan). At 24 hrs 600 after transfection, $1 \mu \mathrm{g} / \mathrm{ml}$ of doxycycline and $20 \mathrm{ng} / \mathrm{ml}$ of $12-O-T e t r a d e c a n o y \mid p h o r b o l-13-a c e t a t e$ 
601 (TPA) were added, and cells were cultured for a further 26 hrs in the presence of doxycycline 602 and TPA. The cells were then fixed with $4 \%$ paraformaldehyde, permeabilized with $0.2 \%$ Triton $603 \mathrm{X}-100$, and labeled with anti-RNAPII mouse monoclonal antibody (Millipore-Sigma, clone 604 CTD4H8, 1:100) and anti-FLAG rabbit polyclonal antibody (Sigma, F7425, 1:100), followed by 605 Alexa 555-anti mouse IgG and Alexa 647-anti rabbit IgG (Thermo Fisher). Nuclei were 606 counterstained with $1 \mu \mathrm{g} / \mathrm{ml}$ of Hoechst 33342 (Thermo Fisher). The labeled cells were observed 607 with a Keyence BZ-X710 fluorescence microscope (Keyence, Osaka, Japan) with standard 608 DAPI, GFP, TRITC and Cy5 filter sets (Chroma Technologies, Bellows Falls, VT, USA).

609 DNA-FISH combining with LANA immunostaining. BCBL-1 cells were washed with PBS 610 twice and spotted on coverslips and fixed with $4 \%$ formaldehyde-PBS for 10 min at RT. Cells 611 were subsequently incubated with PBS with $100 \mathrm{mM}$ glycine to quench residual formaldehyde. 612 Cells were treated with $0.1 \%$ Triton-X and $0.05 \%$ SDS with RNaseA for 15 min at $37^{\circ} \mathrm{C}$. 613 Coverslips were washed with PBS twice, $70 \%$ ethanol once, $85 \%$ ethanol once, and $100 \%$ 614 ethanol once and dried completely. Cy5-conjugated CENPB-Cy5 PNA probe was obtained from 615 a commercial source (PNA Bio, Thousand Oaks, CA, USA) and coverslips were incubated with 616 hybridization buffer (200 nM PNA probe, 2xSSC, 20\% Dextran, $1 \mathrm{mg} / \mathrm{mL}$ yeast tRNA, $60 \%$ 617 formamide) on a glass slide on a heating plate at $57^{\circ} \mathrm{C}$ for $10 \mathrm{~min}$ and then $37^{\circ} \mathrm{C}$ for another 30 618 min. Utilization of PNA probes avoids harsh conditions often used for a larger DNA hybridization, 619 which we found to produce unacceptable noise and not readily compatible with subsequent 620 protein staining. The coverslip was washed with warmed $0.1 \%$ Tween 20 in PBS $\left(57^{\circ} \mathrm{C}\right)$ in 6621 well plates for 3 times and PBS once. Primary antibody was incubated for LANA staining in PBS 622 with $1 \mathrm{mg} / \mathrm{mL}$ yeast tRNA. After washing with PBS 3 times, secondary antibody (Alexa 488-anti 623 Rat lgG) was incubated in PBS with yeast tRNA for $1 \mathrm{~h}$ at $37^{\circ} \mathrm{C}$. After washing three times with 
624 PBS, the coverslips were mounted with antifade reagent (Thermo Fisher), and images were 625 captured with a Keyence microscope.

3D Fluorescence microscopy and quantitative image analysis. Widefield 3D fluorescence

627 imaging was performed on a Keyence BZ-X710 fluorescence microscope (Keyence, Osaka, 628 Japan) equipped with live-cell environmental chamber. Cells fluorescently labeled as previously 629 described were observed with standard DAPI, GFP, TRITC and Cy5 filter sets (Chroma 630 Technologies, Bellows Falls, VT, USA). High resolution image stacks were acquired in each 631 color channel using Keyence onboard software. Image stacks were converted to greyscale TIF 632 images that were uniformly corrected for haze reduction, deblurring and contrast enhancement.

Image data were subsequently exported to the Volocity® Multi-Dimensional Imaging 634 Platform (Quorum Technologies Inc., Ontario, Canada) for 3D visualization and quantitative 635 analysis. For image-based cytometry measurements, individual cells within a given field of view 636 were automatically identified and fluorescent objects and/or region of interest in the various color 637 channels were quantified and tabulated. For qualitative assessment of LANA and centromere 638 oligo probes for DNA-FISH, 3D image stacks were similarly compiled and displayed in 3D opacity 639 mode using Volocity Visualization toolset. Reconstructed 3D images were used for distance640 measurement analysis: briefly, fluorescent LANA dots (green) and DNA centromeres (purple) in 641 individual cell nuclei were automatically identified in Volocity Quantitation. The minimum 642 distance from each centromere to the nearest LANA dot could then be extracted from the 643 statistical measurements and displayed as histograms for subsequent analysis. 
Lentivirus production and transduction. For CHD4 knock-down experiments, the following shRNA sequences from Sigma pLKO.1 shRNA libraries were used: shRNA\#1: CCTTACTAGAATTGGTGTTAT; shRNA\#2: GCTGACACAGTTATTATCTAT. Lentiviruses generated from the CHD4-expressing lentivector, CHD4-targeting shRNAs, and non-targeting scramble shRNA (Addgene, \#1864) were produced in 293T cells. The vectors were cotransfected with psPAX2 (Addgene, \#12260) and pMD2.G (Addgene, \#12259) into 293T cells using polyethylenimine (PEI). Supernatants were collected 48 and 72 hrs post-transfection. Cells were infected with lentiviruses in the presence of $8 \mu \mathrm{g} / \mathrm{ml}$ polybrene and subsequently incubated for 72 hrs to allow for protein expression or knock-down. To generate CHD4 knock-down cell lines, 293T cells were infected with lentivirus for $24 \mathrm{hrs}$. Cells were then cultured in selection medium containing $1 \mu \mathrm{g} / \mathrm{ml}$ puromycin for two weeks to obtain stable CHD4 knock-down cell lines.

\section{Preparation of purified KSHV and de novo infection. iSLK.219 cells latently infected with} recombinant $\mathrm{KSHV}$ were cultured in eight to ten $150 \mathrm{~mm}$ culture dishes until $80 \%$ confluent. For reactivation, cultures were re-fed with complete DMEM (without selection drugs) containing $0.3 \mathrm{mM}$ sodium butyrate and $1 \mu \mathrm{g} / \mathrm{ml}$ doxycycline. The cells were further cultured for 5 days in the presence of sodium butyrate and doxycycline. The culture supernatant was centrifuged at $300 \times \mathrm{g}$ for $10 \mathrm{~min}$, and then passed through a $0.8-\mu \mathrm{m}$ filter to remove cellular debris, and then viral particles were concentrated by ultracentrifugation at $25,000 \mathrm{rpm}$ for $2 \mathrm{hrs}$ at $4{ }^{\circ} \mathrm{C}$ with a Beckman SW28 rotor. The viral precipitates were resuspended in $500 \mu \mathrm{l}$ of DMEM along with the residual $\sim 500 \mu \mathrm{l}$ media in the centrifuge tube and stored at $-80^{\circ} \mathrm{C}$ until use. For de novo infection, CHD4-KD 293T cells were seeded at $4 \times 10^{5}$ cells/well in 6-well plates. After overnight 
668 culture, viral stocks were added to the cultures and the infection was allowed to proceed for $66924 \mathrm{hrs}$. Cells were monitored for GFP expression and RNA was purified using the RNeasy Mini 670 Kit (Qiagen, Venlo, Netherlands).

Flow Cytometry. 293T cells were transfected with 10 pmol CHD4 siRNA using Lipofectamine RNAiMax reagent (Thermo Fisher) for 48 hrs. Cells were infected with r.219 virus for 96 hrs in DMEM media containing 5\% FBS. After that, cells were trypsinized and washed with PBS two

675

676

677

678

679

680

681

682 683

684

685

686

687

688

689

times followed by fixation with $2 \%$ PFA containing $1 \mathrm{mM}$ EDTA for 10 minutes at $37^{\circ} \mathrm{C}$. Cells were then washed with PBS twice and resuspended in PBS containing $1 \mathrm{mM}$ EDTA. Cells were passed through a $0.45-\mu \mathrm{m}$ strainer to obtain single cells. A BD "Fortessa" cytometer was used for FACS analysis, and FlowJo software (Tree Star) was used for data analysis.

Purification of recombinant protein. Spodoptera frugiperda Sf9 cells (Millipore) were maintained in Ex-Cell 420 medium (Sigma), and recombinant baculoviruses were generated with the Bac to bac baculovirus expression system as previously described $(20,105)$. Transfer plasmid, pFAST-BAC1 vector was modified by inserting a Flag tag at the $\mathrm{N}$-terminus, and CHD4, ORF57, p65, and Luciferase cDNAs were cloned into the Cpol (Rsrll) site. The cDNA of ADNP, which also include $\mathrm{C}$-terminal His tag was synthesized and cloned into BamHI and Pstl restriction enzyme sites by Gene assembly (NEB). Recombinant baculovirus bacmid DNA was transfected into Sf9 cells by using polyethylenimine (Sigma), and recombinant viruses were subsequently amplified twice. Expression of recombinant proteins was confirmed by immunoblotting with antiFlag monoclonal antibody (Sigma). Large-scale cultures of Sf9 cells $(100 \mathrm{ml})$ were infected with 
690

691

692

693

694

695

696

697

698

699

700

701

702

703

704

705

706

707

708

709

710

711

recombinant baculovirus at a multiplicity of infection (MOI) of 0.1 to 1.0 , and cells were harvested $48 \mathrm{hrs}$ after infection. Recombinant proteins were purified as described previously (106). The purity and amount of protein were measured by SDS-PAGE and Coomassie blue staining, using bovine serum albumin (BSA) as a standard.

In vitro interaction assays. Baculoviruses expressing Flag-CHD4, His-ADNP, or Flag-LANA were co-infected in SF9 cells and purified by Flag tag capture in the presence of $500 \mathrm{mM} \mathrm{NaCl}$ and $10 \%$ glycerol. Purified complex was re-suspended in the binding buffer $(20 \mathrm{mM} \mathrm{HEPES} \mathrm{[pH}$ 7.9], $150 \mathrm{mM} \mathrm{NaCl}, 1 \mathrm{mM}$ EDTA, 4 mM MgCl $2,1 \mathrm{mM}$ dithiothreitol, $0.02 \%$ NP-40, $10 \%$ glycerol supplemented with $1 \mathrm{mg} / \mathrm{ml} \mathrm{BSA}$, and $1 \times$ protease inhibitor cocktail) and the antibody was incubated at $1: 100$ dilution for $1 \mathrm{hrs}$ at $4^{\circ} \mathrm{C}$ to form an immunocomplex. The immunocomplex was captured with $10 \mu \mathrm{L}$ of protein $\mathrm{A} / \mathrm{G}$ magnetic beads mixture. Beads were washed three times with binding buffer and subjected to SDS-PAGE after eluting proteins in sample buffer. The interaction was probed by immunoblotting with anti-CHD4, anti-ADNP, or anti-LANA antibody.

For RNA or DNA pull-down, a final concentration of $100 \mathrm{nM}$ of each biotinylated RNA fragment (e.g. $1.3 \mu \mathrm{g}$ for full length PAN RNA transcribed in vitro or $2.6 \mu \mathrm{g}$ of dsDNA generated by PCR with 5'-biotinylated primer) and purified proteins (100 nM in final) was incubated in RNAbinding buffer (50 mM HEPES [pH 7.9], $150 \mathrm{mM} \mathrm{KCl,} \mathrm{5 \%} \mathrm{glycerol,} \mathrm{0.02 \%} \mathrm{NP-40,} 100 \mu \mathrm{g} / \mathrm{mL}$ yeast tRNA, $2 \mathrm{mM} \mathrm{MgCl}$ ) supplemented with RNAse inhibitor in $40 \mu \mathrm{L}$ reaction for $1 \mathrm{hrs}$ at $4^{\circ} \mathrm{C}$. Biotinylated RNA or dsDNA was captured with streptavidin conjugated magnetic beads, washed with binding buffer 3 times, and proteins were eluted in sample buffer. Precipitated proteins were 
712 visualized by immunoblotting with either anti-Flag antibody (Sigma) or specific antibody as 713 indicated in figure legends.

Construction of mini-Turbo KSHV BAC16. Recombinant KSHV was prepared by following a protocol for En passant mutagenesis with a two-step markerless red recombination technique (107). Briefly, the codon optimized mini-TurbolD coding sequence (65), which also encodes the 3x Flag tag was first cloned into a pBS SK vector (Thermo Fisher, Waltham, MA USA). The pEPkan-S plasmid was used as a source of the kanamycin cassette, which includes the I-Scel restriction enzyme site at the 5'-end of the kanamycin coding region (107). The kanamycin cassette was amplified with primer pairs with homology arms and cloned into the mini-TurbolD coding region at a unique restriction enzyme site. The resulting plasmid was used as a template for another round of PCR to prepare a transfer DNA fragment for markerless recombination with BAC16 (108). Recombinant BAC16 clones with insertion and also a deletion of the kanamycin cassette in the BAC16 genome were confirmed by colony PCR with appropriate primer pairs. Recombination junctions and adjacent genomic regions were amplified by PCR and the resulting PCR products were directly sequenced with the same primers to confirm in-frame insertion of the mini-TurboID cassette into the BAC16 DNA for mini-TurbolD-ORF57 and -LANA. Insertion of PAN RNA MRE mutations was performed by using primer pairs that encode the intended mutations. Primer pairs were used to amplify the kanamycin cassette and recombination, 731 deletion of kanamycin cassette, confirmation of mutations was performed as described above. 732 The resulting recombinant BAC16 was confirmed by restriction enzyme digestions (Hindll and $733 \mathrm{Bg} / \mathrm{II})$ to determine if there were any large DNA deletions. Two independent BAC16 clones were 734 generated for each mini-TurbolD tagged recombinant KSHV virus as biological replicates and 
735 one of the clones was used for protein ID. Entire BAC16 DNAs were subsequently sequenced 736 by NGS on an Illumina MiSeq System.

737 Quantification of viral copy number. Two hundred microliters of cell culture supernatant were 738 treated with DNase I $(12 \mu \mathrm{g} / \mathrm{ml})$ for $15 \mathrm{~min}$ at room temperature to degrade unencapsidated 739 DNA. This reaction was stopped by the addition of EDTA to $5 \mathrm{mM}$ followed by heating at $70^{\circ} \mathrm{C}$ 740 for 15 min. Viral genomic DNA was purified using the QIAamp DNA Mini Kit according to the 741 manufacturer's protocol and eluted in $100 \mu \mathrm{l}$ of buffer AE. Four microliters of the eluate were 742 used for real-time qPCR to determine viral copy number, as described previously (105).

Real-time RT-PCR. Total RNA was isolated using the Quick-RNA miniprep kit (Zymo Research, 745 Irvine, CA, USA). First-strand cDNA was synthesized using the High Capacity cDNA Reverse 746 Transcription Kit (Thermo Fisher, Waltham, MA USA). Gene expression was analyzed by 747 realtime qPCR using specific primers for KSHV ORFs designed by Fakhari and Dittmer (109). 748 We used $18 \mathrm{~S}$ ribosomal RNA or GAPDH as an internal standard to normalize viral gene 749 expression.

751 Western blotting. Cells were lysed in IP lysis buffer (25 mM Tris-HCl pH 7.4, $150 \mathrm{mM} \mathrm{NaCl}, 1 \%$ 752 NP-40, 1 mM EDTA, 5\% glycerol) containing protease inhibitors (Roche, Basel, Switzerland). 753 Total cell lysates $(25 \mu \mathrm{g})$ were boiled in SDS-PAGE loading buffer and subjected to SDS-PAGE 754 and subsequently transferred to a polyvinylidene fluoride membrane (Millipore-Sigma, St. Louis, 755 MO, USA) using a semidry transfer apparatus (Bio-Rad, Hercules, CA, USA). The streptavidin756 HRP conjugate was used at 1:3000 dilution. Final dilution of the primary antibody was 1:3,000 
757 for anti-Flag mouse antibody, 1:1,000 for anti-LANA rat antibody, anti-CHD4 rabbit, and anti758 ADNP rabbit antibodies. Washing membranes and secondary antibody incubations were 759 performed as described previously (105).

Affinity purification of biotinylated proteins. KSHV LANA-mTID cells were incubated with

$762100 \mu \mathrm{M}$ D-biotin for 60 mins at $37^{\circ} \mathrm{C}$. To stop the reaction, cells were incubated at $4^{\circ} \mathrm{C}$ for 60 763 mins. Cells were washed with cold PBS three times followed by lysis with SUMO lysis buffer 764 (Tris-CI PH 6.8, 1\% SDS and 10\% glycerol). Lysates were immediately boiled for 10 min, and 765 protein was quantified by Bradford assay (Bio-Rad). The biotinylation signal was confirmed by 766 western blotting using Strep-HRP.

767 Affinity purification was performed with streptavidin-coated magnetic beads (Thermo-Fisher). 768 Briefly, $150 \mu$ magnetic beads/sample were pre-washed with RIPA lysis buffer (150 mM NaCl, 7695 mM EDTA (pH 8), 50 mM Tris ( $\mathrm{pH} 8$ ), 1\% NP-40, 0.5\% sodium deoxycholate, $0.1 \%$ SDS) 3 770 times. A total of $3 \mathrm{mg}$ of whole cell lysate was incubated with pre-washed streptavidin beads 771 after diluting with RIPA buffer for 10 times, at room temperature for $1 \mathrm{~h}$ with rotation. The beads 772 were collected using a magnetic stand and washed three times with wash buffer according to 773 the manufacturer's protocol. Finally, beads were resuspended in $200 \mu$ l of wash buffer and sent 774 to UC Davis Proteomics core for on bead digestion and LC-MS/MS analysis.

MS sample preparation. Protein samples on magnetic beads were washed four times with 200 $777 \mu \mathrm{l}$ of $50 \mathrm{mM}$ ammonium bicarbonate $(\mathrm{AMBIC})$ with a twenty-minute shake time at $4^{\circ} \mathrm{C}$ in between 778 each wash. Roughly $2.5 \mu \mathrm{g}$ of trypsin was added to the beads and AMBIC and the samples were 
779 digested overnight at $800 \mathrm{rpm}$ shake speed. After overnight digestion, the supernatant was 780 removed, and the beads were washed once with enough $50 \mathrm{mM} \mathrm{AMBIC}$ to cover them. After 20 781 minutes at a gentle shake the wash was removed and combined with the initial supernatant. The 782 peptide extracts were reduced in volume by vacuum centrifugation and a small portion of the 783 extract was used for fluorometric peptide quantification (Thermo Scientific Pierce). One 784 microgram of sample based on the fluorometric peptide assay was loaded for each LC-MS 785 analysis.

Digested peptides were analyzed by LC-MS/MS on a Thermo Scientific Q Exactive 787 Orbitrap Mass spectrometer in conjunction with Proxeon Easy-nLC II HPLC (Thermo Scientific) 788 and Proxeon nanospray source. The digested peptides were loaded on a 100 micron $\times 25 \mathrm{~mm}$ 789 Magic C18 100Å $5 U$ reverse phase trap where they were desalted online before being separated 790 using a 75-micron x 150 mm Magic C18 200Å 3U reverse-phase column. Peptides were eluted using a 60-minute gradient with a flow rate of $300 \mu \mathrm{l} / \mathrm{min}$. An MS survey scan was obtained for 792 the $\mathrm{m} / \mathrm{z}$ range $300-1600, \mathrm{MS} / \mathrm{MS}$ spectra were acquired using a top 15 method, where the top 79315 ions in the MS spectra were subjected to HCD (High Energy Collisional Dissociation). An 794 isolation mass window of $2.0 \mathrm{~m} / \mathrm{z}$ was used for the precursor ion selection, and normalized 795 collision energy of $27 \%$ was used for fragmentation. A fifteen second duration was used for the 796 dynamic exclusion.

MS/MS analysis. Tandem mass spectra were extracted and charge state deconvoluted by 799 Proteome Discoverer (Thermo Scientific). All MS/MS samples were analyzed using X! Tandem 800 (The GPM, thegpm.org; version X! Tandem Alanine (2017.2.1.4)). X! Tandem was set up to 801 search the Human and Kaposi's Sarcoma Herpes virus database (149182 entries) assuming the 
802 digestion enzyme trypsin. $X$ ! Tandem was searched with a fragment ion mass tolerance of 20 803 PPM and a parent ion tolerance of 20 PPM. Carbamidomethyl of cysteine and selenocysteine 804 was specified in X! Tandem as a fixed modification. Glu->pyro-Glu of the N-terminus, ammonia805 loss of the $\mathrm{N}$-terminus, gln->pyro-Glu of the $\mathrm{N}$-terminus, deamination of asparagine and 806 glutamine, oxidation of methionine and tryptophan and dioxidation of methionine and tryptophan 807 were specified in X! Tandem as variable modifications.

Scaffold (version Scaffold_4.8.4, Proteome Software Inc., Portland, OR) was used to validate MS/MS-based peptide and protein identifications. Peptide identifications were accepted if they could be established at greater than $98.0 \%$ probability by the Scaffold Local FDR 811 algorithm. Peptide identifications were also required to exceed specific database search engine 812 thresholds. Protein identifications were accepted if they could be established at greater than $8135.0 \%$ probability to achieve an FDR less than $5.0 \%$ and contained at least 2 identified peptides. 814 Protein probabilities were assigned by the Protein Prophet algorithm (110). Proteins that 815 contained similar peptides and could not be differentiated based on MS/MS analysis alone were 816 grouped to satisfy the principles of parsimony. Proteins sharing significant peptide evidence 817 were grouped into clusters.

Pathway analysis. The proteins identified to be interacting with ORF57 were used for gene ontology analysis. The top gene ontology processes were enriched by the Metascape web821 based platform, and the Metascape software was used for gene ontology analysis (111). 
823 Statistical analysis. Results are shown as mean \pm SD from at least three independent 824 experiments. Data were analyzed using unpaired Student's $t$ test, or ANOVA followed by Tukey's 825 HSD test. A value of $p<0.05$ was considered statistically significant.

Code availability. All custom codes used to analyze data and generate figures are available 828 upon request.

Data availability. Data can be accessed via the Source Data files and Supplementary tables. 831 The genomic data discussed in this publication have been deposited in NCBI's GEO Database 832 under unified Super Series GSE163695 with subseries GSE163098 for CUT\&RUN and 833 GSE163694 for CHi-C.

Acknowledgement. This research was supported by public health grants from the National 836 Cancer Institute (CA225266, CA232845), the National Institute of Dental and Craniofacial 837 (DE025985), and the National Institute of Allergy and Infectious Disease (Al147207) to Y.I. The 838 Genomics Shared Resource is supported by the UC Davis Comprehensive Cancer Center 839 Support Grant (CCSG) awarded by the National Cancer Institute (NCI P30CA093373).

\section{Author Contributions.}

842 A.K., Y.L., M.C., and Y.I. designed and performed experiments, analyzed data and prepared 843 figures. Y.Y., C.C., R.R.D., K.N., and A.M. ran genomic pipelines, performed statistical analyses, 
844 and visualized genomic data sets. M.S. prepared enzyme digested protein samples and performed 845 proteomics data analyses. R.R.D. and C.G.T. prepared sequencing libraries and performed initial 846 bioinformatics analyses. K.I.N and R.R.D. prepared single cell transcriptomics libraries, and A.M. 847 wrote custom codes and analyzed single-cell sequence data sets. A.K., Y.L., K.W., C.I., and Y.I. 848 prepared BAC stable cell lines, and performed molecular and biochemical studies. YI generated 849 recombinant BACs. F.C. and K.I.N. prepared slides and performed imaging analyses. V.M., M.S., 850 and Z.M.Z. contributed key reagents. A.K, Y.L., Y.Y., C.C., M.S., F.C., R.R.D., C.G.T., K.N., K.I.N., 851 V.M., Z.M.Z. and Y.I. analyzed corresponding data sets. Y.I. wrote the manuscript and all authors 852 edited the manuscript.

853

854 Competing financial interests.

855 The authors have declared that no conflicts of interest exist.

856

857 Materials \& Correspondence.

858 Correspondence and request for materials should be addressed to M.C. (e-mail: 859 mcampbell@ucdavis.edu) or Y.I. (email: yizumiya@ucdavis.edu). 
861 Figure legends

862 Fig. 1 KSHV episome-tethering sites in KSHV positive cell lines. (a) Schematic workflow 863 for Capture-HiC (CHi-C). (b) KSHV episome-docking sites on host cell chromosomes. 864 CHi-C chimeric DNA ligation products composed of sequences derived from the KSHV and 865 human genomes were mapped in three naturally-infected PEL cells. For BCBL-1 cells, three 866 biological replicates were performed with nearly identical results (similarity 0.95). M, 867 mitochondrial chromosome. ICE-corrected profiles depicting sums of filtered read counts binned 868 at $10 \mathrm{~kb}$ resolution are shown. (c) Selected chromosome 1 in zoomed view. Chromosome 1 869 dot plots were depicted, which displays enrichment of sequence reads near the centromere. Red 870 arrows indicate the position of the centromere. Extended panels for all other individual 871 chromosome for three cell lines are presented in Supplementary Fig. 1a-c. (d) KSHV episomes 872 preferentially localize near the centromere. KSHV episome contacts were calculated in 873 BCBL-1 cells for sequence reads in 1\% (length of the respective chromosome) of the 5'-and 3'874 centromere and compared with the average of the total chromosome read count. (e) LANA 875 protein locates near the centromere. DNA-FISH was used to visualize the location of the 876 centromere (red) and KSHV episomes were indirectly visualized by staining LANA dots (Green) 877 in BCBL-1 cells. The centromere and LANA were probed using a Cenp probe and anti-LANA 878 antibody respectively with IFA. The two signals were frequently co-localized, and 3D view and 879 their image analyses are presented in Supplementary Fig. 2. (f) Jaccard statistics and Venn 880 diagram. Venn diagram shows percent similarity of KSHV episome-tethering positions among 881 BC-1, BC-3 and BCBL-1 cells. 
Fig. 2 LANA interacts with the ChAHP complex. (a) A schematic diagram of preparation of recombinant KSHV infected iSLK cells. A mini-TurbolD (mTID) was fused to the N-terminus of the LANA open reading frame to generate KSHV LANA-mTID. KSHV LANA-mTID was transfected into iSLK cells followed by production of viral particles by stimulating cells with doxycycline $(1 \mu \mathrm{g} / \mathrm{ml})$ and sodium butyrate $(3 \mathrm{mM})$ for 5 days. Virus was used to infect iSLK cells and cells were selected with hygromycin $(1 \mathrm{mg} / \mathrm{ml})$ to obtain an iSLK-LANA mTID stable cell line. (b) Experimental design for preparing samples for protein ID. ISLK-LANA mTID cells were left un-incubated (-) or incubated $(+)$ with D-Biotin $(500 \mu \mathrm{M})$ for $1 \mathrm{hr}$. Unincubated (-) cells were used as control samples. Each protein ID was performed in three biological replicates. (c) Volcano plot depicting proteins in close proximity to LANA. The volcano plot represents proteins identified in close proximity to LANA. Proteins with an abundance Log2 FC of greater than or equal to 1 and p-value less than 0.05 were selected and are shown by blue box. T-test was used for calculating the p-value. Purple color; ChAHP components. Red color; previously identified proteins as LANA interacting proteins. (d) LANA interacts with CHD4 and ADNP in vitro. LANA, CHD4 and ADNP complex, which consists of Flag-LANA (gold arrow), Flag-CHD4 (blue arrow), and His-ADNP (green arrow) was prepared by co-infected three recombinant baculoviruses, and the protein complex was isolated in the presence of $500 \mathrm{mM} \mathrm{NaCl}$ and $10 \%$ glycerol with affinity purification. The authenticity of respective protein band was confirmed by immunoblotting with specific antibodies. Coomassie staining is shown. An equimolar amount $(100 \mathrm{nM})$ of the three proteins were incubated in binding buffer and immunoprecipitated with the specific antibodies of one another. Ten percent of the reaction before immunoprecipitation was shown as controls. W.B: Western Blotting, Co-IP: Co-immunoprecipitation.

(e) CHD4 interaction site on LANA. Purified GST-tagged LANA deletions, Flag-tagged luciferase and 
906 Flag-tagged CHD4 were prepared from recombinant baculovirus infected Sf9 cells. An equal 907 amount $(1 \mu \mathrm{g})$ of each LANA deletion protein was incubated with full length Flag-tagged 908 luciferase $(1 \mu \mathrm{g})$ or Flag-tagged CHD4 $(1 \mu \mathrm{g})$ in binding buffer and interaction was probed with 909 anti-Flag antibody. LANA deletion proteins used for this GST-pull down assay are presented in 910 Supplementary Fig. 4. (f) LANA co-localizes with CHD4 in BCBL-1. CHD4 and LANA were 911 probed with anti-CHD4 rabbit monoclonal antibody and anti-LANA rat monoclonal antibody, 912 respectively. Images were taken with Keyence fluorescence microscopy. Scale bar $(20 \mu \mathrm{m})$ is 913 shown.

Fig. 3 Association of ChAHP complex binding with KSHV episome-tethering sites. (a) CUT\&RUN analysis for LANA, ADNP, CHD4 and H3K27Ac. The indicated antibodies were 917 used for CUT\&RUN in BCBL-1 cells. CUT\&RUN peaks were visualized with Integrative 918 Genomics Viewer (IGV) and a snapshot of one of the major binding sites (IRF4 super enhancer region) is shown. The number on the left-hand side denotes the height of the peak (e.g., read depth) and the number along the top denotes the positions on chromosome 6. (b) Heatmaps for co-occupancies of LANA with ADNP, CHD4 and H3K27Ac on chromosomes. Heatmap

922 (middle) and average profile (bottom) showing correlation of LANA enrichment (by color intensity and region) with ADNP, CHD4, and H3K27Ac occupancies. Average profile plot summarizing 924 the heatmap (bottom). The lighter green shade represents the standard error (SEM) on the 925 average profile plot. Color keys are shown on the each heatmap (top). (c) Heatmaps for correlation of $\mathrm{CHi}-\mathrm{C}$ with CUT\&RUN signals. The association between $\mathrm{CHi}-\mathrm{C}$ chimeric reads and LANA, CHD4, ADNP and H3K4me1 CUT\&RUN peaks were depicted as heatmap and average profile plot. (d) Accumulation index around CHD4 binding sites. The accumulation 
929 of CHD4 is displayed as a proportion of area under the curve of the relative average profile. 930 Association between distance from CHD4 summit peak and relative chimeric sequence counts 931 is shown as a bar chart.

Fig. 4 LANA, CHD4 and ADNP colocalize on the KSHV genome. (a) CUT\&RUN analysis. The indicated antibodies were used for CUT\&RUN in BCBL-1 cells and sequence reads were mapped to the KSHV genome. An IGV snapshot and KSHV genome map are shown. The number on the left-hand side denotes the height of the peak (e.g., read depth), the number along the top denotes the position on KSHV genome in kilobase $(\mathrm{kb})$ and the number on the right-hand side indicated by arrow denote the height of the peak on terminal repeat region (TR). Selected region is zoomed in to show Ori-RNA and PAN RNA region. Primer used in Fig. 4b is also shown with red arrows and number show the exact location of the amplified region by the primers. (b)

Regulation of CHD4 on the KSHV episome during reactivation. TREx-BCBL-1 cells were reactivated with doxycycline $(1 \mu \mathrm{g} / \mathrm{ml})$ and sodium butyrate $(3 \mathrm{mM})$ for $24 \mathrm{hrs}$. CUT\&RUN was performed on un-reactivated (latency) and reactivated cells for CHD4. CHD4 binding on the PAN RNA promoter region (red arrow in Fig. 4a, right panel) was calculated relative to the input sample ( $n=3$, two biological replicates are shown). (c) Detachment of KSHV episomes by reactivation. BCBL-1 cells were reactivated with doxycycline $(1 \mu \mathrm{g} / \mathrm{ml})$ and TPA $(20 \mathrm{nM})$ for 24 hrs. Chimeric sequence reads for un-reactivated (latency) and reactivated cells were mapped to 948 the host chromosomes and visualized by Juicebox. Relative KSHV episome tethering during reactivation for the selected region on chromosome 1 was calculated by subtracting relative 950 chimeric sequence reads in latency from relative chimeric sequence reads during reactivation. 951 Blue and red dots indicate decreased and increased chimeric sequence reads respectively. 
Fig. 5 CHD4 is both an RNA and DNA binding protein. (a) A schematic diagram of recombinant KSHVs. Mini-TurboID is fused to the $\mathrm{N}$-terminus of ORF57 open reading frame and the ORF57 binding site (MRE) of PAN RNA was mutated as shown. (b) Immunoblotting. Recombinant KSHV infected iSLK cells (TID-57 PAN-Wt and -MRE) were either untreated or induced for reactivation with doxycycline $(1 \mu \mathrm{g} / \mathrm{ml})$ and TPA $(20 \mathrm{nM})$ for $24 \mathrm{hrs}$. Blots were probed with either anti-Flag antibody (for mini-TurboID-ORF57) or Streptavidin-HRP (for biotinylated proteins). (c) Venn diagram. The Venn diagram indicates the number of identified proteins with a $P$ value less than 0.05 . Each protein ID was performed in three biological replicates. (d) PAN RNA-mediated interacting proteins with ORF57. The proteins found enriched in PAN RNAWT samples are shown. The entire list of proteins and peptide counts are presented in Supplementary Table 2. (e) siRNA screening with KSHV reactivation as readout. Recombinant KSHV reactivation, which encodes RFP under control of the PAN RNA promoter, was used to screen the effects of KSHV reactivation. RFP signal intensity was measured with Image J, and the GFP signal was used as internal controls. Three randomly selected fields in the middle of each well were quantified and the average intensity was plotted. (f) Recombinant proteins and synthesized PAN RNAs. Recombinant Flag-tagged proteins were expressed with baculovirus and purified with Flag-agarose beads. Coomassie staining of Flag-CHD4, Flag-NFkB (p65), and Flag-luciferase used for pull-down studies are shown. (g) Schematic for in vitro interaction assay performed in Fig. 5 (h-j). (h) CHD4 binds RNAs in a sequenceindependent manner. RNA pull-down was performed with the indicated biotinylated PAN RNA 973 deletions, mutation (MRE), and irrelevant RNA (luciferase mRNA), and interaction was probed 974 by immunoblotting with anti-Flag antibody. Beads alone (No RNA) was used for background 
975 control. (i) RNA pull down was performed with indicated biotinylated RNAs, and p65 and 976 Luciferase (Luc) were visualized by using anti-Flag antibody. (j) PAN RNA competes with 977 CHD4 DNA binding. Pull-down analyses with biotinylated ssRNA or dsDNA was performed. 978 CHD4 (100 nM) was incubated with biotinylated RNA (100 nM) or biotinylated dsDNA (100 nM) 979 in $40 \mu \mathrm{L}$ binding buffer. Increasing amounts of non-biotinylated PAN RNA at 1:1, 1:10, and 1:20 980 (dsDNA vs. ssRNA) were also incubated, and precipitated CHD4 protein in the pull-down was 981 probed with anti-Flag antibody. Flow-through and $10 \%$ of the input reaction before pull-down 982 were used as control.

Fig. 6 CHD4 is important for latency maintenance and establishment. (a) CHD4 overexpression, knockdown and complementation. The iSLK.219 cell line was transfected with empty vector (Vec) or mouse CHD4 cDNA or human CHD4 shRNA, or CHD4 shRNA and mouse CHD4 cDNA for $48 \mathrm{hrs}$ followed by KSHV reactivation with doxycycline $(1 \mu \mathrm{g} / \mathrm{ml})$ for 5 988 days. Encapsidated KSHV genomes in the culture supernatants were measured by qPCR to 989 assess effects of CHD4 overexpression or knockdown on KSHV reactivation. (b) CHD4 ATPase 990 activity is important for prevention of nuclear aggregate formation. iSLK cells latently 991 infected with BAC16-Wt was transfected with Flag-tagged mouse wild type CHD4, or an ATPase 992 domain mutant was transfected and stained with anti-Flag. Transcriptional factory formation was 993 assessed by staining RNAll. The percentage of aggregate formation in Flag-positive cells was 994 measured and plotted. Three biological replicates were performed, and 50 cells were counted in 995 each of the three biological replicates. (c) CHD4 ATPase activity is important for inhibiting 996 viral gene expression during reactivation. iSLK.219 cells were transduced with an equal 997 amount of CHD4 wild type (wt) and CHD4 mutant (mt) lentivirus for 2 days. Cells were 
998 reactivated with doxycycline $(1 \mu \mathrm{g} / \mathrm{ml})$ for 2 days and ORF50 and ORF57 gene expression was 999 quantified by RT-qPCR. Untreated cells were used for calculating the relative expression of 1000 ORF50 and ORF57. GAPDH was used as a negative control. ${ }^{*} \mathrm{p}<0.05,{ }^{* * *} \mathrm{p}<0.001$ (comparing 1001 with CHD4 Wt). (d-e) Single cell sequencing. iSLK.219 cells were reactivated by induction of 1002 K-Rta expression from doxycycline inducible promoter for $24 \mathrm{hrs}$. Single-cell sequencing was 1003 performed with the 10x Genomics platform. A cell suspension was prepared followed by cell 1004 partitioning and 3' cDNA synthesis on the Chromium Controller and dual-index library 1005 preparation with 10x Genomics kitted reagents. (d) tSNE. tSNE was applied to separate cell 1006 clusters and KSHV gene (d, left panel) and CHD4 (d, right panel) expressing cells were marked. 1007 (e) Negative correlation between CHD4 and viral gene expression. Cells were divided into 1008 groups based on the amount of KSHV transcripts. A table and distribution of cell numbers and 1009 amount of KSHV transcripts are shown in Supplementary Fig. 6a-b. Intervals used to separate 1010 cell groups are also listed in Supplementary Fig. 6b. A correlation was established based on 1011 CHD4 expression in different groups of KSHV transcripts. (f-h) CHD4 knockdown and KSHV 1012 Iytic gene expression during de novo infection. 293T cells were transduced with scramble 1013 shRNA (shScrm) or shCHD4 and stably selected with antibiotic. Immunoblot (f) and RT-qPCR 1014 (g) analysis of CHD4 in the 293T stable cell lines are shown. 293T stable cells were infected 1015 with rKSHV.219 for 24 hrs and total RNA was harvested at the indicated time points. (h) K-Rta 1016 and PAN RNA, was measured by RT-qPCR and normalized to GAPDH. ${ }^{*} p<0.05,{ }^{* *} p<0.01$ 1017 (comparing with shScrm). (i) Flow cytometry. 293T cells were transfected with control siRNA 1018 (siC) or CHD4 siRNA (siCHD4) for 48 hrs followed by infection with rKSHV.219 virus for 96 hrs. 1019 Flow cytometry analysis was performed to calculate the number of GFP- and RFP-positive cells 1020 in siC and siCHD4 knockdown cells. (j) KSHV latency model with ChAHP. KSHV episomes 
1021 tether at epigenetically active regions with the ChAHP complex, and TR-loaded LANA/ChAHP 1022 prevents robust viral IncRNA expression. The action of robust IncRNA expression in lytic KSHV 1023 infection functions as an enhancer for viral ORFs that are physically neighboring with IncRNA 1024 genomic regions. Stimulation of K-Rta, in part by decreased CHD4 expression, triggers robust 1025 PAN RNA expression in the presence of ORF57, which competes with CHD4 DNA binding to 1026 enhance KSHV reactivation and detach KSHV episomes from CHD4-enriched host chromosome 1027 regions. 
1031 1. Chang Y, Cesarman E, Pessin MS, Lee F, Culpepper J, Knowles DM, Moore PS. 1994. Identification of herpesvirus-like DNA sequences in AIDS-associated Kaposi's sarcoma. Science 266:1865-1869.

1033

2. Ganem D. 2010. KSHV and the pathogenesis of Kaposi sarcoma: listening to human biology and medicine. J Clin Invest 120:939-949.

3. Mesri EA, Cesarman E, Boshoff C. 2010. Kaposi's sarcoma and its associated herpesvirus. Nat Rev Cancer 10:707-719.

1036

1037 Schulz TF. 2006 . Wong EL, Damania B. 2005. Linking KSHV to human cancer. Curr Oncol Rep 7:349-356. characterization of two acquired immunodeficiency syndrome-related lymphoma cell lines (BC-1 and BC2) containing Kaposi's sarcoma-associated herpesvirus-like (KSHV) DNA sequences. Blood 86:2708-2714. lymphoproliferative diseases. Semin Cancer Biol 9:165-174.

8. Kedes DH, Lagunoff M, Renne R, Ganem D. 1997. Identification of the gene encoding the major latencyassociated nuclear antigen of the Kaposi's sarcoma-associated herpesvirus. J Clin Invest 100:2606-2610.

9. Nakamura H, Lu M, Gwack Y, Souvlis J, Zeichner SL, Jung JU. 2003. Global changes in Kaposi's sarcomaassociated virus gene expression patterns following expression of a tetracycline-inducible Rta transactivator. J Virol 77:4205-4220.

10. Lu M, Suen J, Frias C, Pfeiffer R, Tsai MH, Chuang E, Zeichner SL. 2004. Dissection of the Kaposi's sarcomaassociated herpesvirus gene expression program by using the viral DNA replication inhibitor cidofovir. J Virol 78:13637-13652.

1051

1052

11. Ellison TJ, Izumiya Y, Izumiya C, Luciw PA, Kung HJ. 2009. A comprehensive analysis of recruitment and transactivation potential of K-Rta and K-bZIP during reactivation of Kaposi's sarcoma-associated herpesvirus. Virology 387:76-88.

1054

1055

12. Ballestas ME, Kaye KM. 2011. The latency-associated nuclear antigen, a multifunctional protein central to Kaposi's sarcoma-associated herpesvirus latency. Future Microbiol 6:1399-1413.

1056

1057

1058

1059

1060

1061

1062

1063

1064

1065

1066

13. Stedman W, Deng Z, Lu F, Lieberman PM. 2004. ORC, MCM, and histone hyperacetylation at the Kaposi's
sarcoma-associated herpesvirus latent replication origin. J Virol 78:12566-12575.

14. Verma SC, Choudhuri T, Kaul R, Robertson ES. 2006. Latency-associated nuclear antigen (LANA) of Kaposi's sarcoma-associated herpesvirus interacts with origin recognition complexes at the LANA binding sequence within the terminal repeats. J Virol 80:2243-2256.

15. Hu J, Garber AC, Renne R. 2002. The latency-associated nuclear antigen of Kaposi's sarcoma-associated herpesvirus supports latent DNA replication in dividing cells. J Virol 76:11677-11687.

16. Barbera AJ, Chodaparambil JV, Kelley-Clarke B, Joukov V, Walter JC, Luger K, Kaye KM. 2006. The nucleosomal surface as a docking station for Kaposi's sarcoma herpesvirus LANA. Science 311:856-861.

17. Piolot T, Tramier M, Coppey M, Nicolas JC, Marechal V. 2001. Close but distinct regions of human herpesvirus 8 latency-associated nuclear antigen 1 are responsible for nuclear targeting and binding to human mitotic chromosomes. J Virol 75:3948-3959.

1068

18. Ottinger M, Christalla T, Nathan K, Brinkmann MM, Viejo-Borbolla A, Schulz TF. 2006. Kaposi's sarcomaassociated herpesvirus LANA-1 interacts with the short variant of BRD4 and releases cells from a BRD4and BRD2/RING3-induced G1 cell cycle arrest. J Virol 80:10772-10786. 
1074

1075

1076

1077

1078

1079

1080

1081

1082

1083

1084

1085

1086

1087

1088

1089

1090

1091

1092

1093

1094

1095

1096

1097

1098

1099

1100

1101

1102

1103

1104

1105

1106

1107

1108

1109

1110

1111

1112

1113

1114

1115

1116

1117

1118

1119

1120

1121

20. Kim KY, Huerta SB, Izumiya C, Wang DH, Martinez A, Shevchenko B, Kung HJ, Campbell M, Izumiya Y. 2013. Kaposi's sarcoma-associated herpesvirus (KSHV) latency-associated nuclear antigen regulates the KSHV epigenome by association with the histone demethylase KDM3A. J Virol 87:6782-6793.

21. Wang N, Wu R, Tang D, Kang R. 2021. The BET family in immunity and disease. Signal Transduct Target Ther 6:23.

22. You J, Srinivasan V, Denis GV, Harrington WJ, Jr., Ballestas ME, Kaye KM, Howley PM. 2006. Kaposi's sarcoma-associated herpesvirus latency-associated nuclear antigen interacts with bromodomain protein Brd4 on host mitotic chromosomes. J Virol 80:8909-8919.

23. Hu J, Yang Y, Turner PC, Jain V, Mclntyre LM, Renne R. 2014. LANA binds to multiple active viral and cellular promoters and associates with the H3K4methyltransferase hSET1 complex. PLoS Pathog 10:e1004240.

24. Lagunoff M, Ganem D. 1997. The structure and coding organization of the genomic termini of Kaposi's sarcoma-associated herpesvirus. Virology 236:147-154.

25. Domsic JF, Chen HS, Lu F, Marmorstein R, Lieberman PM. 2013. Molecular basis for oligomeric-DNA binding and episome maintenance by KSHV LANA. PLoS Pathog 9:e1003672.

26. Correia B, Cerqueira SA, Beauchemin C, Pires de Miranda M, Li S, Ponnusamy R, Rodrigues L, Schneider TR, Carrondo MA, Kaye KM, Simas JP, McVey CE. 2013. Crystal structure of the gamma-2 herpesvirus LANA DNA binding domain identifies charged surface residues which impact viral latency. PLoS Pathog 9:e1003673.

27. Kim J, Dean A. 2021. Enhancers navigate the three-dimensional genome to direct cell fate decisions. Curr Opin Struct Biol 71:101-109.

28. Campbell M, Watanabe T, Nakano K, Davis RR, Lyu Y, Tepper CG, Durbin-Johnson B, Fujimuro M, Izumiya Y. 2018. KSHV episomes reveal dynamic chromatin loop formation with domain-specific gene regulation. Nat Commun 9:49.

29. Chen CP, Lyu Y, Chuang F, Nakano K, Izumiya C, Jin D, Campbell M, Izumiya Y. 2017. Kaposi's SarcomaAssociated Herpesvirus Hijacks RNA Polymerase II To Create a Viral Transcriptional Factory. J Virol 91.

30. Naik NG, Nguyen TH, Roberts L, Fischer LT, Glickman K, Golas G, Papp B, Toth Z. 2020. Epigenetic factor siRNA screen during primary KSHV infection identifies novel host restriction factors for the lytic cycle of KSHV. PLoS Pathog 16:e1008268.

31. Strahan RC, McDowell-Sargent M, Uppal T, Purushothaman P, Verma SC. 2017. KSHV encoded ORF59 modulates histone arginine methylation of the viral genome to promote viral reactivation. PLoS Pathog 13:e1006482.

32. Rossetto CC, Pari G. 2012. KSHV PAN RNA associates with demethylases UTX and JMJD3 to activate lytic replication through a physical interaction with the virus genome. PLoS Pathog 8:e1002680.

33. Gunther T, Frohlich J, Herrde C, Ohno S, Burkhardt L, Adler H, Grundhoff A. 2019. A comparative epigenome analysis of gammaherpesviruses suggests cis-acting sequence features as critical mediators of rapid polycomb recruitment. PLoS Pathog 15:e1007838.

34. Toth Z, Maglinte DT, Lee SH, Lee HR, Wong LY, Brulois KF, Lee S, Buckley JD, Laird PW, Marquez VE, Jung JU. 2010. Epigenetic analysis of KSHV latent and lytic genomes. PLoS Pathog 6:e1001013.

35. Gunther T, Grundhoff A. 2010. The epigenetic landscape of latent Kaposi sarcoma-associated herpesvirus genomes. PLoS Pathog 6:e1000935.

36. Clarkson CT, Deeks EA, Samarista R, Mamayusupova H, Zhurkin VB, Teif VB. 2019. CTCF-dependent chromatin boundaries formed by asymmetric nucleosome arrays with decreased linker length. Nucleic Acids Res 47:11181-11196.

37. Barisic D, Stadler MB, lurlaro M, Schubeler D. 2019. Mammalian ISWI and SWI/SNF selectively mediate binding of distinct transcription factors. Nature 569:136-140.

38. Goodman JV, Yamada T, Yang Y, Kong L, Wu DY, Zhao G, Gabel HW, Bonni A. 2020. The chromatin remodeling enzyme Chd4 regulates genome architecture in the mouse brain. Nat Commun 11:3419. 
39. Ostapcuk V, Mohn F, Carl SH, Basters A, Hess D, lesmantavicius V, Lampersberger L, Flemr M, Pandey A, Thoma NH, Betschinger J, Buhler M. 2018. Activity-dependent neuroprotective protein recruits HP1 and CHD4 to control lineage-specifying genes. Nature 557:739-743.

40. Sun X, Yu W, Li L, Sun Y. 2020. ADNP Controls Gene Expression Through Local Chromatin Architecture by Association With BRG1 and CHD4. Front Cell Dev Biol 8:553.

41. Arends T, Dege C, Bortnick A, Danhorn T, Knapp JR, Jia H, Harmacek L, Fleenor CJ, Straign D, Walton K, Leach SM, Feeney AJ, Murre C, O'Connor BP, Hagman JR. 2019. CHD4 is essential for transcriptional repression and lineage progression in B lymphopoiesis. Proc Natl Acad Sci U S A 116:10927-10936.

42. Farnung L, Ochmann M, Cramer P. 2020. Nucleosome-CHD4 chromatin remodeler structure maps human disease mutations. Elife 9.

43. Kaaij LT, Mohn F, van der Weide RH, de Wit E, Buhler M. 2019. The ChAHP Complex Counteracts Chromatin Looping at CTCF Sites that Emerged from SINE Expansions in Mouse. Cell 178:1437-1451 e1414.

44. Zhao H, Han Z, Liu X, Gu J, Tang F, Wei G, Jin Y. 2017. The chromatin remodeler Chd4 maintains embryonic stem cell identity by controlling pluripotency- and differentiation-associated genes. J Biol Chem 292:85078519.

45. Wang HC, Chou CL, Yang CC, Huang WL, Hsu YC, Luo CW, Chen TJ, Li CF, Pan MR. 2019. Over-Expression of CHD4 Is an Independent Biomarker of Poor Prognosis in Patients with Rectal Cancers Receiving Concurrent Chemoradiotherapy. Int J Mol Sci 20.

46. Xia L, Huang W, Bellani M, Seidman MM, Wu K, Fan D, Nie Y, Cai Y, Zhang YW, Yu LR, Li H, Zahnow CA, Xie W, Chiu Yen RW, Rassool FV, Baylin SB. 2017. CHD4 Has Oncogenic Functions in Initiating and Maintaining Epigenetic Suppression of Multiple Tumor Suppressor Genes. Cancer Cell 31:653-668 e657.

47. Kovac K, Sauer A, Macinkovic I, Awe S, Finkernagel F, Hoffmeister H, Fuchs A, Muller R, Rathke C, Langst G, Brehm A. 2018. Tumour-associated missense mutations in the dMi-2 ATPase alters nucleosome remodelling properties in a mutation-specific manner. Nat Commun 9:2112.

48. Weiss K, Terhal PA, Cohen L, Bruccoleri M, Irving M, Martinez AF, Rosenfeld JA, Machol K, Yang Y, Liu P, Walkiewicz M, Beuten J, Gomez-Ospina N, Haude K, Fong CT, Enns GM, Bernstein JA, Fan J, Gotway G, Ghorbani M, Study DDD, van Gassen K, Monroe GR, van Haaften G, Basel-Vanagaite L, Yang XJ, Campeau PM, Muenke M. 2016. De Novo Mutations in CHD4, an ATP-Dependent Chromatin Remodeler Gene, Cause an Intellectual Disability Syndrome with Distinctive Dysmorphisms. Am J Hum Genet 99:934-941.

49. Sartorelli V, Lauberth SM. 2020. Enhancer RNAs are an important regulatory layer of the epigenome. Nat Struct Mol Biol 27:521-528.

50. Bose DA, Donahue G, Reinberg D, Shiekhattar R, Bonasio R, Berger SL. 2017. RNA Binding to CBP Stimulates Histone Acetylation and Transcription. Cell 168:135-149 e122.

51. Hah N, Murakami S, Nagari A, Danko CG, Kraus WL. 2013. Enhancer transcripts mark active estrogen receptor binding sites. Genome Res 23:1210-1223.

52. Rahnamoun H, Lee J, Sun Z, Lu H, Ramsey KM, Komives EA, Lauberth SM. 2018. RNAs interact with BRD4 to promote enhanced chromatin engagement and transcription activation. Nat Struct Mol Biol 25:687697.

53. Morf J, Basu S, Amaral PP. 2020. RNA, Genome Output and Input. Front Genet 11:589413.

54. Song MJ, Brown HJ, Wu TT, Sun R. 2001. Transcription activation of polyadenylated nuclear rna by rta in human herpesvirus 8/Kaposi's sarcoma-associated herpesvirus. J Virol 75:3129-3140.

55. Sun R, Lin SF, Gradoville L, Miller G. 1996. Polyadenylylated nuclear RNA encoded by Kaposi sarcomaassociated herpesvirus. Proc Natl Acad Sci U S A 93:11883-11888.

56. Conrad NK, Steitz JA. 2005. A Kaposi's sarcoma virus RNA element that increases the nuclear abundance of intronless transcripts. EMBO J 24:1831-1841.

57. Massimelli MJ, Kang JG, Majerciak V, Le SY, Liewehr DJ, Steinberg SM, Zheng ZM. 2011. Stability of a long noncoding viral RNA depends on a 9-nt core element at the RNA 5' end to interact with viral ORF57 and cellular PABPC1. Int J Biol Sci 7:1145-1160. 
58. Mitton-Fry RM, DeGregorio SJ, Wang J, Steitz TA, Steitz JA. 2010. Poly(A) tail recognition by a viral RNA element through assembly of a triple helix. Science 330:1244-1247.

59. Massimelli MJ, Majerciak V, Kruhlak M, Zheng ZM. 2013. Interplay between polyadenylate-binding protein 1 and Kaposi's sarcoma-associated herpesvirus ORF57 in accumulation of polyadenylated nuclear RNA, a viral long noncoding RNA. J Virol 87:243-256.

60. Rossetto CC, Tarrant-Elorza M, Verma S, Purushothaman P, Pari GS. 2013. Regulation of viral and cellular gene expression by Kaposi's sarcoma-associated herpesvirus polyadenylated nuclear RNA. J Virol 87:55405553.

61. Campbell M, Kim KY, Chang PC, Huerta S, Shevchenko B, Wang DH, Izumiya C, Kung HJ, Izumiya Y. 2014. A lytic viral long noncoding RNA modulates the function of a latent protein. J Virol 88:1843-1848.

62. Withers JB, Li ES, Vallery TK, Yario TA, Steitz JA. 2018. Two herpesviral noncoding PAN RNAs are functionally homologous but do not associate with common chromatin loci. PLoS Pathog 14:e1007389.

63. Xiao B, Verma SC, Cai Q, Kaul R, Lu J, Saha A, Robertson ES. 2010. Bub1 and CENP-F can contribute to Kaposi's sarcoma-associated herpesvirus genome persistence by targeting LANA to kinetochores. J Virol 84:9718-9732.

64. Branon TC, Bosch JA, Sanchez AD, Udeshi ND, Svinkina T, Carr SA, Feldman JL, Perrimon N, Ting AY. 2018. Efficient proximity labeling in living cells and organisms with TurbolD. Nat Biotechnol 36:880-887.

65. Kumar A, Salemi M, Bhullar R, Guevara-Plunkett S, Lyu Y, Wang KH, Izumiya C, Campbell M, Nakajima KI, Izumiya Y. 2021. Proximity Biotin Labeling Reveals KSHV Interferon Regulatory Factor Networks. J Virol doi:10.1128/JVI.02049-20.

66. Hellert J, Weidner-Glunde M, Krausze J, Richter U, Adler H, Fedorov R, Pietrek M, Ruckert J, Ritter C, Schulz TF, Luhrs T. 2013. A structural basis for BRD2/4-mediated host chromatin interaction and oligomer assembly of Kaposi sarcoma-associated herpesvirus and murine gammaherpesvirus LANA proteins. PLoS Pathog 9:e1003640.

67. Verma SC, Cai Q, Kreider E, Lu J, Robertson ES. 2013. Comprehensive analysis of LANA interacting proteins essential for viral genome tethering and persistence. PLoS One 8:e74662.

68. Si H, Verma SC, Lampson MA, Cai Q, Robertson ES. 2008. Kaposi's sarcoma-associated herpesvirusencoded LANA can interact with the nuclear mitotic apparatus protein to regulate genome maintenance and segregation. J Virol 82:6734-6746.

69. Lim C, Lee D, Seo T, Choi C, Choe J. 2003. Latency-associated nuclear antigen of Kaposi's sarcomaassociated herpesvirus functionally interacts with heterochromatin protein 1. J Biol Chem 278:7397-7405.

70. Skene PJ, Henikoff S. 2017. An efficient targeted nuclease strategy for high-resolution mapping of DNA binding sites. Elife 6.

71. Manzano M, Gunther T, Ju H, Nicholas J, Bartom ET, Grundhoff A, Gottwein E. 2020. Kaposi's SarcomaAssociated Herpesvirus Drives a Super-Enhancer-Mediated Survival Gene Expression Program in Primary Effusion Lymphoma. mBio 11.

72. Wang C, Zhang L, Ke L, Ding W, Jiang S, Li D, Narita Y, Hou I, Liang J, Li S, Xiao H, Gottwein E, Kaye KM, Teng M, Zhao B. 2020. Primary effusion lymphoma enhancer connectome links super-enhancers to dependency factors. Nat Commun 11:6318.

73. Garber AC, Shu MA, Hu J, Renne R. 2001. DNA binding and modulation of gene expression by the latencyassociated nuclear antigen of Kaposi's sarcoma-associated herpesvirus. J Virol 75:7882-7892.

74. Purushothaman P, Thakker S, Verma SC. 2015. Transcriptome analysis of Kaposi's sarcoma-associated herpesvirus during de novo primary infection of human B and endothelial cells. J Virol 89:3093-3111.

75. Arnold PR, Wells AD, Li XC. 2019. Diversity and Emerging Roles of Enhancer RNA in Regulation of Gene Expression and Cell Fate. Front Cell Dev Biol 7:377.

76. Morra R, Lee BM, Shaw H, Tuma R, Mancini EJ. 2012. Concerted action of the PHD, chromo and motor domains regulates the human chromatin remodelling ATPase CHD4. FEBS Lett 586:2513-2521. 
77. Weiss K, Lazar HP, Kurolap A, Martinez AF, Paperna T, Cohen L, Smeland MF, Whalen S, Heide S, Keren B, Terhal P, Irving M, Takaku M, Roberts JD, Petrovich RM, Schrier Vergano SA, Kenney A, Hove H, DeChene E, Quinonez SC, Colin E, Ziegler A, Rumple M, Jain M, Monteil D, Roeder ER, Nugent K, van Haeringen A, Gambello M, Santani A, Medne L, Krock B, Skraban CM, Zackai EH, Dubbs HA, Smol T, Ghoumid J, Parker MJ, Wright M, Turnpenny P, Clayton-Smith J, Metcalfe K, Kurumizaka H, Gelb BD, Baris Feldman H, Campeau PM, Muenke M, Wade PA, Lachlan K. 2020. The CHD4-related syndrome: a comprehensive investigation of the clinical spectrum, genotype-phenotype correlations, and molecular basis. Genet Med 22:389-397.

78. Hansen AS, Hsieh TS, Cattoglio C, Pustova I, Saldana-Meyer R, Reinberg D, Darzacq X, Tjian R. 2019. Distinct Classes of Chromatin Loops Revealed by Deletion of an RNA-Binding Region in CTCF. Mol Cell 76:395-411 e313.

79. Saldana-Meyer R, Rodriguez-Hernaez J, Escobar T, Nishana M, Jacome-Lopez K, Nora EP, Bruneau BG, Tsirigos A, Furlan-Magaril M, Skok J, Reinberg D. 2019. RNA Interactions Are Essential for CTCF-Mediated Genome Organization. Mol Cell 76:412-422 e415.

80. Nair SJ, Yang L, Meluzzi D, Oh S, Yang F, Friedman MJ, Wang S, Suter T, Alshareedah I, Gamliel A, Ma Q, Zhang J, Hu Y, Tan Y, Ohgi KA, Jayani RS, Banerjee PR, Aggarwal AK, Rosenfeld MG. 2019. Phase separation of ligand-activated enhancers licenses cooperative chromosomal enhancer assembly. Nat Struct Mol Biol 26:193-203.

81. Isoda T, Moore AJ, He Z, Chandra V, Aida M, Denholtz M, Piet van Hamburg J, Fisch KM, Chang AN, FahI SP, Wiest DL, Murre C. 2017. Non-coding Transcription Instructs Chromatin Folding and Compartmentalization to Dictate Enhancer-Promoter Communication and T Cell Fate. Cell 171:103-119 e118.

82. Coe BP, Stessman HAF, Sulovari A, Geisheker MR, Bakken TE, Lake AM, Dougherty JD, Lein ES, Hormozdiari F, Bernier RA, Eichler EE. 2019. Neurodevelopmental disease genes implicated by de novo mutation and copy number variation morbidity. Nat Genet 51:106-116.

83. Sifrim A, Hitz MP, Wilsdon A, Breckpot J, Turki SH, Thienpont B, McRae J, Fitzgerald TW, Singh T, Swaminathan GJ, Prigmore E, Rajan D, Abdul-Khaliq H, Banka S, Bauer UM, Bentham J, Berger F, Bhattacharya S, Bu'Lock F, Canham N, Colgiu IG, Cosgrove C, Cox H, Daehnert I, Daly A, Danesh J, Fryer A, Gewillig M, Hobson E, Hoff K, Homfray T, Study I, Kahlert AK, Ketley A, Kramer HH, Lachlan K, Lampe AK, Louw JJ, Manickara AK, Manase D, McCarthy KP, Metcalfe K, Moore C, Newbury-Ecob R, Omer SO, Ouwehand WH, Park SM, Parker MJ, Pickardt T, Pollard MO, et al. 2016. Distinct genetic architectures for syndromic and nonsyndromic congenital heart defects identified by exome sequencing. Nat Genet 48:1060-1065.

84. Zhao S, Choi M, Overton JD, Bellone S, Roque DM, Cocco E, Guzzo F, English DP, Varughese J, Gasparrini S, Bortolomai I, Buza N, Hui P, Abu-Khalaf M, Ravaggi A, Bignotti E, Bandiera E, Romani C, Todeschini P, Tassi R, Zanotti L, Carrara L, Pecorelli S, Silasi DA, Ratner E, Azodi M, Schwartz PE, Rutherford TJ, Stiegler AL, Mane S, Boggon TJ, Schlessinger J, Lifton RP, Santin AD. 2013. Landscape of somatic single-nucleotide and copy-number mutations in uterine serous carcinoma. Proc Natl Acad Sci U S A 110:2916-2921.

85. Le Gallo M, O'Hara AJ, Rudd ML, Urick ME, Hansen NF, O'Neil NJ, Price JC, Zhang S, England BM, Godwin AK, Sgroi DC, Program NIHISCCS, Hieter P, Mullikin JC, Merino MJ, Bell DW. 2012. Exome sequencing of serous endometrial tumors identifies recurrent somatic mutations in chromatin-remodeling and ubiquitin ligase complex genes. Nat Genet 44:1310-1315.

86. Kim KD, Tanizawa H, De Leo A, Vladimirova O, Kossenkov A, Lu F, Showe LC, Noma KI, Lieberman PM. 2020. Epigenetic specifications of host chromosome docking sites for latent Epstein-Barr virus. Nat Commun 11:877.

87. Carbone A, Gloghini A, Cozzi MR, Capello D, Steffan A, Monini P, De Marco L, Gaidano G. 2000. Expression of MUM1/IRF4 selectively clusters with primary effusion lymphoma among lymphomatous effusions: implications for disease histogenesis and pathogenesis. $\mathrm{Br} J$ Haematol 111:247-257. 
88. Majerciak V, Pripuzova N, McCoy JP, Gao SJ, Zheng ZM. 2007. Targeted disruption of Kaposi's sarcomaassociated herpesvirus ORF57 in the viral genome is detrimental for the expression of ORF59, K8alpha, and K8.1 and the production of infectious virus. J Virol 81:1062-1071.

89. Rao SS, Huntley MH, Durand NC, Stamenova EK, Bochkov ID, Robinson JT, Sanborn AL, Machol I, Omer AD, Lander ES, Aiden EL. 2014. A 3D map of the human genome at kilobase resolution reveals principles of chromatin looping. Cell 159:1665-1680.

90. Belaghzal H, Dekker J, Gibcus JH. 2017. Hi-C 2.0: An optimized Hi-C procedure for high-resolution genomewide mapping of chromosome conformation. Methods 123:56-65.

91. Schmitt AD, Hu M, Ren B. 2016. Genome-wide mapping and analysis of chromosome architecture. Nature Reviews Molecular Cell Biology 17:743-755.

92. Schoenfelder S, Javierre B-M, Furlan-Magaril M, Wingett SW, Fraser P. 2018. Promoter Capture Hi-C: High-resolution, Genome-wide Profiling of Promoter Interactions. Journal of Visualized Experiments : JoVE doi:10.3791/57320.

93. Jung I, Schmitt A, Diao Y, Lee AJ, Liu T, Yang D, Tan C, Eom J, Chan M, Chee S, Chiang Z, Kim C, Masliah E, Barr CL, Li B, Kuan S, Kim D, Ren B. 2019. A compendium of promoter-centered long-range chromatin interactions in the human genome. Nat Genet 51:1442-1449.

94. Servant N, Varoquaux N, Lajoie BR, Viara E, Chen CJ, Vert JP, Heard E, Dekker J, Barillot E. 2015. HiC-Pro: an optimized and flexible pipeline for Hi-C data processing. Genome Biol 16:259.

95. Khan A, Mathelier A. 2017. Intervene: a tool for intersection and visualization of multiple gene or genomic region sets. BMC Bioinformatics 18:287.

96. Wingett S, Ewels P, Furlan-Magaril M, Nagano T, Schoenfelder S, Fraser P, Andrews S. 2015. HiCUP: pipeline for mapping and processing Hi-C data. F1000Res 4:1310.

97. Durand NC, Robinson JT, Shamim MS, Machol I, Mesirov JP, Lander ES, Aiden EL. 2016. Juicebox Provides a Visualization System for Hi-C Contact Maps with Unlimited Zoom. Cell Syst 3:99-101.

98. Heinz S, Benner C, Spann N, Bertolino E, Lin YC, Laslo P, Cheng JX, Murre C, Singh H, Glass CK. 2010. Simple combinations of lineage-determining transcription factors prime cis-regulatory elements required for macrophage and B cell identities. Mol Cell 38:576-589.

99. Langmead B, Salzberg SL. 2012. Fast gapped-read alignment with Bowtie 2. Nat Methods 9:357-359.

100. Kim D, Paggi JM, Park C, Bennett C, Salzberg SL. 2019. Graph-based genome alignment and genotyping with HISAT2 and HISAT-genotype. Nat Biotechnol 37:907-915.

101. Zhang Y, Liu T, Meyer CA, Eeckhoute J, Johnson DS, Bernstein BE, Nusbaum C, Myers RM, Brown M, Li W, Liu XS. 2008. Model-based analysis of ChIP-Seq (MACS). Genome Biol 9:R137.

102. Freese NH, Norris DC, Loraine AE. 2016. Integrated genome browser: visual analytics platform for genomics. Bioinformatics 32:2089-2095.

103. Shen L, Shao N, Liu X, Nestler E. 2014. ngs.plot: Quick mining and visualization of next-generation sequencing data by integrating genomic databases. BMC Genomics 15:284.

104. Stuart T, Butler A, Hoffman P, Hafemeister C, Papalexi E, Mauck WM, 3rd, Hao Y, Stoeckius M, Smibert P, Satija R. 2019. Comprehensive Integration of Single-Cell Data. Cell 177:1888-1902 e1821.

105. Izumiya Y, Izumiya C, Hsia D, Ellison TJ, Luciw PA, Kung HJ. 2009. NF-kappaB serves as a cellular sensor of Kaposi's sarcoma-associated herpesvirus latency and negatively regulates K-Rta by antagonizing the RBP-Jkappa coactivator. J Virol 83:4435-4446.

106. Izumiya Y, Ellison TJ, Yeh ET, Jung JU, Luciw PA, Kung HJ. 2005. Kaposi's sarcoma-associated herpesvirus K-bZIP represses gene transcription via SUMO modification. J Virol 79:9912-9925.

107. Tischer BK, Smith GA, Osterrieder N. 2010. En passant mutagenesis: a two step markerless red recombination system. Methods Mol Biol 634:421-430.

108. Brulois KF, Chang H, Lee AS, Ensser A, Wong LY, Toth Z, Lee SH, Lee HR, Myoung J, Ganem D, Oh TK, Kim JF, Gao SJ, Jung JU. 2012. Construction and manipulation of a new Kaposi's sarcoma-associated herpesvirus bacterial artificial chromosome clone. J Virol 86:9708-9720. 
1313 109. Fakhari FD, Dittmer DP. 2002. Charting latency transcripts in Kaposi's sarcoma-associated herpesvirus by whole-genome real-time quantitative PCR. J Virol 76:6213-6223.

1315 110. Nesvizhskii Al, Keller A, Kolker E, Aebersold R. 2003. A statistical model for identifying proteins by tandem 1316 mass spectrometry. Anal Chem 75:4646-4658.

111. Zhou Y, Zhou B, Pache L, Chang M, Khodabakhshi AH, Tanaseichuk O, Benner C, Chanda SK. 2019. Metascape provides a biologist-oriented resource for the analysis of systems-level datasets. Nat Commun 10:1523. 
a

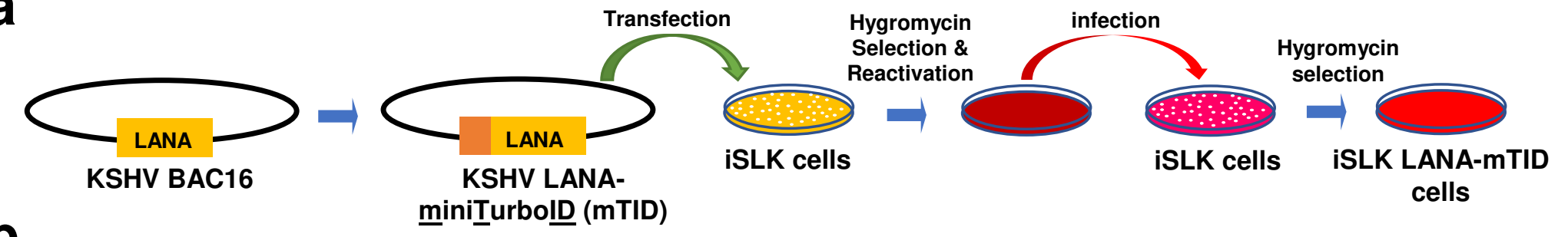

b miniTurbolD $(m$ TID)

Biotinylation Stop Rxn Lysis $\underline{I P} \quad \underline{\text { Mass-Spec }}$ Incubation $\longrightarrow$ Incubation $\longrightarrow$ Protein $\longrightarrow$ Strep- $\rightarrow$ Peptide $\left(37^{\circ} \mathrm{C}, 1 \mathrm{~h}\right) \longrightarrow\left(4^{\circ} \mathrm{C}, 1 \mathrm{~h}\right) \longrightarrow$ extraction $\rightarrow$ Biotin IP $\rightarrow$ Quantification

C

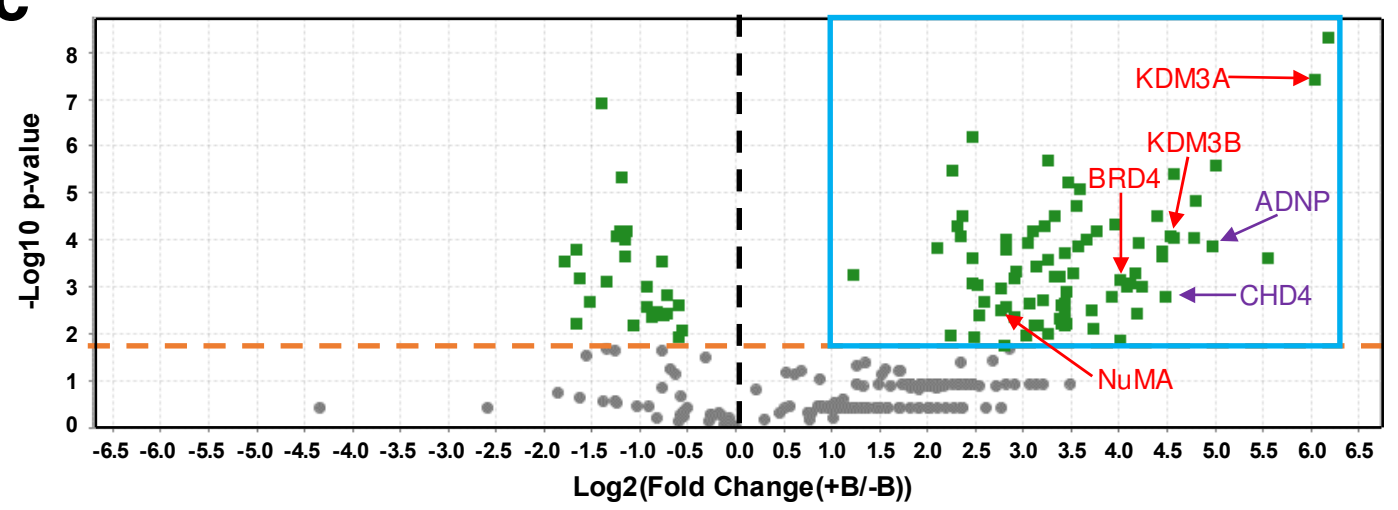

Log2 (Fold Change(+B/-B))

d
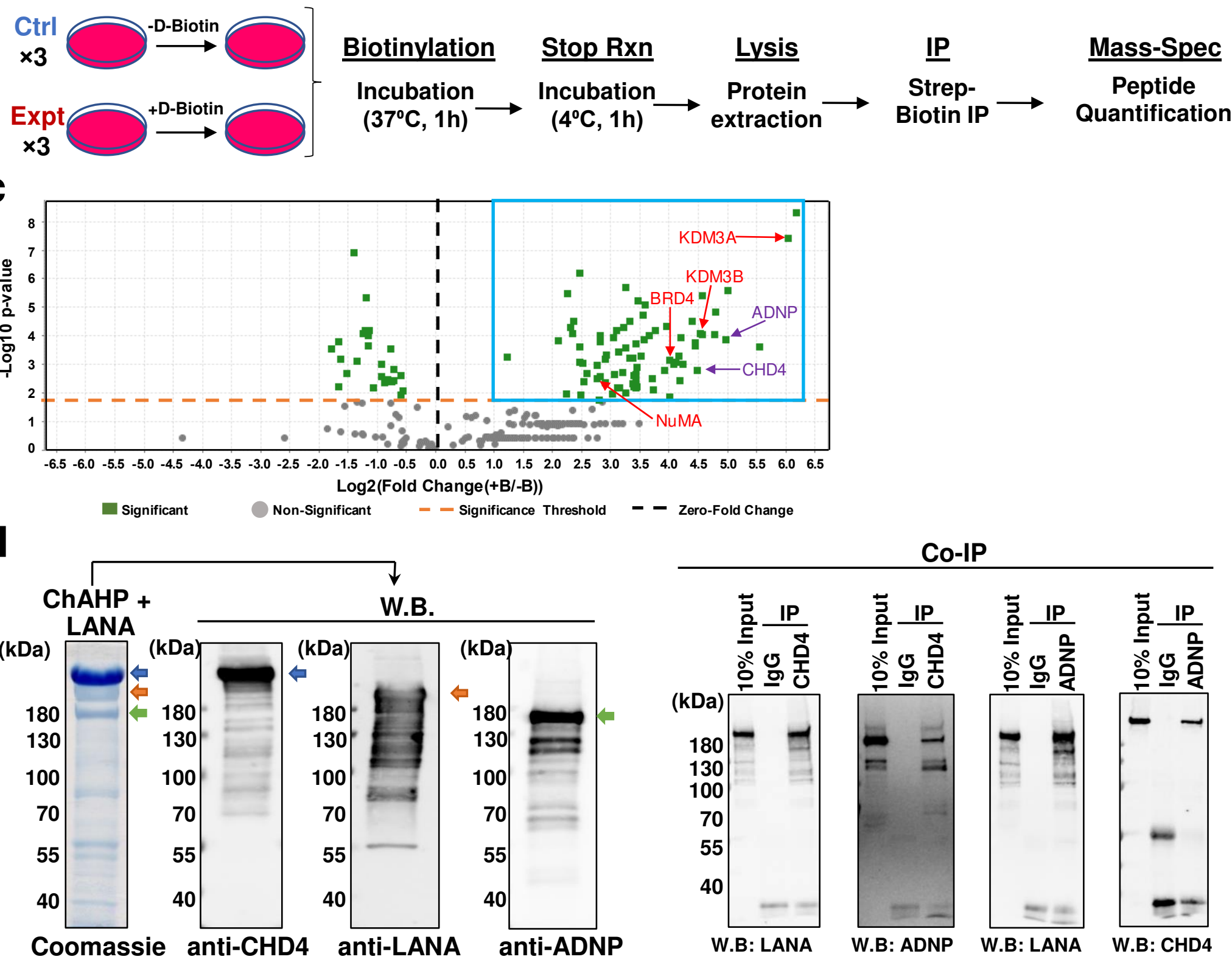

e
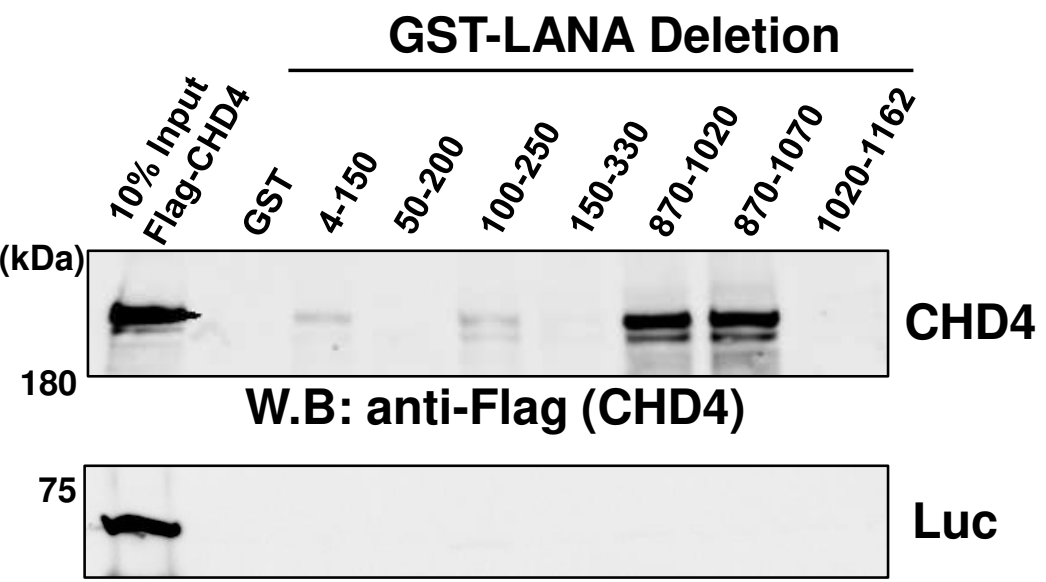

Luc

W.B: anti-Flag (Luciferase)

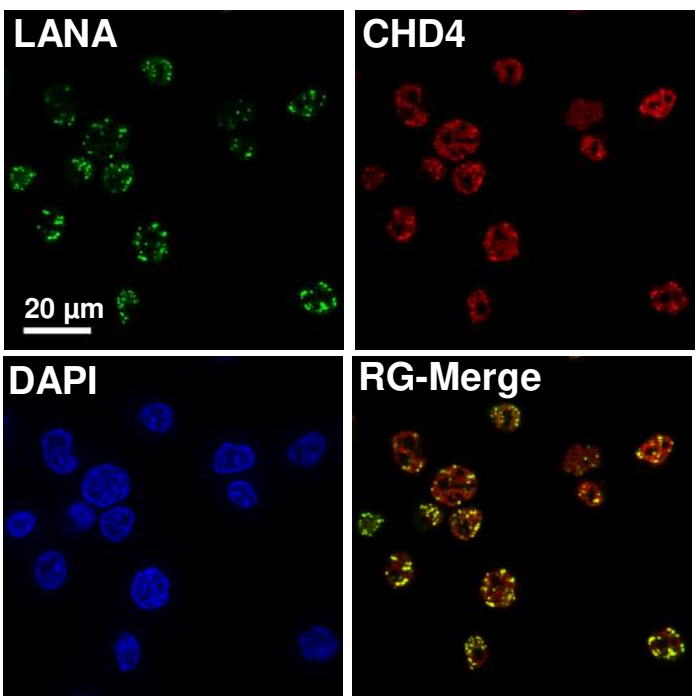

Fig. 2 


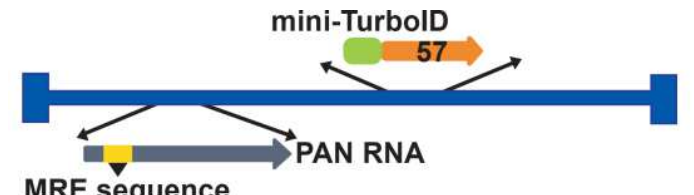

MRE sequence

PAN RNA Wt : 28701- ACCTATGGATTTTG- 28714

PAN RAN Mut : 28701- ACCCTGCACGTCTG- 28714
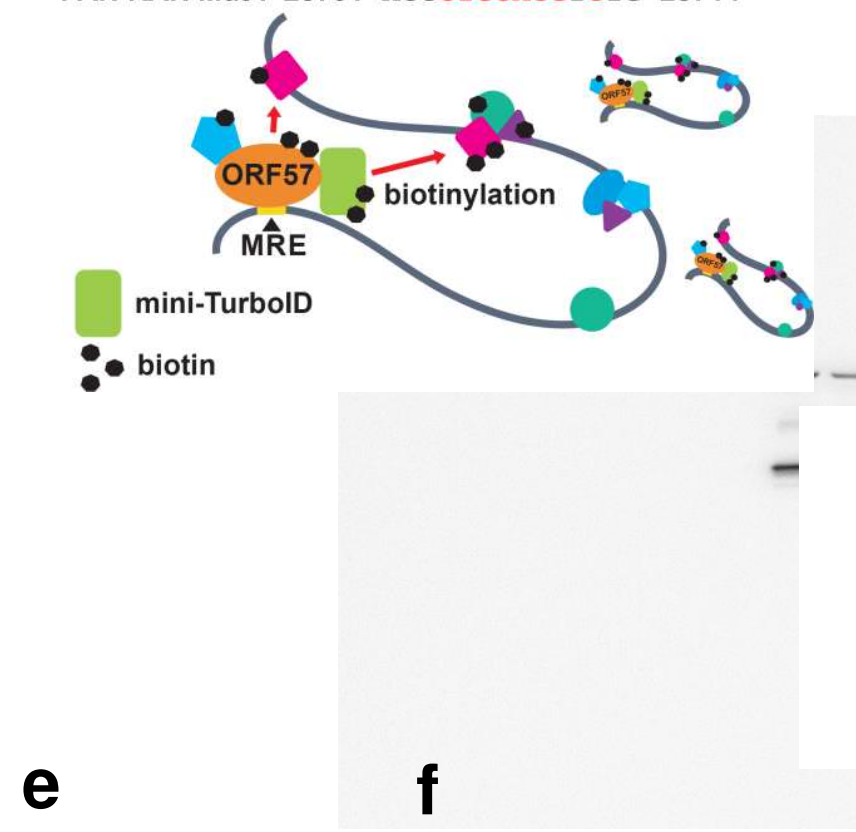

Flag-tagged Recombinant Proteins

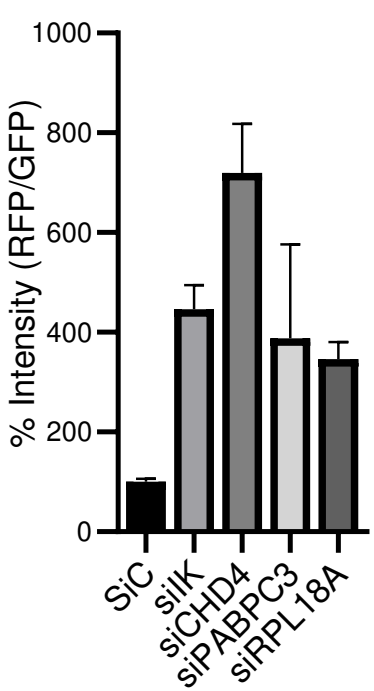

h

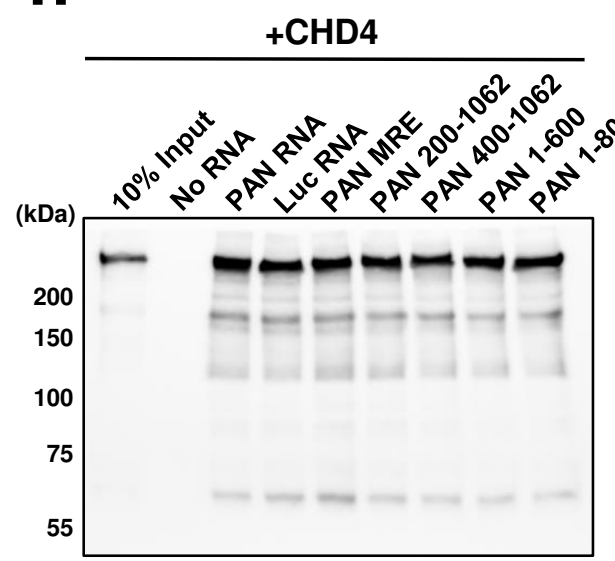

W.B: anti-Flag (CHD4)

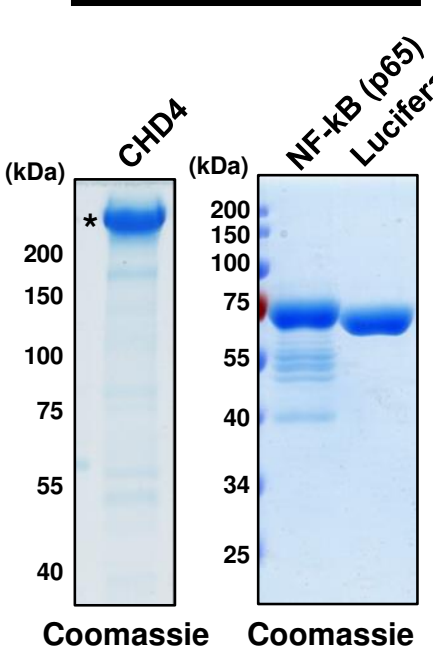

b
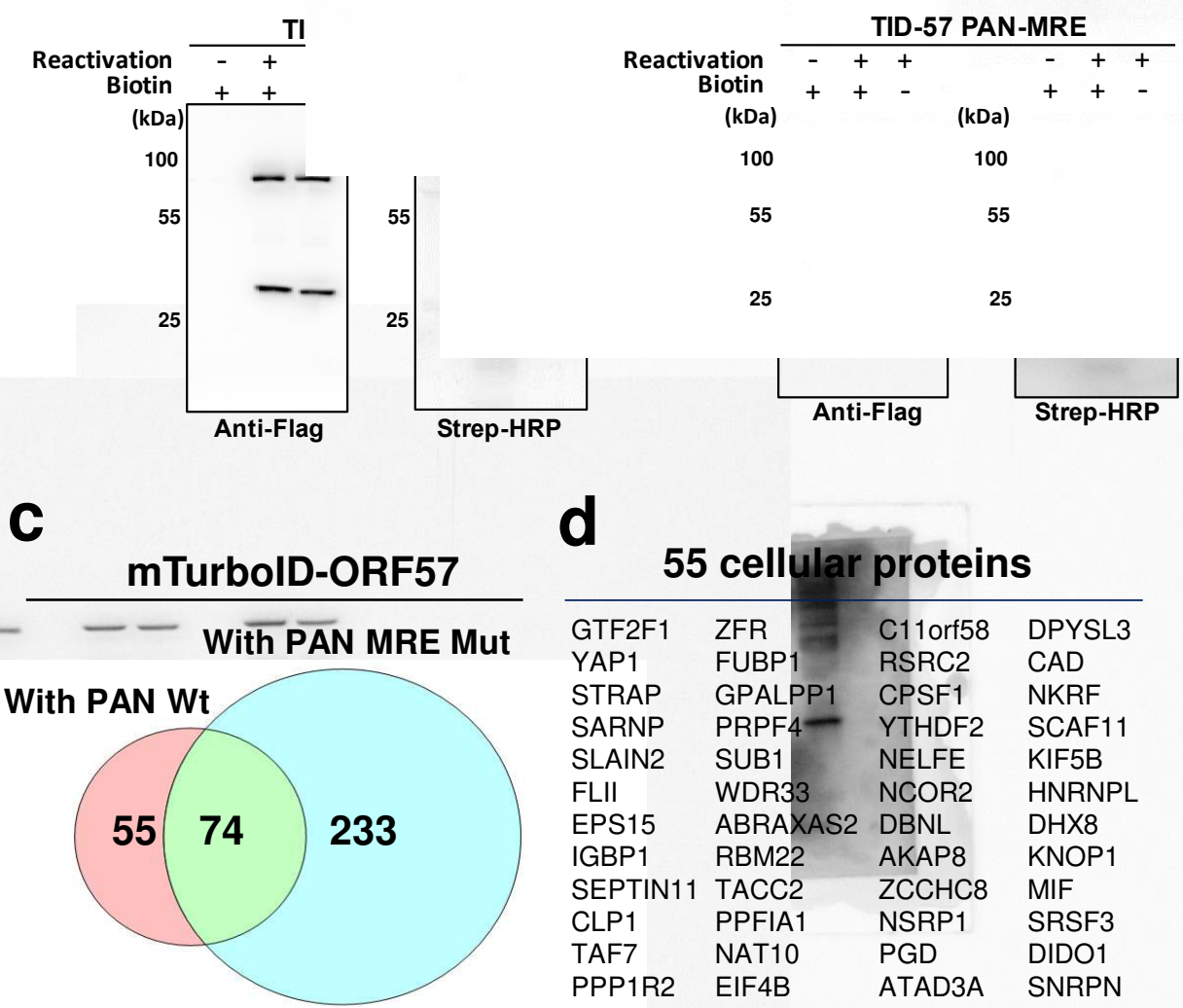

d

55 cellular proteins

\begin{tabular}{llll}
\hline GTF2F1 & ZFR & C11orf58 & DPYSL3 \\
YAP1 & FUBP1 & RSRC2 & CAD \\
STRAP & GPALPP1 & CPSF1 & NKRF \\
SARNP & PRPF4 & YTHDF2 & SCAF11 \\
SLAIN2 & SUB1 & NELFE & KIF5B \\
FLII & WDR33 & NCOR2 & HNRNPL \\
EPS15 & ABRAXAS2 & DBNL & DHX8 \\
IGBP1 & RBM22 & AKAP8 & KNOP1 \\
SEPTIN11 & TACC2 & ZCCHC8 & MIF \\
CLP1 & PPFIA1 & NSRP1 & SRSF3 \\
TAF7 & NAT10 & PGD & DIDO1 \\
PPP1R2 & EIF4B & ATAD3A & SNRPN \\
SLU7 & ELOA & CHD4 & MFAP1 \\
SF1 & GPATCH1 & NCL &
\end{tabular}

Biotin labelled

RNAs

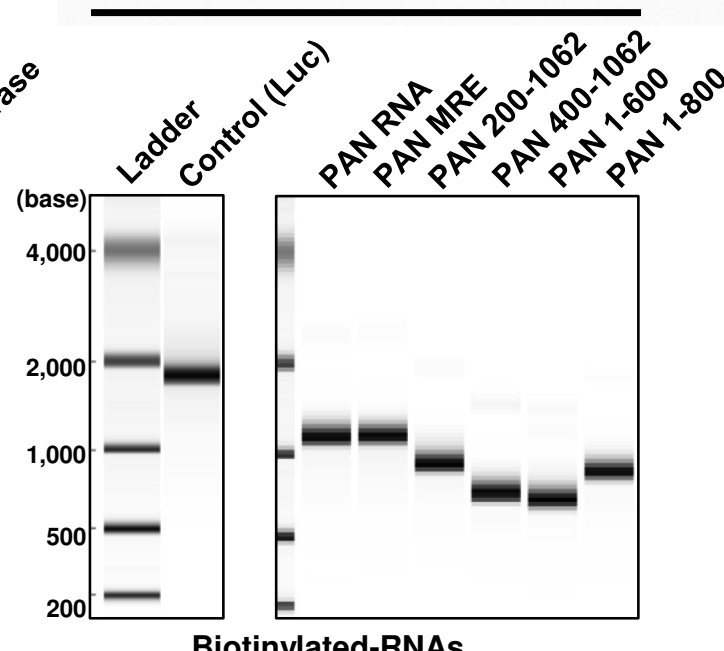

9

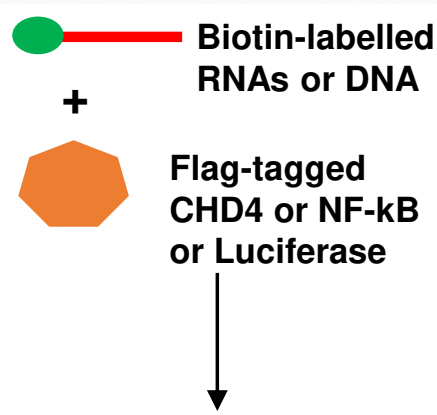

IP: RNAs or DNA (Strep beads)

WB: anti-Flag (CHD4/NF$k B /$ Luciferase)

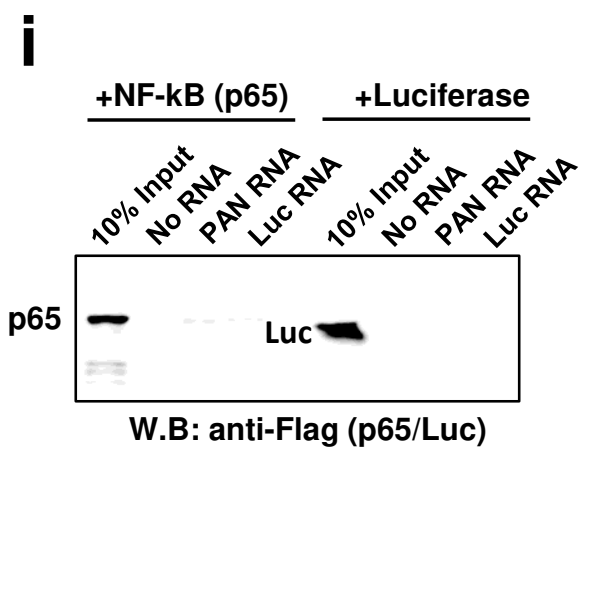

W.B: anti-Flag (p65/Luc)

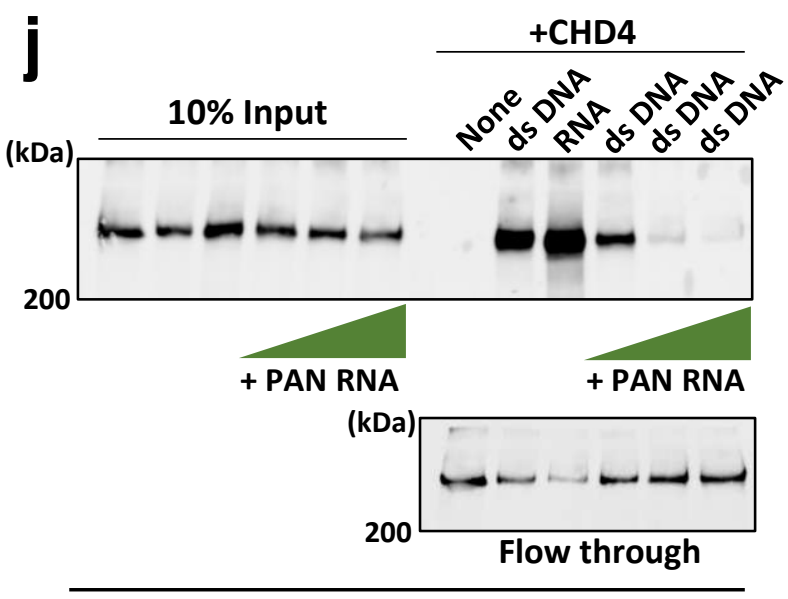

W.B: anti-Flag (CHD4) 
a $\quad$ b

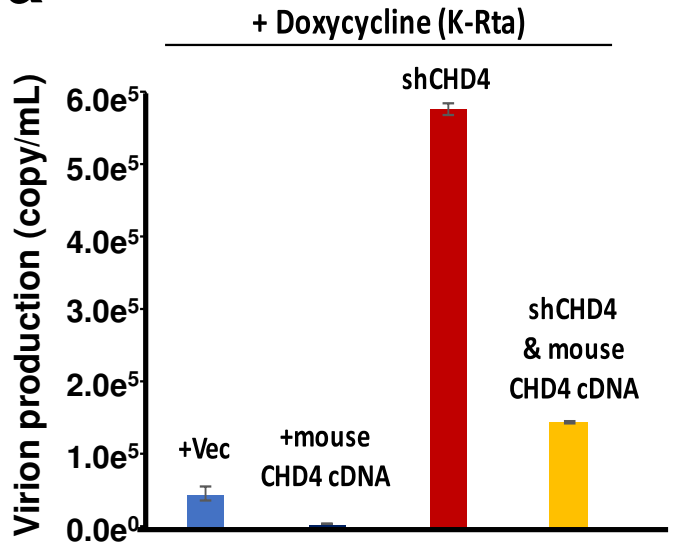

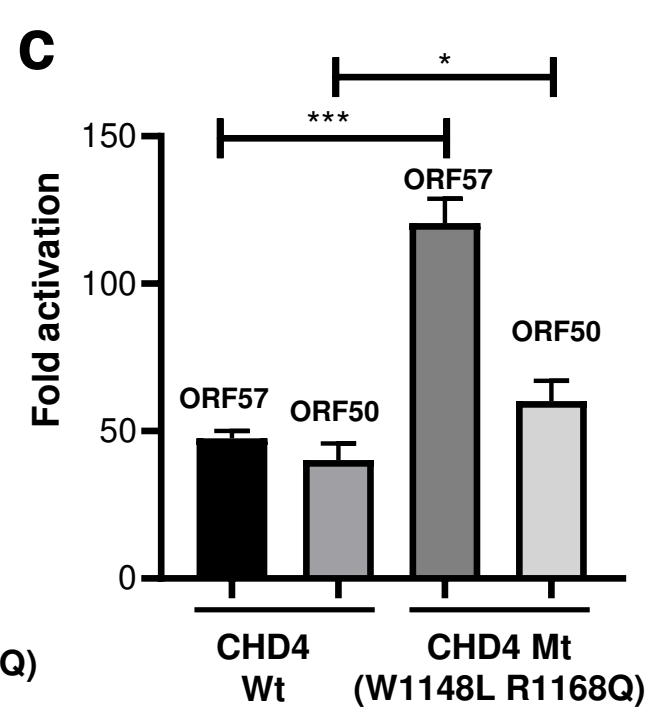

d

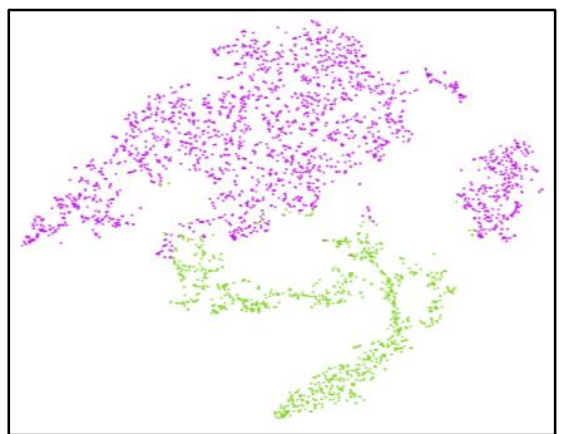

KSHV expression (-)

KSHV expression (+)

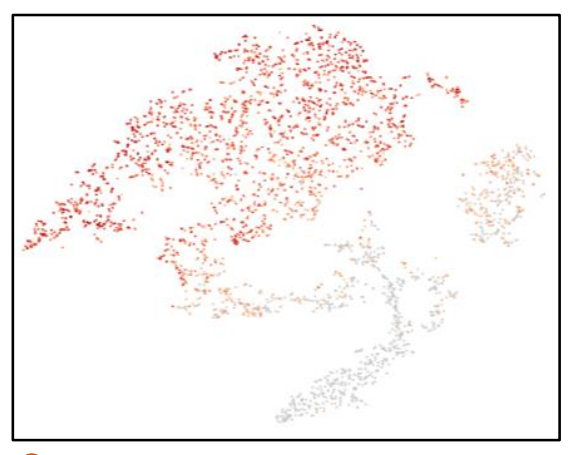

CHD4 (+)

CHD4 (-)

e
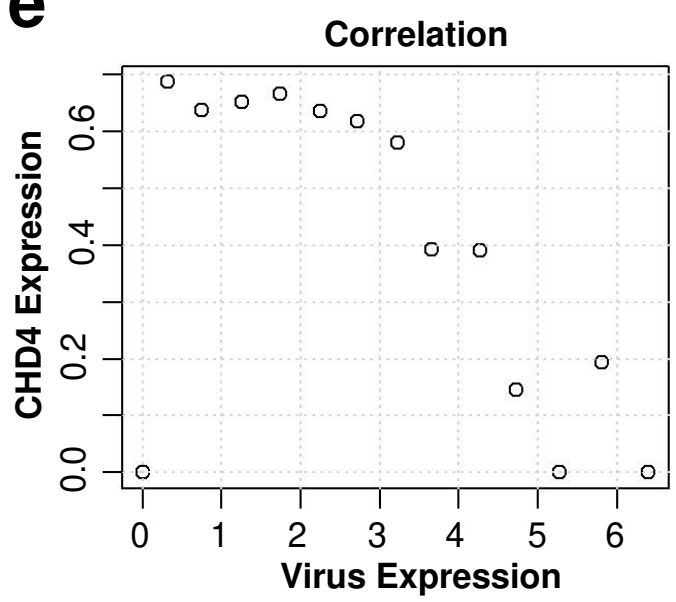

f

g

CHD4
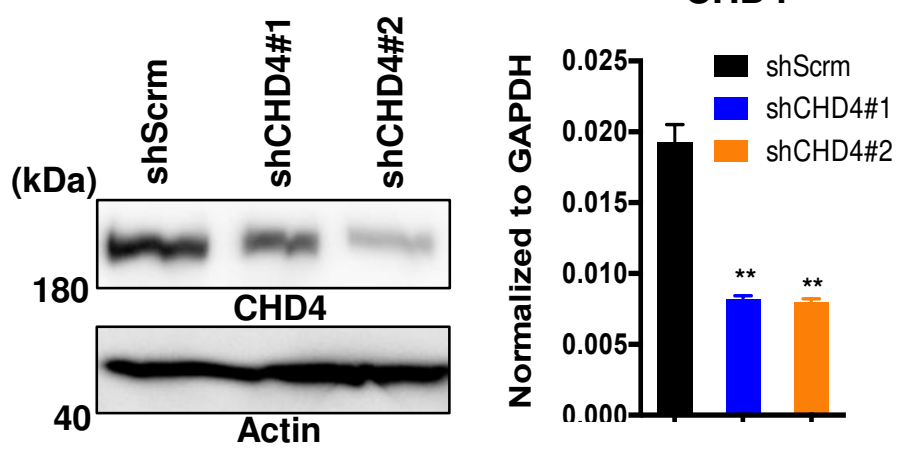

h

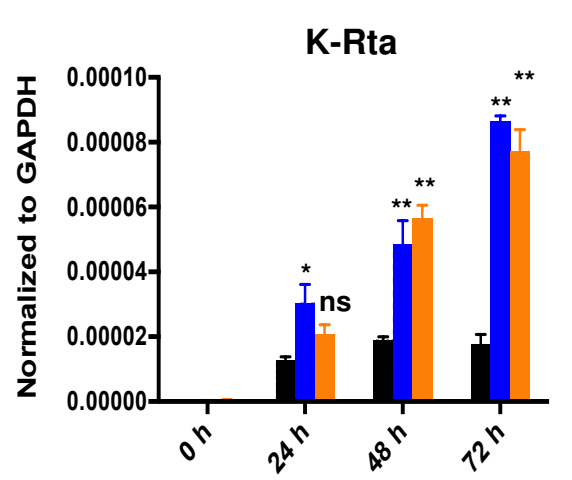

PAN RNA

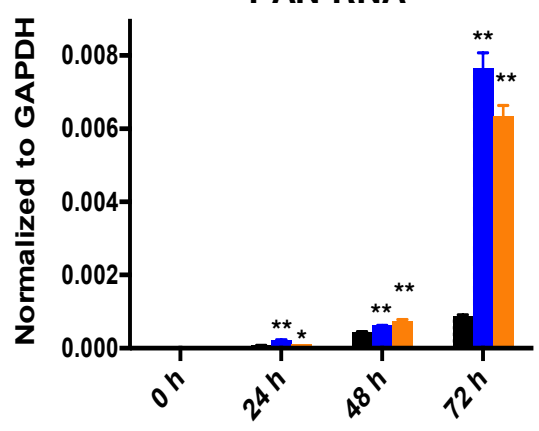

i
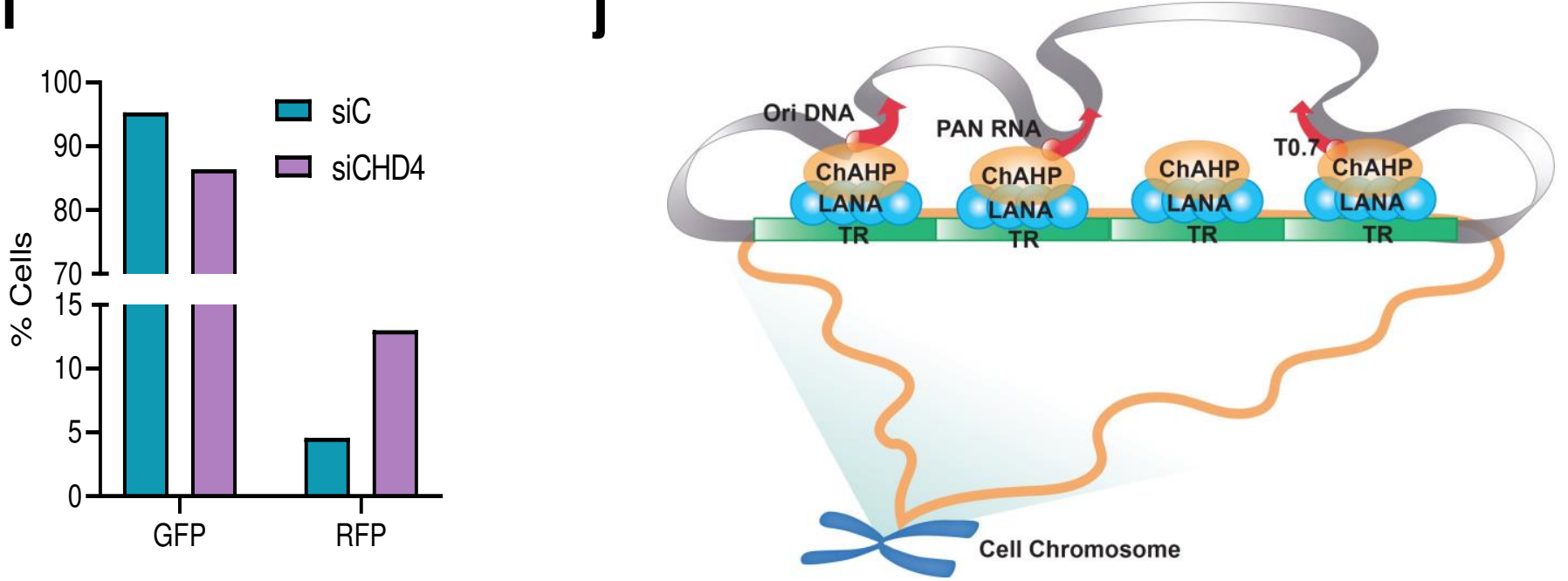

Fig. 6 
Figures
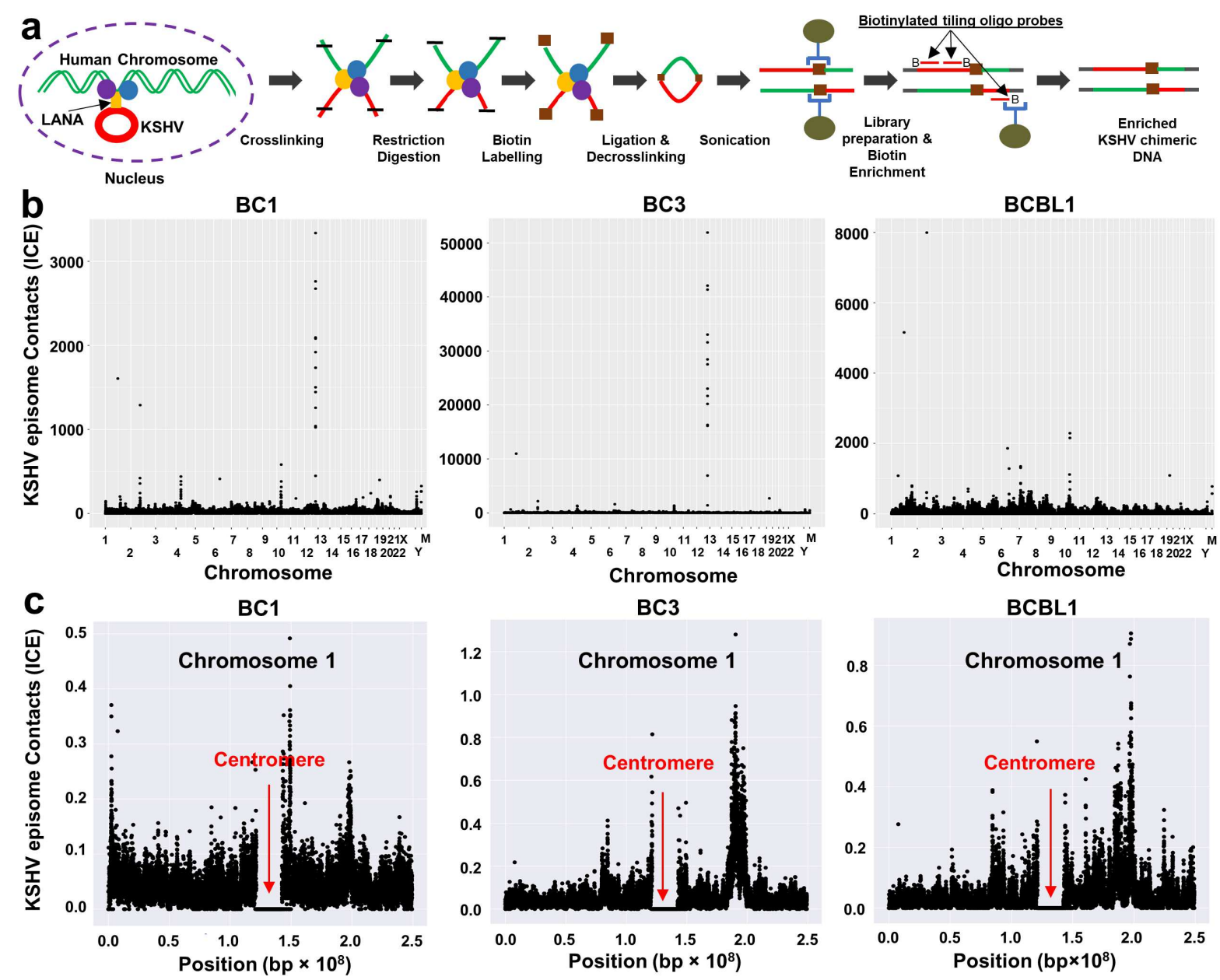

BC3
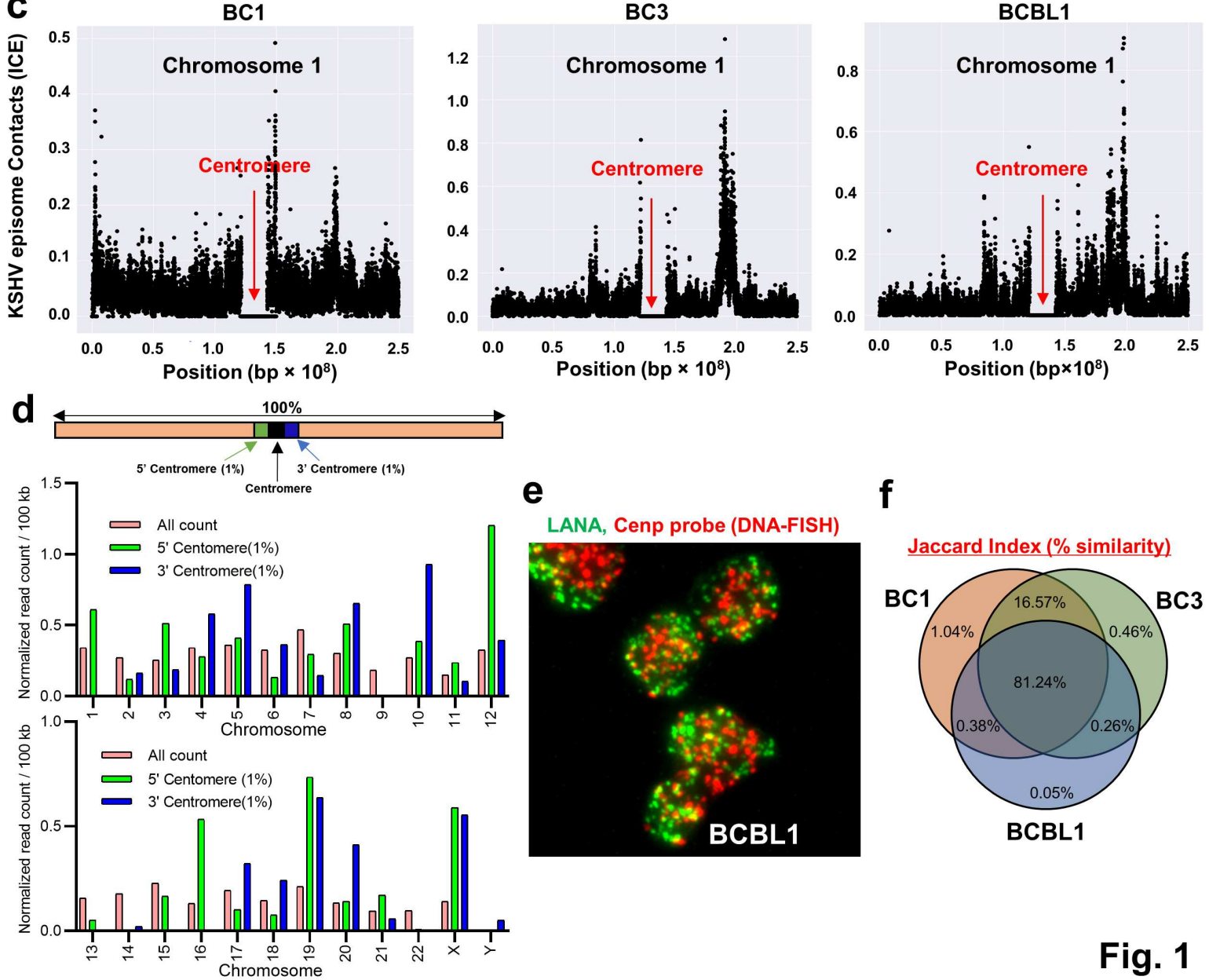

e

LANA, Cenp probe (DNA-FISH)

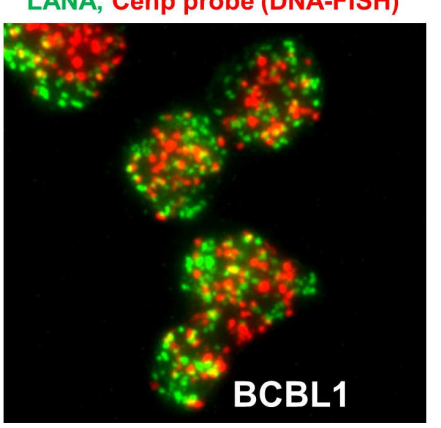

f

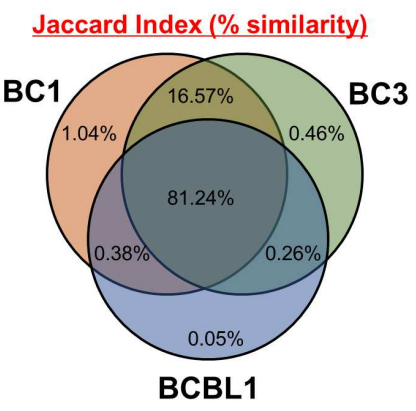

Fig. 1

\section{Figure 1}

Fig. 1 KSHV episome-tethering sites in KSHV positive cell lines. (a) Schematic workflow for Capture-HiC (CHi-C). (b) KSHV episome-docking sites on host cell chromosomes. CHi-C chimeric DNA ligation products composed of sequences derived from the KSHV and human genomes were mapped in three 
naturally-infected PEL cells. For BCBL-1 cells, three biological replicates were performed with nearly identical results (similarity 0.95 ). $\mathrm{M}$, mitochondrial chromosome. ICE-corrected profiles depicting sums of filtered read counts binned at $10 \mathrm{~kb}$ resolution are shown. (c) Selected chromosome 1 in zoomed view. Chromosome 1 dot plots were depicted, which displays enrichment of sequence reads near the centromere. Red arrows indicate the position of the centromere. Extended panels for all other individual chromosome for three cell lines are presented in Supplementary Fig. 1a-c. (d) KSHV episomes preferentially localize near the centromere. KSHV episome contacts were calculated in BCBL-1 cells for sequence reads in $1 \%$ (length of the respective chromosome) of the $5^{\prime}$-and 3 '- centromere and compared with the average of the total chromosome read count. (e) LANA protein locates near the centromere. DNAFISH was used to visualize the location of the centromere (red) and KSHV episomes were indirectly visualized by staining LANA dots (Green) in BCBL-1 cells. The centromere and LANA were probed using a Cenp probe and anti-LANA antibody respectively with IFA. The two signals were frequently co-localized, and 3D view and their image analyses are presented in Supplementary Fig. 2. (f) Jaccard statistics and Venn diagram. Venn diagram shows percent similarity of KSHV episome-tethering positions among BC-1, BC-3 and BCBL-1 cells. 


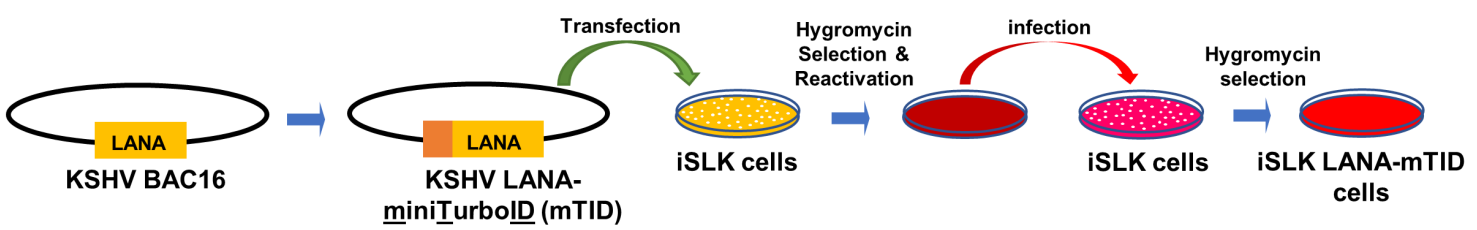

b

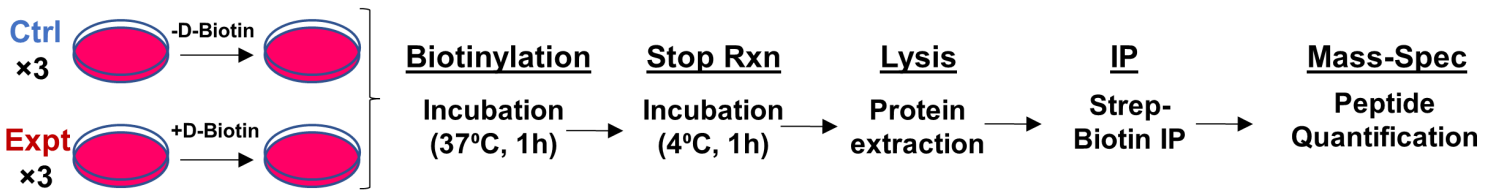

C

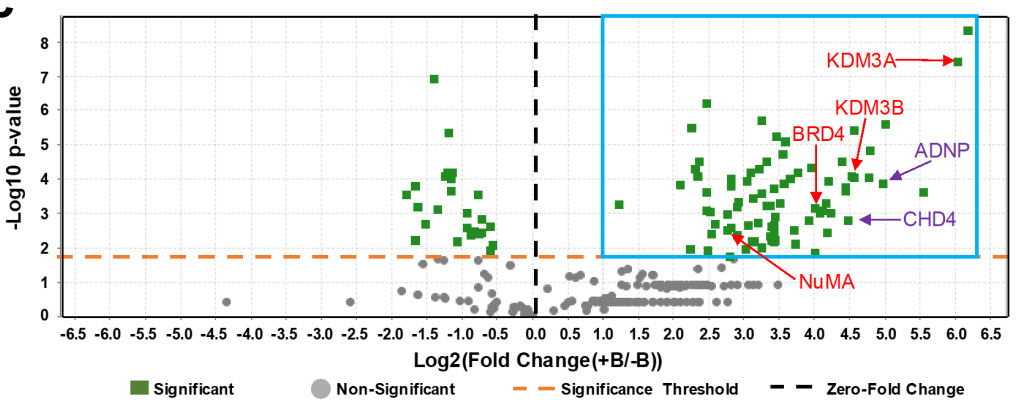

d
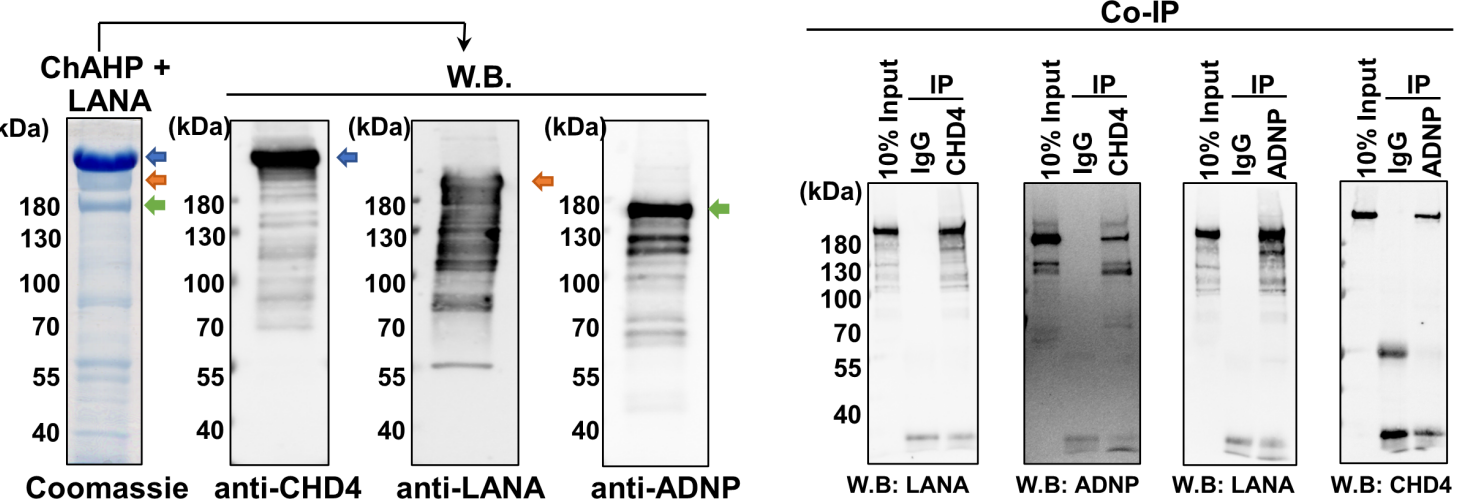

$\mathbf{e}$

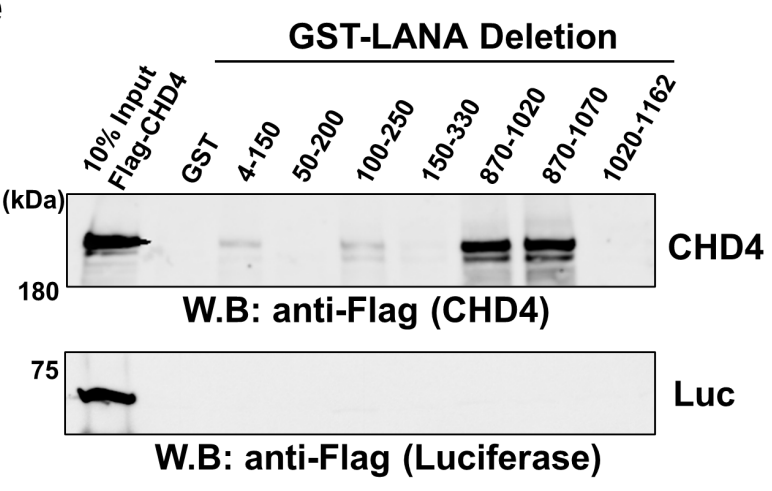

f
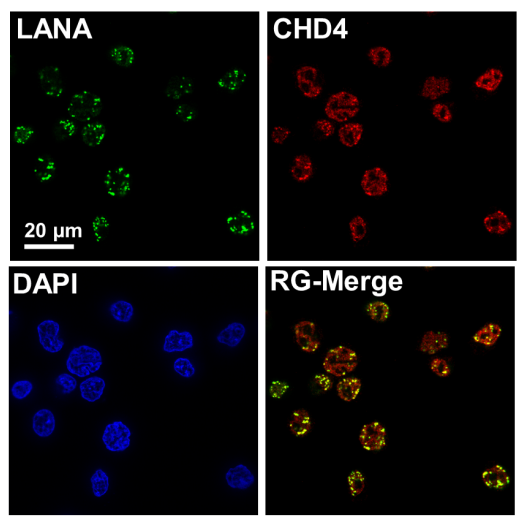

Fig. 2

\section{Figure 2}

LANA interacts with the ChAHP complex. (a) A schematic diagram of preparation of recombinant KSHV infected iSLK cells. A mini-TurboID (mTID) was fused to the N-terminus of the LANA open reading frame to generate KSHV LANA-mTID. KSHV LANA-mTID was transfected into iSLK cells followed by production of viral particles by stimulating cells with doxycycline $(1 \mu \mathrm{g} / \mathrm{ml})$ and sodium butyrate $(3 \mathrm{mM})$ for 5 days. Virus was used to infect iSLK cells and cells were selected with hygromycin $(1 \mathrm{mg} / \mathrm{ml})$ to obtain an iSLK- 
LANA mTID stable cell line. (b) Experimental design for preparing samples for protein ID. iSLK-LANA mTID cells were left un-incubated (-) or incubated (+) with D-Biotin $(500 \mu \mathrm{M})$ for $1 \mathrm{hr}$. Unincubated (-) cells were used as control samples. Each protein ID was performed in three biological replicates. (c) Volcano plot depicting proteins in close proximity to LANA. The volcano plot represents proteins identified in close proximity to LANA. Proteins with an abundance Log2 FC of greater than or equal to 1 and p-value less than 0.05 were selected and are shown by blue box. T-test was used for calculating the p-value. Purple color; ChAHP components. Red color; previously identified proteins as LANA interacting proteins. (d) LANA interacts with CHD4 and ADNP in vitro. LANA, CHD4 and ADNP complex, which consists of FlagLANA (gold arrow), Flag-CHD4 (blue arrow), and His-ADNP (green arrow) was prepared by co-infected three recombinant baculoviruses, and the protein complex was isolated in the presence of $500 \mathrm{mM} \mathrm{NaCl}$ and $10 \%$ glycerol with affinity purification. The authenticity of respective protein band was confirmed by immunoblotting with specific antibodies. Coomassie staining is shown. An equimolar amount (100 nM) of the three proteins were incubated in binding buffer and immunoprecipitated with the specific antibodies of one another. Ten percent of the reaction before immunoprecipitation was shown as controls. W.B: Western Blotting, Co-IP: Co-immunoprecipitation. (e) CHD4 interaction site on LANA. Purified GST-tagged LANA deletions, Flag-tagged luciferase and 41 Flag-tagged CHD4 were prepared from recombinant baculovirus infected Sf9 cells. An equal amount $(1 \mu \mathrm{g})$ of each LANA deletion protein was incubated with full length Flag-tagged luciferase $(1 \mu \mathrm{g})$ or Flag-tagged CHD4 $(1 \mu \mathrm{g})$ in binding buffer and interaction was probed with anti-Flag antibody. LANA deletion proteins used for this GST-pull down assay are presented in 910 Supplementary Fig. 4. (f) LANA co-localizes with CHD4 in BCBL-1. CHD4 and LANA were 911 probed with anti-CHD4 rabbit monoclonal antibody and anti-LANA rat monoclonal antibody, 912 respectively. Images were taken with Keyence fluorescence microscopy. Scale bar $(20 \mu \mathrm{m})$ is 913 shown 
a

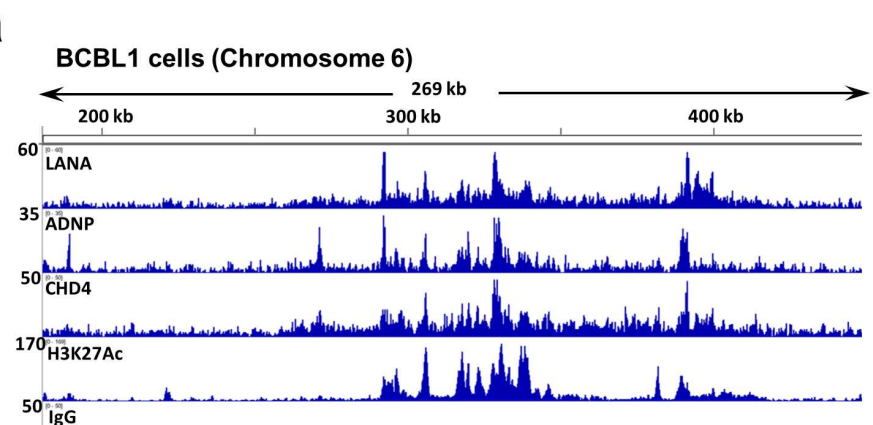

b

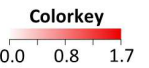

Colorkey

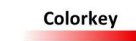

$0.4 \quad 0.7$

\begin{tabular}{l|l|l}
0.0 & 1.0 & 2.0
\end{tabular}

LANA CUT\&RUN LANA CUT\&RUN LANA CUT\&RUN
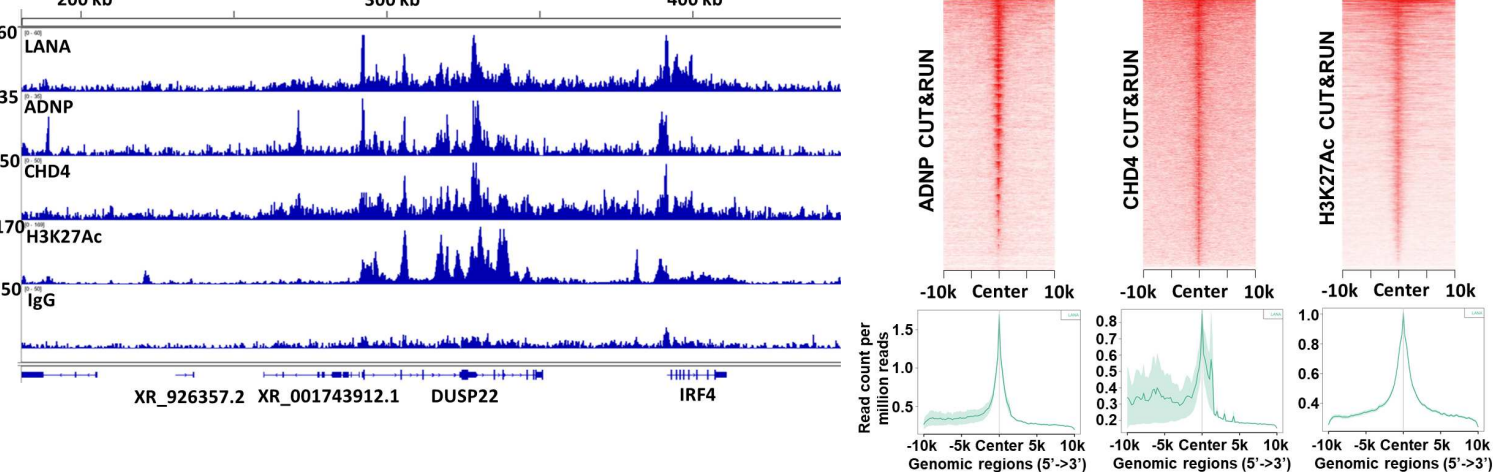

C

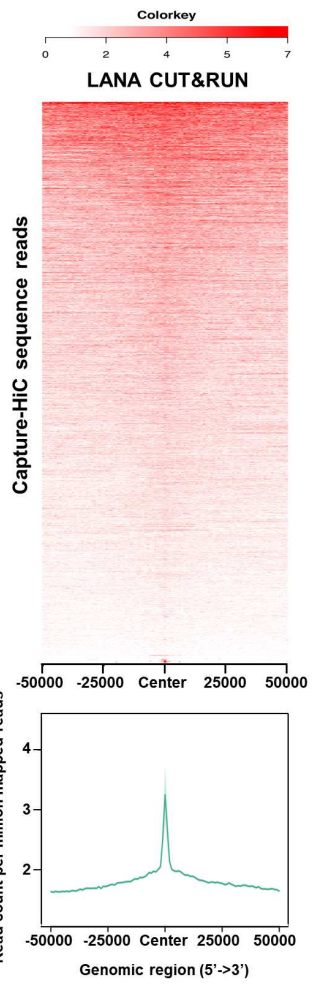

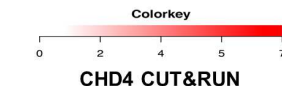
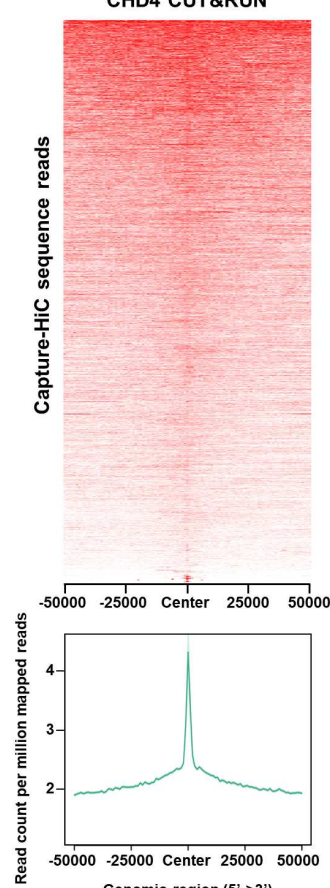

Genomic region $\left(5^{\prime} \rightarrow 3^{\prime}\right)$
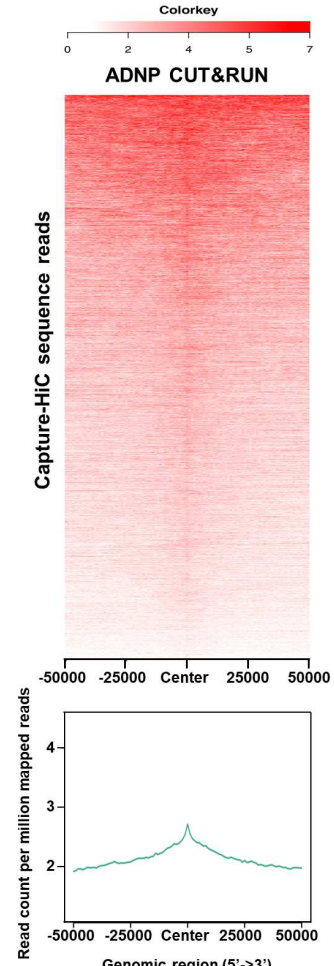

Genomic region $\left(5^{\prime}>3^{\prime}\right)$

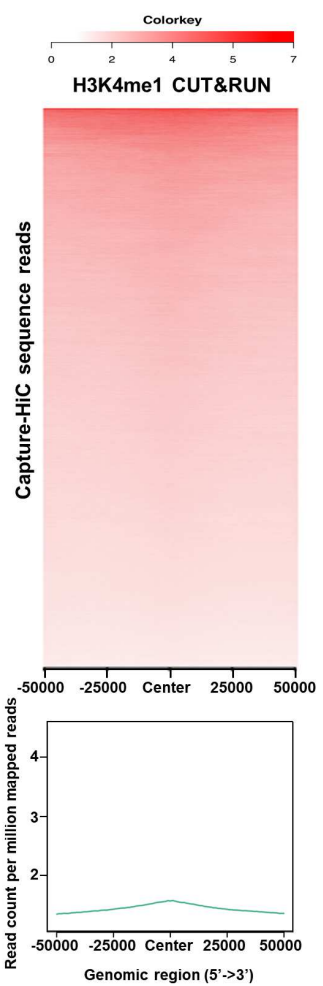

Genomic region (5'>>3')

d
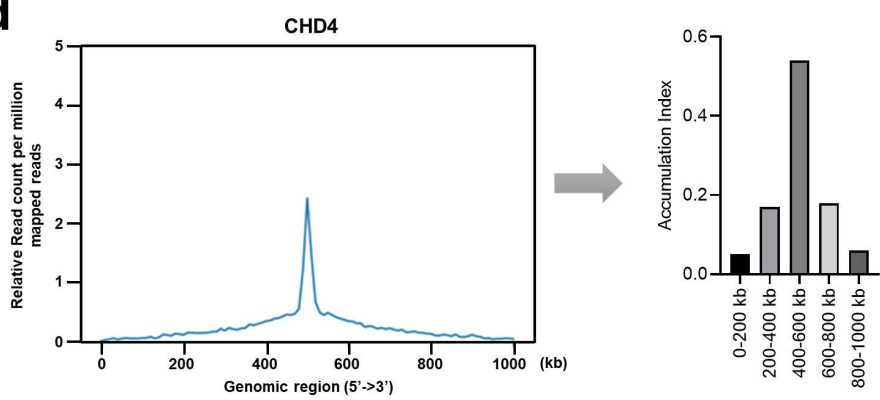

Fig. 3

\section{Figure 3}

Association of ChAHP complex binding with KSHV episome-tethering sites. (a) CUT\&RUN analysis for LANA, ADNP, CHD4 and H3K27Ac. The indicated antibodies were used for CUT\&RUN in BCBL-1 cells. CUT\&RUN peaks were visualized with Integrative Genomics Viewer (IGV) and a snapshot of one of the major binding sites (IRF4 super enhancer region) is shown. The number on the left-hand side denotes the height of the peak (e.g., read depth) and the number along the top denotes the positions on chromosome 
6. (b) Heatmaps for co-occupancies of LANA with ADNP, CHD4 and H3K27Ac on chromosomes. Heatmap (middle) and average profile (bottom) showing correlation of LANA enrichment (by color intensity and region) with ADNP, CHD4, and H3K27Ac occupancies. Average profile plot summarizing the heatmap (bottom). The lighter green shade represents the standard error (SEM) on the average profile plot. Color keys are shown on the each heatmap (top). (c) Heatmaps for correlation of CHi-C with CUT\&RUN signals. The association between $\mathrm{CHi}-\mathrm{C}$ chimeric reads and LANA, CHD4, ADNP and H3K4me1 CUT\&RUN peaks were depicted as heatmap and average profile plot. (d) Accumulation index around CHD4 binding sites. The accumulation 42 of CHD4 is displayed as a proportion of area under the curve of the relative average profile. Association between distance from CHD4 summit peak and relative chimeric sequence counts is shown as a bar chart. 


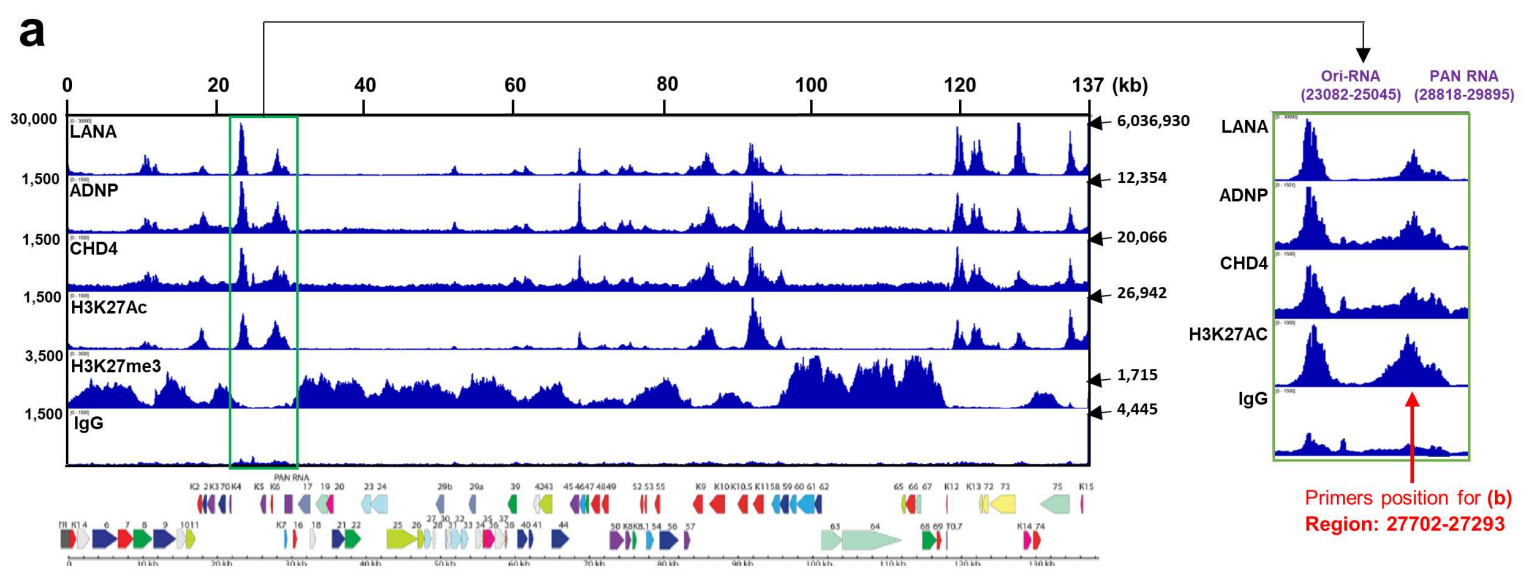

b

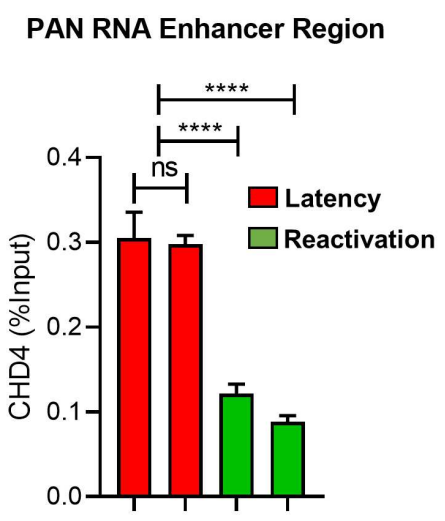

C
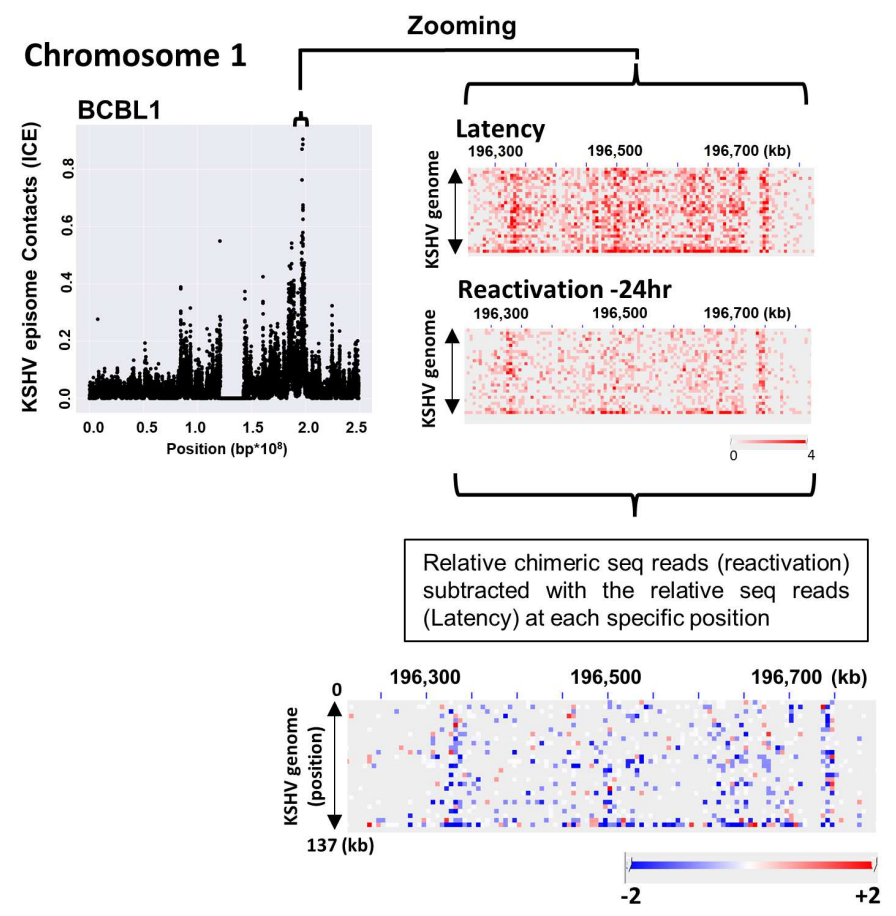

Fig. 4

\section{Figure 4}

LANA, CHD4 and ADNP colocalize on the KSHV genome. (a) CUT\&RUN analysis. The indicated antibodies were used for CUT\&RUN in BCBL-1 cells and sequence reads were mapped to the KSHV genome. An IGV snapshot and KSHV genome map are shown. The number on the left-hand side denotes the height of the peak (e.g., read depth), the number along the top denotes the position on KSHV genome in kilobase (kb) and the number on the right-hand side indicated by arrow denote the height of the peak on terminal 
repeat region (TR). Selected region is zoomed in to show Ori-RNA and PAN RNA region. Primer used in Fig. $4 \mathrm{~b}$ is also shown with red arrows and number show the exact location of the amplified region by the primers. (b) Regulation of CHD4 on the KSHV episome during reactivation. TREx-BCBL-1 cells were reactivated with doxycycline $(1 \mathrm{\mu g} / \mathrm{ml})$ and sodium butyrate $(3 \mathrm{mM})$ for $24 \mathrm{hrs}$. CUT\&RUN was performed on un-reactivated (latency) and reactivated cells for CHD4. CHD4 binding on the PAN RNA promoter region (red arrow in Fig. 4a, right panel) was calculated relative to the input sample ( $n=3$, two biological replicates are shown). (c) Detachment of KSHV episomes by reactivation. BCBL-1 cells were reactivated with doxycycline $(1 \mu \mathrm{g} / \mathrm{ml})$ and TPA $(20 \mathrm{nM})$ for $24 \mathrm{hrs}$. Chimeric sequence reads for un-reactivated (latency) and reactivated cells were mapped to the host chromosomes and visualized by Juicebox. Relative KSHV episome tethering during reactivation for the selected region on chromosome 1 was calculated by subtracting relative chimeric sequence reads in latency from relative chimeric sequence reads during reactivation. Blue and red dots indicate decreased and increased chimeric sequence reads respectively. 
a

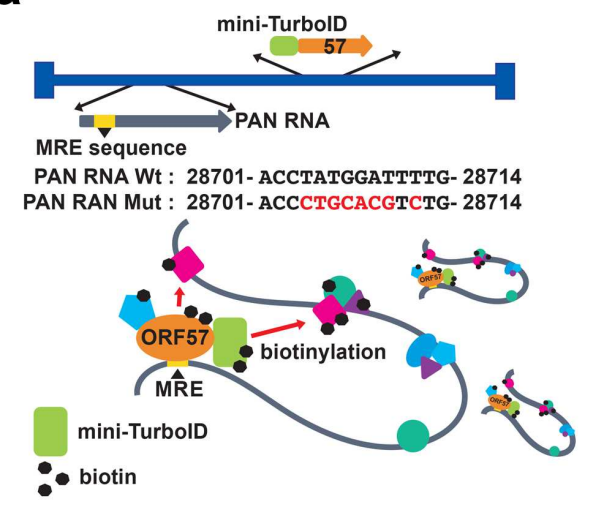

b
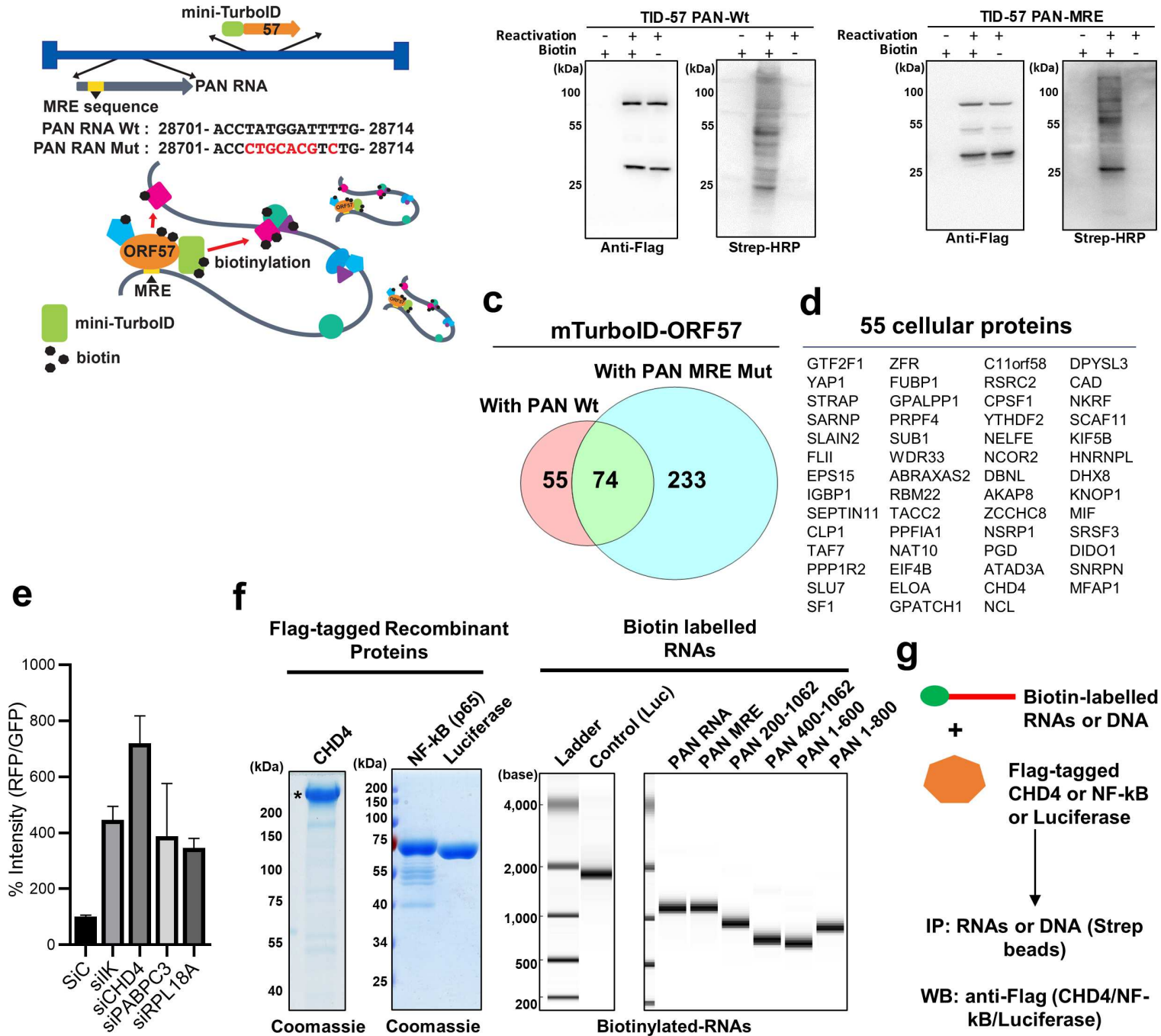

f

Flag-tagged Recombinant Proteins

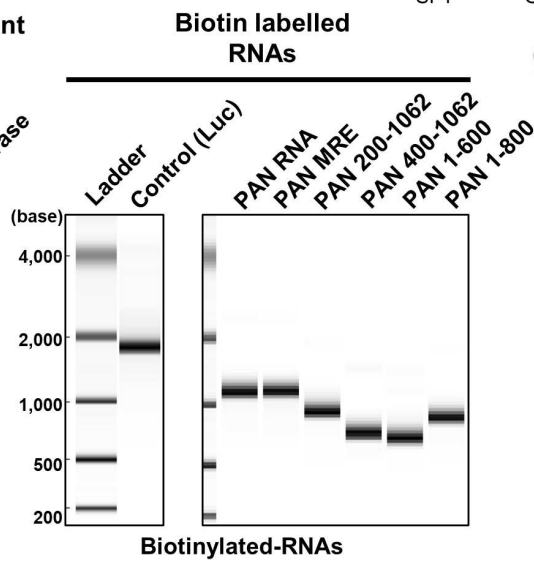

g
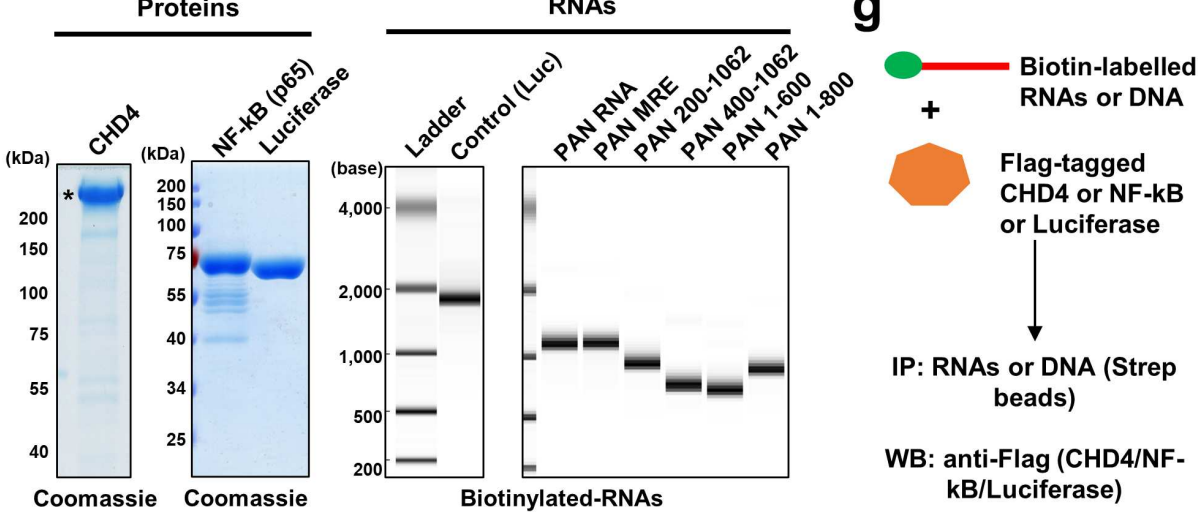

IP: RNAs or DNA (Strep beads)

WB: anti-Flag (CHD4/NF$k B /$ Luciferase)
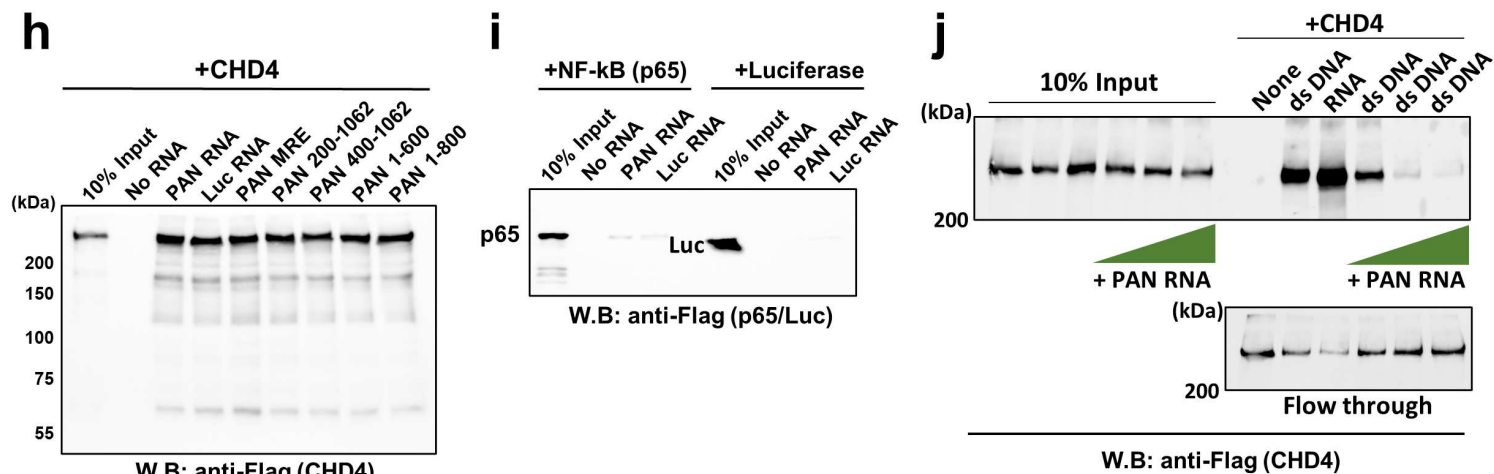

W.B: anti-Flag (CHD4)

Fig. 5

\section{Figure 5}

CHD4 is both an RNA and DNA binding protein. (a) A schematic diagram ofrecombinant KSHVs. MiniTurbolD is fused to the N-terminus of ORF57 open reading frame and the ORF57 binding site (MRE) of PAN RNA was mutated as shown. (b) Immunoblotting. Recombinant KSHV infected iSLK cells (TID-57 PAN-Wt and -MRE) were either untreated or induced for reactivation with doxycycline $(1 \mu \mathrm{g} / \mathrm{ml})$ and TPA $(20 \mathrm{nM})$ for $24 \mathrm{hrs}$. Blots were probed with either anti-Flag antibody (for mini-TurbolD-ORF57) or 
Streptavidin-HRP (for biotinylated proteins). (c) Venn diagram. The Venn diagram indicates the number of identified proteins with a $P$ value less than 0.05 . Each protein ID was performed in three biological replicates. (d) PAN RNA-mediated interacting proteins with ORF57. The proteins found enriched in PAN RNA962 WT samples are shown. The entire list of proteins and peptide counts are presented in Supplementary Table 2. (e) siRNA screening with KSHV reactivation as readout. Recombinant KSHV reactivation, which encodes RFP under control of the PAN RNA promoter, was used to screen the effects of KSHV reactivation. RFP signal intensity was measured with Image $J$, and the GFP signal was used as internal controls. Three randomly selected fields in the middle of each well were quantified and the average intensity was plotted. (f) Recombinant proteins and synthesized PAN RNAs. Recombinant Flagtagged proteins were expressed with baculovirus and purified with Flag-agarose beads. Coomassie staining of Flag-CHD4, Flag-NF970 kB (p65), and Flag-luciferase used for pull-down studies are shown. (g) Schematic for in vitro interaction assay performed in Fig. 5 (h-j). (h) CHD4 binds RNAs in a sequence972 independent manner. RNA pull-down was performed with the indicated biotinylated PAN RNA deletions, mutation (MRE), and irrelevant RNA (luciferase mRNA), and interaction was probed by immunoblotting with anti-Flag antibody. Beads alone (No RNA) was used for background 44 control. (i) RNA pull down was performed with indicated biotinylated RNAs, and p65 and Luciferase (Luc) were visualized by using anti-Flag antibody. (j) PAN RNA competes with CHD4 DNA binding. Pull-down analyses with biotinylated ssRNA or dsDNA was performed. CHD4 (100 nM) was incubated with biotinylated RNA (100 nM) or biotinylated dsDNA (100 nM) in $40 \mu \mathrm{L}$ binding buffer. Increasing amounts of non-biotinylated PAN RNA at 1:1, 1:10, and 1:20 (dsDNA vs. ssRNA) were also incubated, and precipitated CHD4 protein in the pull-down was probed with anti-Flag antibody. Flow-through and $10 \%$ of the input reaction before pull-down were used as control. 
a

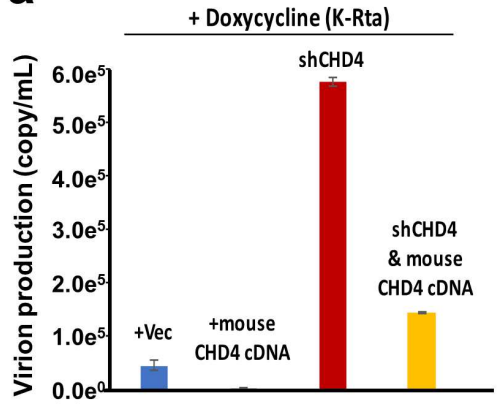

d

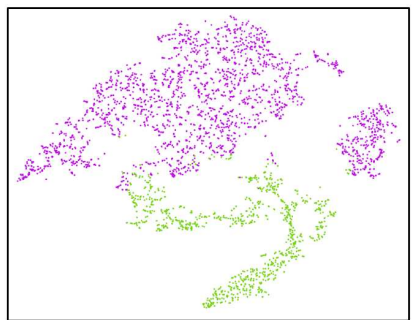

KSHV expression (-)

KSHV expression (+) b

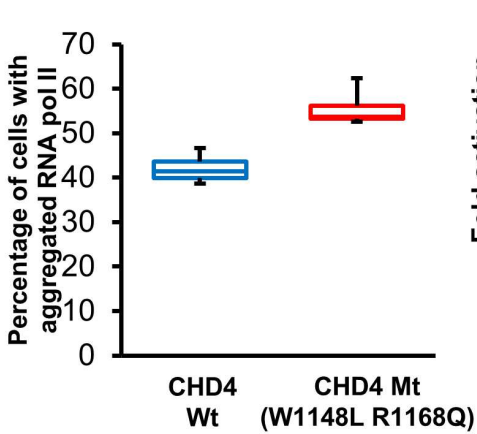

C

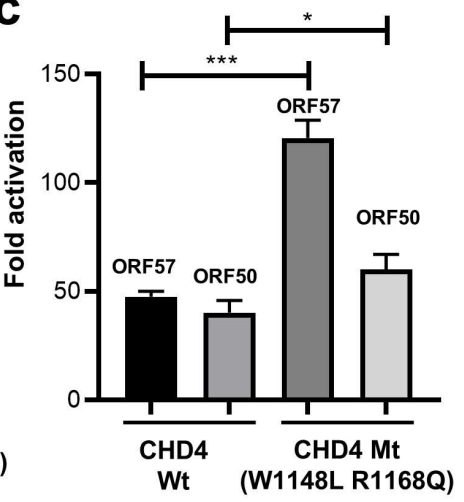

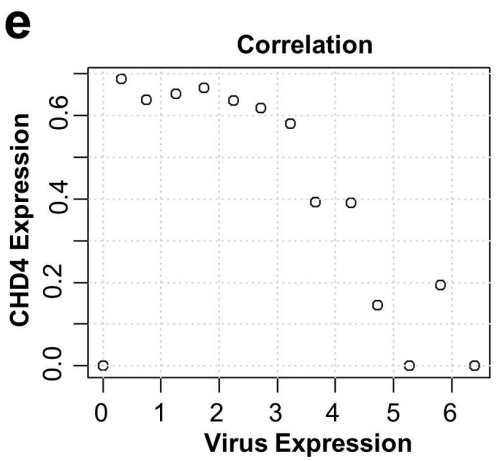

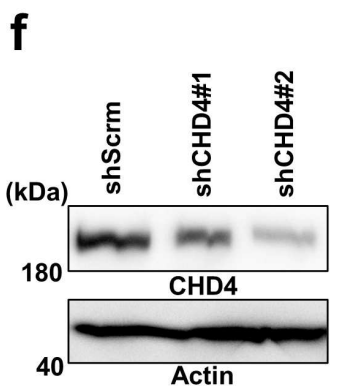

g $\mathrm{CHD} 4 \quad \mathbf{h}$
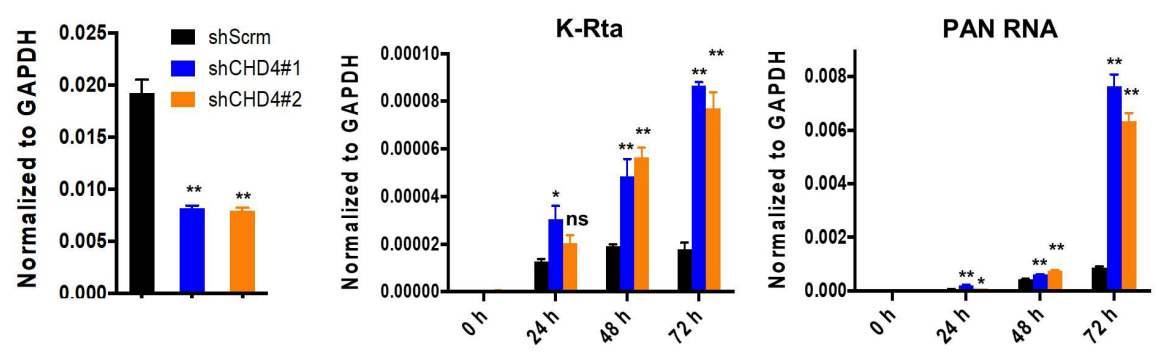

i

j
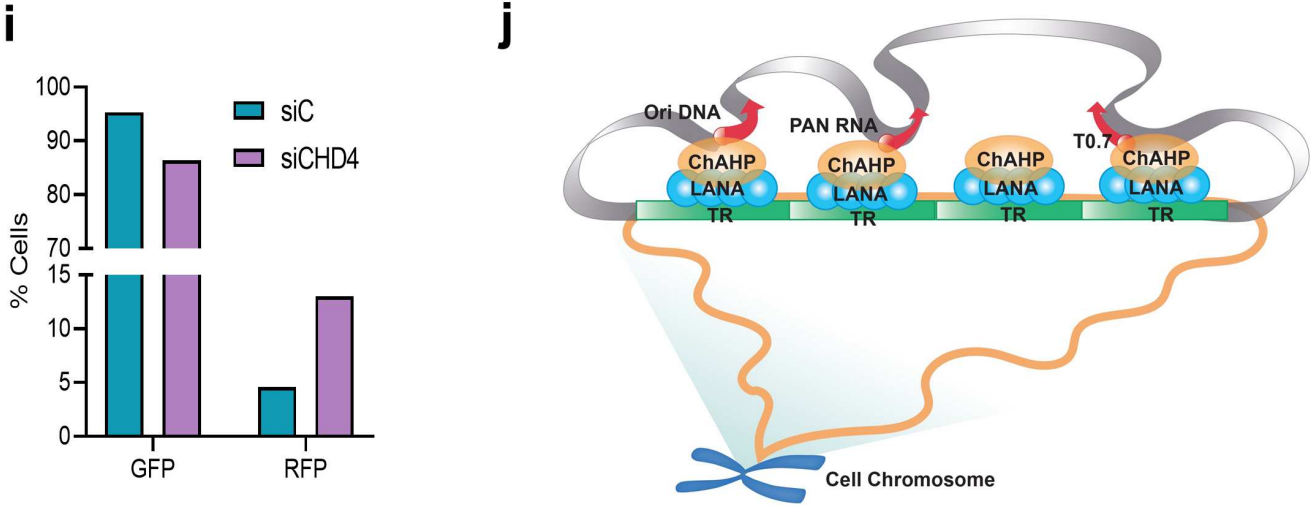

Fig. 6

\section{Figure 6}

CHD4 is important for latency maintenance and establishment. (a) CHD4 overexpression, knockdown and complementation. The iSLK.219 cell line was transfected with empty vector (Vec) or mouse CHD4 cDNA or human CHD4 shRNA, or CHD4 shRNA and mouse CHD4 cDNA for 48 hrs followed by KSHV reactivation with doxycycline $(1 \mu \mathrm{g} / \mathrm{ml})$ for 5 days. Encapsidated KSHV genomes in the culture supernatants were measured by qPCR to assess effects of CHD4 overexpression or knockdown on KSHV 
reactivation. (b) CHD4 ATPase activity is important for prevention of nuclear aggregate formation. iSLK cells latently infected with BAC16-Wt was transfected with Flag-tagged mouse wild type CHD4, or an ATPase domain mutant was transfected and stained with anti-Flag. Transcriptional factory formation was assessed by staining RNAll. The percentage of aggregate formation in Flag-positive cells was measured and plotted. Three biological replicates were performed, and 50 cells were counted in each of the three biological replicates. (c) CHD4 ATPase activity is important for inhibiting viral gene expression during reactivation. iSLK.219 cells were transduced with an equal amount of CHD4 wild type (wt) and CHD 4 mutant (mt) lentivirus for 2 days. Cells were 45 reactivated with doxycycline $(1 \mu \mathrm{g} / \mathrm{ml})$ for 2 days and ORF50 and ORF57 gene expression was quantified by RT-qPCR. Untreated cells were used for calculating the relative expression of ORF50 and ORF57. GAPDH was used as a negative control. ${ }^{*} \mathrm{p}<0.05$, $\star \star \star x p 0.001$ (comparing with CHD4 Wt). (d-e) Single cell sequencing. iSLK.219 cells were reactivated by induction of K-Rta expression from doxycycline inducible promoter for $24 \mathrm{hrs}$. Single-cell sequencing was performed with the $10 x$ Genomics platform. A cell suspension was prepared followed by cell partitioning and 3' cDNA synthesis on the Chromium Controller and dual-index library preparation with 10x Genomics kitted reagents. (d) tSNE. tSNE was applied to separate cell clusters and KSHV gene (d, left panel) and CHD4 (d, right panel) expressing cells were marked. (e) Negative correlation between CHD4 and viral gene expression. Cells were divided into groups based on the amount of KSHV transcripts. A table and distribution of cell numbers and amount of KSHV transcripts are shown in Supplementary Fig. 6a-b. Intervals used to separate cell groups are also listed in Supplementary Fig. 6b. A correlation was established based on CHD4 expression in different groups of KSHV transcripts. (f-h) CHD4 knockdown and KSHV lytic gene expression during de novo infection. 293T cells were transduced with scramble shRNA (shScrm) or shCHD4 and stably selected with antibiotic. Immunoblot (f) and RT-qPCR (g) analysis of CHD4 in the 293T stable cell lines are shown. 293T stable cells were infected with rKSHV.219 for 24 hrs and total RNA was harvested at the indicated time points. (h) K-Rta and PAN RNA, was measured by RT-qPCR and normalized to GAPDH. ${ }^{*} p<0.05,{ }^{*} p<00.01$ (comparing with shScrm). (i) Flow cytometry. 293T cells were transfected with control siRNA (siC) or CHD4 siRNA (siCHD4) for 48 hrs followed by infection with rKSHV.219 virus for 96 hrs. Flow cytometry analysis was performed to calculate the number of GFP- and RFP-positive cells in siC and siCHD4 knockdown cells. (j) KSHV latency model with ChAHP. KSHV episomes 46 tether at epigenetically active regions with the ChAHP complex, and TRloaded LANA/ChAHP prevents robust viral IncRNA expression. The action of robust IncRNA expression in lytic KSHV infection functions as an enhancer for viral ORFs that are physically neighboring with IncRNA genomic regions. Stimulation of K-Rta, in part by decreased CHD4 expression, triggers robust PAN RNA expression in the presence of ORF57, which competes with CHD4 DNA binding to enhance KSHV reactivation and detach $\mathrm{KSHV}$ episomes from CHD4-enriched host chromosome regions.

\section{Supplementary Files}

This is a list of supplementary files associated with this preprint. Click to download.

- SupplementaryFigures.pdf 
- SupplementaryTable1.xls

- SupplementaryTable2.xls

- SupplementaryTable3.xls 